\title{
Chapter 5 \\ Extended Non-destructive Testing Technique Demonstration in a Realistic Environment with Technology Assessment
}

\author{
Romain Ecault, Ana Reguero Simon, Célian Cherrier, Paweł H. Malinowski, \\ Tomasz Wandowski, Mirosław Sawczak, Kai Brune, Hauke Brüning, \\ Mareike Schlag, Johannes Derksen, Welchy Leite Cavalcanti, \\ Laurent Berthe, Maxime Sagnard, Wiesław M. Ostachowicz, \\ Saverio De Vito, Andreas Helwig, Rainer Stössel, Damien Segur, \\ Apostolos Chamos, and Konstantinos Tserpes
}

\begin{abstract}
This chapter highlights two advances towards a higher maturity of versatile extended non-destructive testing (ENDT) procedures. Full-scale demonstration tests are presented in realistic user application cases that involve typical production or repair scenarios. Subsequently, the investigations used to assess the probability
\end{abstract}

R. Ecault ( $\varangle)$

Airbus Operations S.A.S., 316, route de Bayonne, B.P. D4101, 31060 Cedex 9 Toulouse, France e-mail: romain.ecault@airbus.com

A. Reguero Simon

Aernova Composites Illescas Sau, Parque Industrial y Tecnológico, Avda Barajas, 3 Illescas, 45200 Toledo, Spain

C. Cherrier

Automation W+R GmbH, Messerschmittstraße 7, 80922 Munich, Germany

P. H. Malinowski · T. Wandowski · M. Sawczak · W. M. Ostachowicz

Institute of Fluid-Flow Machinery, Polish Academy of Sciences, Fiszera 14, 80-231 Gdańsk,

Poland

K. Brune $\cdot$ H. Brüning $\cdot$ M. Schlag $\cdot$ J. Derksen $\cdot$ W. Leite Cavalcanti

Fraunhofer Institute for Manufacturing Technology and Advanced Materials IFAM, Wiener Str.

12, 28359 Bremen, Germany

L. Berthe $\cdot$ M. Sagnard

PIMM, CNRS-ENSAM ParisTech, 151 Bd de l'Hôpital, 75013 Paris, France

S. De Vito

ENEA - National Agency for New Technologies, Energy and Sustainable Economic

Development, TERIN-FSD, Portici Research Center, P.le E. Fermi 1, 80055 Portici, Italy

A. Helwig · R. Stössel

Airbus Defence and Space GMBH, Willy-Messerschmitt-Straße 1, 82024 Taufkirchen, Germany

D. Segur

CEA LIST, CEA Saclay, 91191 Gif-sur-Yvette, France

(C) The Author(s) 2021

W. Leite Cavalcanti et al. (eds.), Adhesive Bonding of Aircraft Composite Structures, https://doi.org/10.1007/978-3-319-92810-4_5 
of detection (POD) are detailed for the respective ENDT processes and applicationrelevant scenarios in a realistic environment. Although some results indicated that some additional in-depth investigations would be even more enlightening, these demonstrations still clearly showed that developments and progress described in the previous chapters have enabled some of the technologies to achieve a maturity that is sufficient to proceed towards industrial implementation. Some ENDT techniques revealed the presence of contaminants on real structural parts with unknown contaminant amounts. For the first time, POD results obtained for ENDT investigations are presented. Some ENDT procedures permitted POD results to be obtained for several scenarios, while others showed technologically relevant POD only for certain scenarios. For two ENDT techniques, determining the POD helped to enhance the respective testing and evaluation procedures. In most of the cases, it was possible to estimate a preliminary quantification of POD by giving the POD90/95. For some techniques, this value was below the lowest contamination degree.

Keywords Full-scale demonstration tests - Realistic scenarios • CFRP repair • Probability of detection (POD) $\cdot$ Bonding assessment

\subsection{Introduction to the Full-Scale Demonstration Event}

In the previous chapters, after providing an introduction to extended non-destructive testing (ENDT) and quality assessment (QA) in adhesive bonding processes relevant to the manufacture or repair of composite structures, we detailed the advances achieved so far, e.g., in the European joint research projects ENCOMB and ComBoNDT. We highlighted substantial technical progress under the framework of laboratory-scale investigations and inferred that it promises to facilitate the implementation of procedures to reach a technology readiness level (TRL) enabling their application in field-level sensing systems within industrial adhesive bonding processes used for the manufacture or repair of joints, including those based on CFRP adherends.

In this chapter, our objective was to test each EDNT technique in a real-world environment-outside the laboratory - and on realistic parts. Within the framework of the ComBoNDT project, this final test was used to assess the technological maturity with regard to aeronautical applications. It is a demonstration considered to be as close as possible to real industrial processes, and we consider the results highly representative.

To this end, as part of the ComBoNDT project [1], we organized a "Demonstration Days" week in Bremen at the Fraunhofer IFAM site, which offered a dedicated

\footnotetext{
A. Chamos

EASN Technology Innovation Services, Terweidenstraat 28, 3440 Budingen, Belgium

K. Tserpes

Laboratory of Technology \& Strength of Materials, Department of Mechanical Engineering \& Aeronautics, University of Patras, 26500 Patras, Greece
} 
environment. Using a teamwork approach, the partners transported their individual mobile setups to Northern Germany, thereby moving them out of their base laboratory. The Fraunhofer IFAM site was a suitable location for the demonstration because it provided each partner with logistical and automatization capabilities. The available equipment included facilities for the preparation of industrially relevant bonded composite parts, which involved the intentional application of contaminants-an obligatory step in the bonding process of the demonstration.

The demonstration consisted of a set of process steps performed by different actors in a coordinated manner that was designed to be as linear as possible. Our basic idea was to have-in the same place and at the same time-all the steps of a technologically relevant bonding process, either in production or in repair, that closely followed industrial processes. To give our readers a rough outline of the complex procedure, we ensured that the assessed panels were first prepared for bonding using an appropriate cleaning process before being partially contaminated. Then, we applied the first batch of ENDT tools to perform the steps of the monitoring process, specifically those dedicated to adherend surface quality assessment. Afterwards, we conducted the adhesive bonding operations and performed post-bonding characterization using the second batch of ENDT techniques. We would like to highlight that these full-scale tests also acted as a blind test or round-robin test because the applied contamination degree was neither checked using laboratory-based reference analysis nor communicated to the inspection specialists. This holds true for both the production and repair panels assessed here.

In this chapter, we describe the performed full-scale tests. First, we present the parts produced or recovered for this demonstration. Then, we describe the preparation of the respective panels for testing, whereby the panels for the production user case and the selected repair user case are addressed separately. In the latter case, the performed scarfing is described in addition to the contamination preparation, while the respective bonding process is presented for both applications. The ENDT results are then given for each panel, starting with the surface quality control and following this with the bonding assessment. Thus, the chapter is organized following a real implementation scenario, as explained in the previous paragraph. Finally, we present a summary of the full-scale demonstration.

Aiming for quantitative evaluation, we also give some initial elements on the probability of detection (POD) in this chapter. This method is used systematically in aeronautics to assess the detection capability of conventional NDT approaches. In this respect, we used CIVA software for some of the analytical ENDT data gathered at the coupon specimen level (described in more detail in Chaps. 3 and 4, respectively) to model the obtained POD curves.

Finally, a few words on project dissemination are given to conclude the chapter. 


\subsection{Setup of the Full-Scale Demonstration: Materials, Workflow, and Operations}

This section describes the planning and implementation of all the operations necessary to conduct the full-scale demonstration. The emphasis hereby is on the provision of the materials, the applied workflow, and the operational sequence. As previously highlighted, all three aspects were maintained as close as possible to a sequential industrial process. Almost all the ENDT techniques were available and employed onsite during the three-day demonstration period. In exceptional cases, further ENDT investigations were conducted elsewhere, whereby either all the panels or one specific panel were sent to the respective partner lab to perform the testing there.

\subsubsection{Providing Real and Realistic Parts}

In the following, we detail the geometry and composition of the realistic parts used for the production user case and the real parts employed for the repair user case within the ComBoNDT joint research project.

\subsubsection{Manufacturing of a Flat Panel for the Production User Case}

We designed a panel representing a wing application for the production user case with the intention that it should differ from the part used for the repaired fuselage application to highlight the versatility of the ENDT approaches. The manufacturing process of the panel was performed at Aernnova and comprised two different steps: the manufacture of the T-stringers and the manufacture of the skins. Hexcel M21E was used for the manufacture of all samples. Similar to the repair user case, we chose the dimensions to represent the correct order of magnitude of a tested region while providing enough space to test the application of several ENDT methods.

The stringers were manufactured following current industrial production guidelines. The web was shaped by joining two C-shape preforms that had been produced using automated tape laying (ATL). First, a flat panel was laminated and placed on a hot-forming tool to obtain the C-shape. After that, both C-shaped parts were joined. Finally, the element was trimmed to obtain the six T-stringers with the required dimensions. Four of these were dedicated to mechanical testing and two were kept for the full-scale demonstration. The stringers were inspected by ultrasonic C-scan inspections and no particular defects were revealed (Fig. 5.1).

The skin samples were manufactured with the following carbon fiber distribution Table 5.1, which was similar to the panels used for mechanical testing.

The production began with the manufacture of a $3500 \mathrm{~mm}$ by $1000 \mathrm{~mm}$ flat panel laminated using ATL. The distribution of the skin samples within this panel is depicted in Fig. 5.2. 


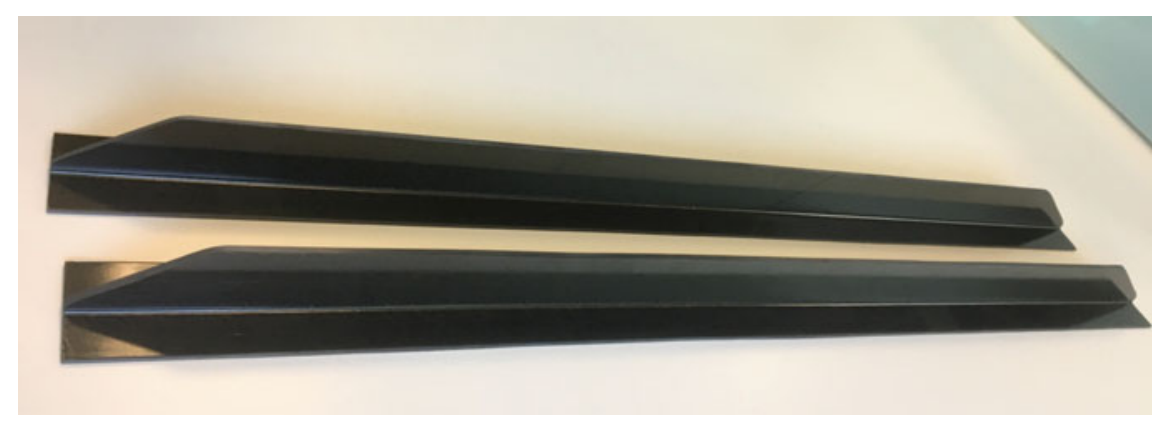

Fig. 5.1 Image of manufactured T-stringers

Table 5.1 Carbon fiber distribution of manufactured samples

\begin{tabular}{l|l|l|l}
\hline Part & Specimens & Plies & Stacking \\
\hline Skin & 4 & 20 & {$[ \pm 45 / 90 / 02 / 90 / \pm 45 / 0 / 90] S$} \\
\hline
\end{tabular}

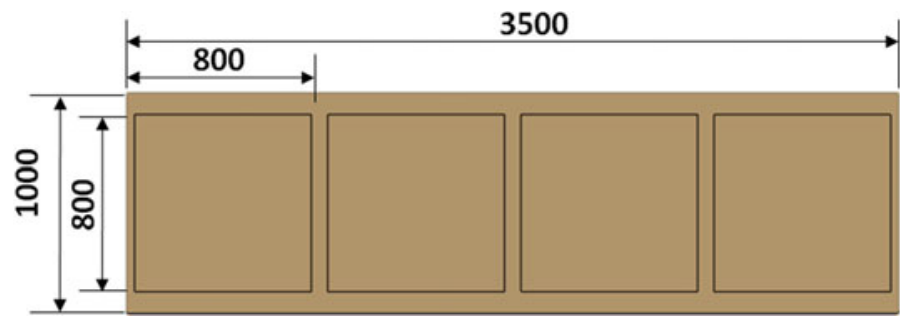

Fig. 5.2 Flat panel manufactured for four square skin samples; dimensions are given in $\mathrm{mm}$

After the curing cycle, the flat panel was automatically inspected using pulseecho equipment with water as the coupling medium to ensure the quality of the part manufactured. No defects were revealed (see Fig. 5.3). After the inspection, a trimming operation was performed to obtain the number of skin samples required for the mechanical testing (see Chap. 2) and full-scale demonstration.

The bonding operation of the stringer onto the composite skin was part of the fullscale demonstration workflow. Thus, intentional contamination needed to be done before bonding. It should be noted here that due to the dimensions of the bond line and the presence of two stringers, all the ENDT investigations could be tested using the same panel. More details are provided in the next section. 

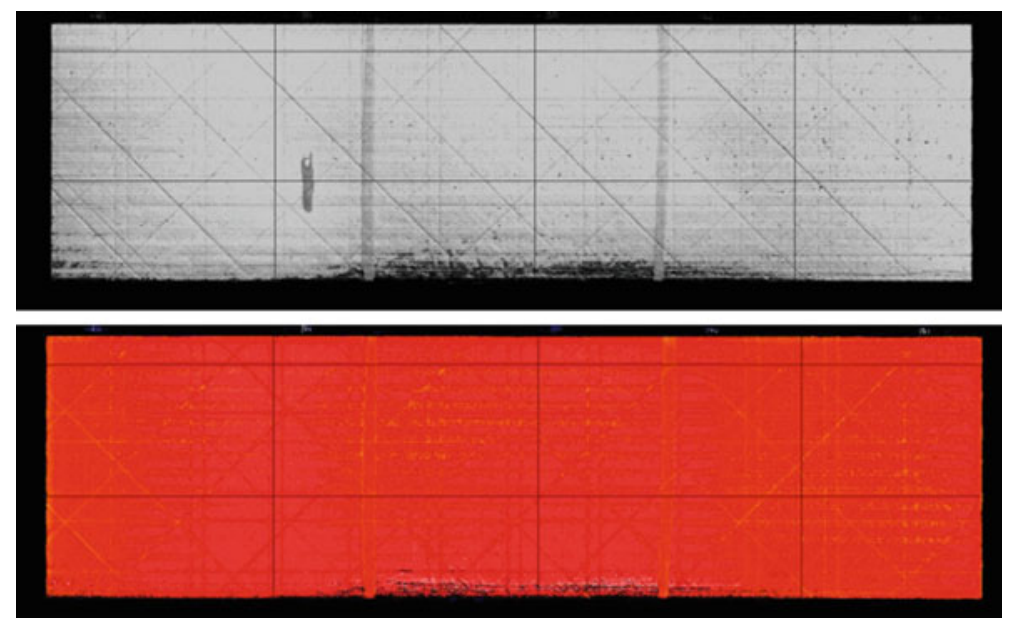

Fig. 5.3 Color-coded representation of the findings from the ultrasonic inspection of the flat panel

\subsubsection{Tear-Down Cell for the Representative In-service Repair User Case}

To demonstrate the ENDT techniques on full-scale specimens, we performed a bonded repair on a real part as we considered this to best represent a real application scenario, wherein the part being repaired has undergone a variety of loads and environmental conditions. For this purpose, fuselage panels were recovered from an A350 aircraft used by Airbus for full-scale static testing. These panels had been part of such a structural test, but had remained in good condition and could thus be employed in the demonstration (see Fig. 5.4). The size of each panel was approximately $1 \mathrm{~m}^{2}$, which was adequate for several reasons:

1. The panels were large enough to be considered full scale, and they were in the order of magnitude of what would be tested by ENDT with a significant TRL.

2. Their size enabled a sizeable bonded repair to be made with enough space to gather data to test several ENDT tools, with the investigation performed in both the contaminated and reference areas.

3. They provided enough free edges to calibrate the measurement setup if necessary and to grasp and hold the parts in order to manipulate them manually or using a robotic arm.

As explained within the workflow and operations section, we assessed three panels. One panel was used for "intrusive" ENDT, such as monitoring based on magnetostrictive sensors and electromechanical impedance, for which it was necessary to bond sensing devices to the part. As the setups required for surface quality measurements using laser-induced fluorescence spectroscopy (LIF) and vibrometry were not moved to the demonstration event location, one panel was kept at IMPPAN in Gdansk after scarfing and without bonded repair. Finally, one panel was 


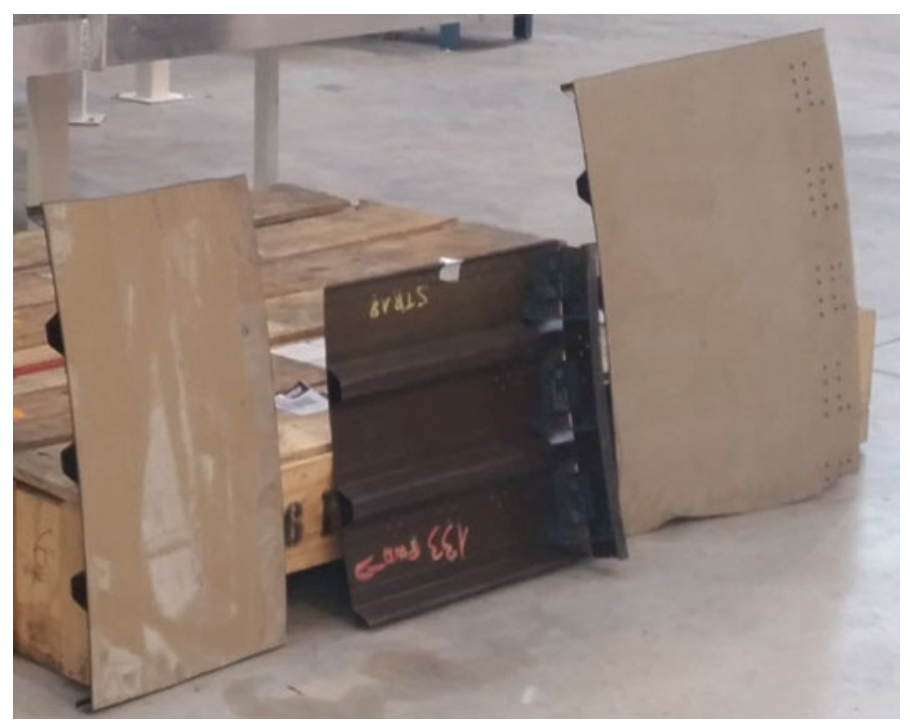

Fig. 5.4 A350 aircraft fuselage panels provided by Airbus for the demonstration of a full-scale bonded repair

used for the inspection using non-intrusive bonding assessment techniques, namely nonlinear ultrasound (NLUS) and laser shock adhesion testing (LASAT) (as outlined in the workflow and operations section).

As they were taken from the same section, these three panels were similar, but they were not identical in terms of geometry, number of stiffeners or thickness. A detailed view of two of the three panels is presented in Fig. 5.5. The skin thickness was in the

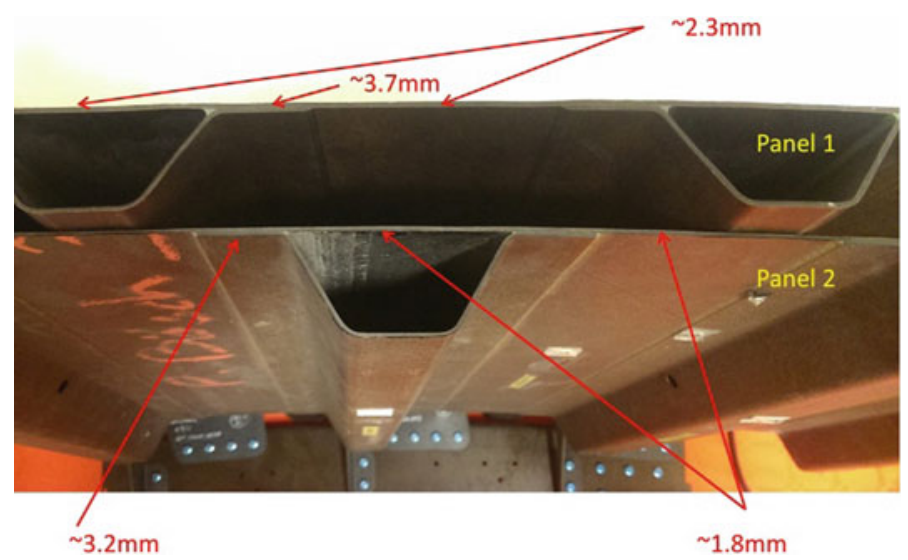

Fig. 5.5 Detailed view of the A350 fuselage panels used for the demonstration of quality assessment during bonded repair; the skin thickness is indicated at specific positions 
range of $1.5-2.5 \mathrm{~mm}$, which corresponds to the coupon and pilot sample geometries because-once repaired-the assembly thickness increased to about $2.5 \mathrm{~mm}$. In addition, either two or three omega stringers reinforced the skin. The stringer foot was about $1.5 \mathrm{~mm}$, which means that the total thickness was a maximum of $4 \mathrm{~mm}$ in this region. The panel material was the composite IMA/M21E (Hexcel), similar to the material used for the coupon and pilot samples.

The three panels were used to simulate a scarfed bonded repair. As the focus is on testing the adhesive bond between the scarfed skin and the repair patch, we decided to use these structures without introducing any damage to save time and costs. While it could have been subjected to, for example, delamination caused by a drop tower, this would have been removed by the subsequent scarfing operations anyway. Instead, we defined the dimensions of the bonded repair patch based on the standard for delamination that was not actually performed.

\subsubsection{Participants and Operations}

This section links the list of operations, the main steps, and the participants in the fullscale demonstration. All the information is compiled for each scenario in Table 5.2, outlining the main process steps, operations, the corresponding ComBoNDT project partners in charge, and the used techniques or procedures. It should be noted that the whole full-scale demonstration in the ComBoNDT joint research project was led by Airbus and Fraunhofer IFAM.

The following two sections detail how these operations were conducted during the full-scale demonstration event. Hereby, the focus is on the workflow, the contamination process, and the bonding operations executed within the framework of the production or repair user cases.

\subsection{Production User Case}

\subsubsection{Workflow Overview}

The operations within the production user case comprising distinct contamination scenarios are described in Fig. 5.6 following the ComBoNDT (with the name of the contributing partners) project. First, the skin and stringers for the full-scale demonstration were provided by Aernnova. The quality of the skin was checked at Aernnova using conventional ultrasound testing, as outlined in the previous section. In this case, one panel was sufficient for all the ENDT approaches. At the beginning of the three-day full-scale demonstration, the skin and stringer were contaminated separately with RA and FP formulation, successively. The skin was then tested using FTIR (Airbus, AGI), e-nose (AGI), and e-nose (ENEA) to conduct the 
Table 5.2 List of operations for production and repair user cases comprising scenarios with the intentional contamination of an adherend surface by a release agent (RA) and fingerprint (FP, with either sweat or hydraulic oil) or de-icing fluid (DI) as performed during the three-day onsite demonstration event, indicating the corresponding ComBoNDT project partners in charge. The involved ENDT techniques comprise the aerosol wetting test (AWT), magnetostrictive sensors (MGSS), electromechanical impedance (EMI), electronic nose (e-nose), laser shock adhesion test (LASAT), Fourier transform infrared spectroscopy (FTIR), and nonlinear ultrasound (NLUS)

\begin{tabular}{|c|c|c|c|c|}
\hline User case & Process steps & Operations & Partner in charge & Used procedure \\
\hline \multirow[t]{13}{*}{ Production } & $\begin{array}{l}\text { Supplying } \\
\text { material }\end{array}$ & $\begin{array}{l}\text { Providing skin, } \\
\text { stringers, and } \\
\text { bonding }\end{array}$ & Aernnova, Airbus & \\
\hline & \multirow[t]{2}{*}{$\begin{array}{l}\text { Performing } \\
\text { contamination }\end{array}$} & $\begin{array}{l}\text { Contamination } \\
\text { applying RA }\end{array}$ & IFAM & Sweeping RA \\
\hline & & $\begin{array}{l}\text { Contamination } \\
\text { applying FP }\end{array}$ & & Salt-based solution \\
\hline & \multirow[t]{5}{*}{$\begin{array}{l}\text { Surface } \\
\text { assessment }\end{array}$} & $\begin{array}{l}\text { Testing on separate } \\
\text { stringers }\end{array}$ & IFAM & LIBS \\
\hline & & Testing on stringers & Automation & AWT \\
\hline & & Testing on skin & AGI & FTIR \\
\hline & & Testing on skin & AGI & e-nose \\
\hline & & Testing on skin & ENEA & e-nose \\
\hline & $\begin{array}{l}\text { Bonding of } \\
\text { stringers }\end{array}$ & Curing in autoclave & IFAM & Curing cycle \\
\hline & \multirow{4}{*}{$\begin{array}{l}\text { Bonding } \\
\text { assessment }\end{array}$} & Testing on skin & IMP-PAN & EMI \\
\hline & & $\begin{array}{l}\text { Testing on skin }+ \\
\text { loading }\end{array}$ & GMI & MGSS \\
\hline & & $\begin{array}{l}\text { Testing on skin's } \\
\text { rear face }\end{array}$ & CEA & NLUS \\
\hline & & Testing on both sides & CNRS & LASAT \\
\hline \multirow[t]{10}{*}{ Repair } & $\begin{array}{l}\text { Supplying } \\
\text { material }\end{array}$ & $\begin{array}{l}\text { Providing real } \\
\text { fuselage panels and } \\
\text { repair material }\end{array}$ & Airbus & \\
\hline & Scarfing & Scarfing for repair & GMI & Stepping \\
\hline & \multirow[t]{2}{*}{$\begin{array}{l}\text { Performing } \\
\text { contamination }\end{array}$} & $\begin{array}{l}\text { Contamination } \\
\text { applying DI }\end{array}$ & IFAM & Sweeping DI \\
\hline & & $\begin{array}{l}\text { Contamination } \\
\text { applying FP }\end{array}$ & & Skydrol oil \\
\hline & \multirow{6}{*}{$\begin{array}{l}\text { Surface } \\
\text { assessment }\end{array}$} & Testing & Automation $\mathrm{W}+\mathrm{R}$ & AWT \\
\hline & & Testing & AGI & FTIR \\
\hline & & Testing & AGI & e-nose \\
\hline & & Testing & ENEA & e-nose \\
\hline & & $\begin{array}{l}\text { Testing on a separate } \\
\text { panel }\end{array}$ & IMP-PAN & LIF \\
\hline & & $\begin{array}{l}\text { Testing on a separate } \\
\text { panel }\end{array}$ & IMP-PAN & Vibrometry \\
\hline
\end{tabular}


Table 5.2 (continued)

\begin{tabular}{l|l|l|l|l}
\hline User case & Process steps & Operations & Partner in charge & Used procedure \\
\hline \multirow{2}{*}{} & Bonded repair & $\begin{array}{l}\text { Preparing the } \\
\text { patches }\end{array}$ & GMI & Anita setup \\
\cline { 3 - 5 } & Preparing the curing & & \\
\cline { 3 - 5 } & $\begin{array}{l}\text { Curing outside the } \\
\text { autoclave }\end{array}$ & & \\
\cline { 2 - 5 } & \multirow{2}{*}{$\begin{array}{l}\text { Bonding } \\
\text { assessment }\end{array}$} & Testing on panel 1 & IMP-PAN & EMI \\
\cline { 3 - 5 } & Testing on panel 1 & GMI & MGSS \\
\cline { 3 - 5 } & Testing on panel 2 & CEA & NLUS \\
\cline { 3 - 5 } & Testing on panel 2 & CNRS & LASAT \\
\hline
\end{tabular}

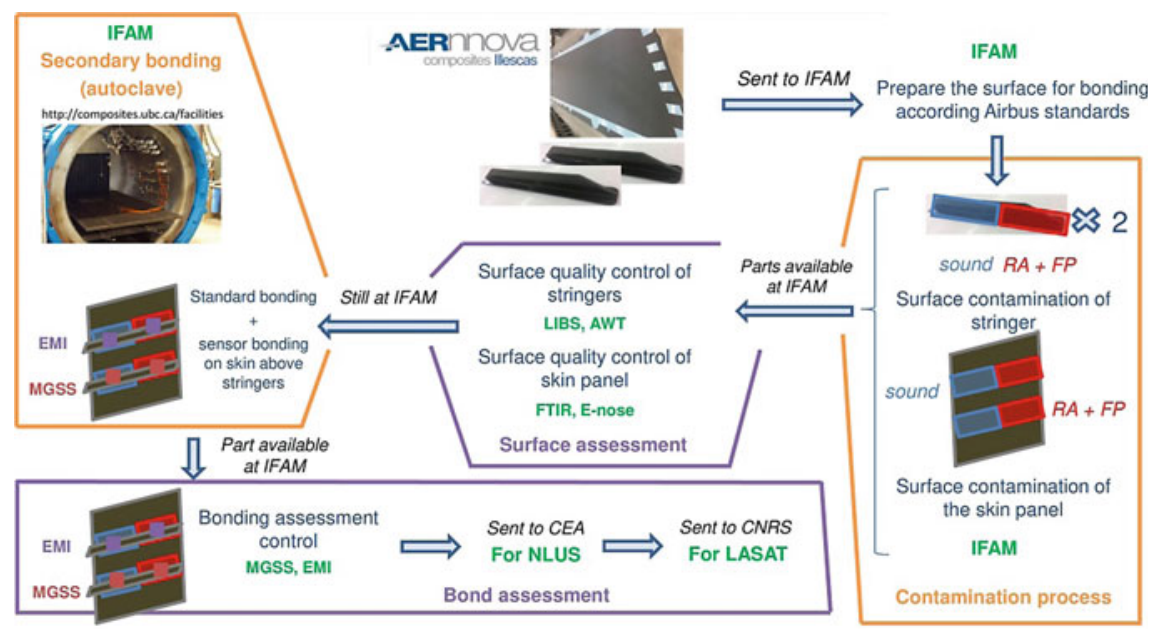

Fig. 5.6 The full-scale demonstration workflow for the panel used in the production user case

surface quality assessment. Considering that the e-nose techniques can potentially remove some contaminants during the measurements, separate areas were assigned for each e-nose technique. The stringer surfaces were also tested using AWT and (on a separate set of stringers) the LIBS technique. Indeed, as explored in the previous chapters, performing LIBS investigations can slightly modify the composite surface locally by vaporizing some of the epoxy-based matrix material. Therefore, any findings obtained with surface assessment techniques following LIBS testing are potentially influenced. After surface quality control, the stringers were bonded to the skin following a secondary bonding process in an autoclave. Hereby, the contaminated regions were placed above each other. After bonding, the obtained part was rapidly checked visually and manually to ensure that the demonstration tests did not focus on regions that were evidently not expertly bonded as this would have been trivially identified by the ENDT techniques. Subsequently, the bonding assessment was 
conducted. The magnetostrictive sensors were first bonded to the skin using an outof-autoclave device (see the repair user case section) before being used for the test. Then, the EMI sensors were glued to the skin in different regions to be used for testing during the final day of the full-scale demonstration after hardening overnight. Since the NLUS and LASAT devices were not available at IFAM in Bremen, the panels were sent to CEA to be tested first by NLUS and then to CNRS to be tested by LASAT, since the latter test might be destructive for weak regions.

\subsubsection{Release Agent and Fingerprint Contamination}

In this section, we focus on the important step of applying an intentional combined contamination during the three-day full-scale demonstration event within the ComBoNDT project. The specific operation is illustrated in Fig. 5.7. First, the position of the stringers was marked on the skin, following the initial design of the stiffened panel, as illustrated in Fig. 5.7a. The decision was made to situate the stringer at a distance of one-third of the height away from each border. Then, the panel was partially contaminated with a solution containing RA; the respective positions are displayed in Fig. 5.7b. The RA contamination was deposited along two strips using a small sponge, whereby the width of each strip was about the same as the stringer foot, as shown in Fig. 5.7c. The process was somewhat different from that used for the flat coupon samples, but in this realistic user case, it was not possible to use deepcoating due to the size of the part. As a consequence, there may have been a lower homogeneity on the surface, but we expected that the amount of Si species at the surface would turn out to be within the concentration range previously investigated

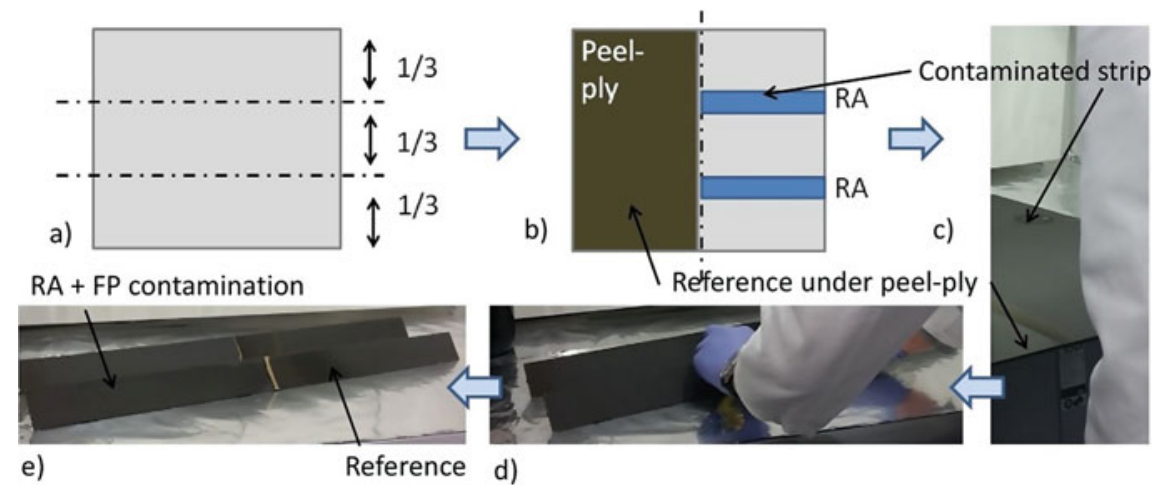

Fig. 5.7 The two-step contamination process "RA+FP" for the production user case; a position of the stringers, $\mathbf{b}$ regions to be contaminated with RA, $\mathbf{c}$ view of both areas contaminated with RA as well as the region where the FP contamination was randomly applied on the skin, $\mathbf{d}$ contamination of the stringer feet with RA, and $\mathbf{e}$ final contamination state of the stringer surfaces after the concluding deposition of salt-based FP 
using flat specimens. Following the RA contamination, the skin was placed in an oven for $1 \mathrm{~h}$ at $80{ }^{\circ} \mathrm{C}$. Subsequently, the FP contamination operation of the production user case was conducted in the same way as in the FP scenario for the coupon pilot specimens. We did not specify the distance between the applied prints in the contaminated region because we intended a random distribution, and their number should have been sufficient to be representative and unforeseeably positioned within the scanning areas of all the investigated techniques. It should be noted that during the whole contamination process, we retained the peel ply in the reference area to avoid any contamination of this region, as shown in Fig. 5.7. The stringers were then contaminated using the same process. RA was first spread on half of the finger foot surface using the same solution and a sponge (see Fig. 5.7d). They were then placed in the same oven, after which a worker marked the modified surface with FP deposits. The final state of the stringer surfaces was an "RA+FP" coated contamination area near a reference area below the peel ply, as presented in Fig. 5.7e.

After these contamination steps, the skin and stringer were ready to be tested using the various ENDT techniques. As explained, the stringers were dedicated to LIBS and AWT investigations while the skin was used for e-nose characterization. Clearly, from an ENDT point of view, this choice was arbitrary, however, it was made for operational reasons, mainly due to the geometry of the part. As we considered that the investigations with the e-nose technique might have removed some of the deposited substances from the stringer bonding surface, it was only tested by AWT (see Fig. 5.6). Thus, the stringer foot remained correctly contaminated after the first batch of ENDT and it was not necessary to repeat the contamination process.

\subsubsection{Bonding Operations}

After the testing of the different ENDT techniques with respect to their performance regarding surface quality assessment in a realistic production user case, the stringers were adhesively bonded to the skin.

The curing process of the stringers was quite conventional. Nevertheless, we would like to reiterate that we had decided on the techniques used in the bonding quality assessment under the consideration that one of the two adherend surfaces of each joint would be contaminated. Of course, in the curing oven, the contaminated region of each stringer was placed above the contaminated region of the skin to keep the reference bonding area away from the contaminants (as shown in Fig. 5.8a). We first applied the adhesive to the stringer foot and then positioned this onto the skin at the right place. We used adhesive tape strips to keep the partially contaminated stringer in place during loading and curing. The curing cycle in the autoclave was the same as that used for the coupon specimens.

After curing, the panel recovered from the autoclave contained both a reference area and a contaminated area, as illustrated in Fig. 5.8b. Thus, it was ready for use in the testing of the targeted set of ENDT bonding assessment techniques. In addition, to conventionally address non-expertly bonded regions of manufactured 

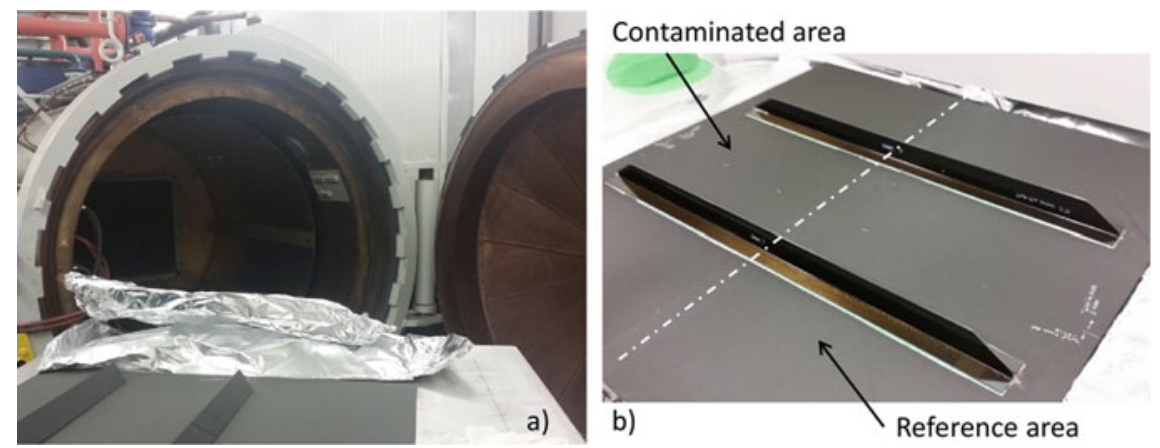

Fig. 5.8 Aspects of the bonding operation performed during the full-scale demonstration event in the ComBoNDT project: a panel and stringers in front of the autoclave before bonding and $\mathbf{b}$ fully cured stiffened panel

joints, we performed an ultrasonic inspection to check for unintentional defects in the bond line. This inspection revealed the presence of a region characterized by a low ultrasound amplitude, which most likely indicated some porosity. Such trivially defective regions were marked on the panel to be excluded from any subsequent in-depth ENDT investigation.

\subsection{Repair User Case}

\subsubsection{Workflow Overview}

This section describes the workflow for the repair application case in the ComBoNDT project, with the process illustrated in detail in Fig. 5.9. For several reasons, the decision was made to prepare three panels. First, the area to be tested on the repair specimens was smaller than in the case of the panel manufactured for the production user case, whereby we focused on conical repair regions with a diameter of about $20 \mathrm{~cm}$. This was a good compromise because such a region was large enough to represent a real application case yet also small enough to facilitate an easy and fast repair within the three-day full-scale demonstration event of the ComBoNDT project. For example, we had decided not to involve or touch the stringers in this particular user case. Second, the magnetostrictive sensors were positioned so as to capture a significant portion of the bond line. To independently test further bonding assessment techniques, we allotted additional space because we anticipated that this more complex bond geometry, compared to the specimen in the production user case, might require different settings for each step in the ENDT testing sequence. Thus, we dedicated one repaired panel to testing the intrusive bonding assessment techniques (MGSS, EMI) and another panel to the scanning techniques. In addition to these two 


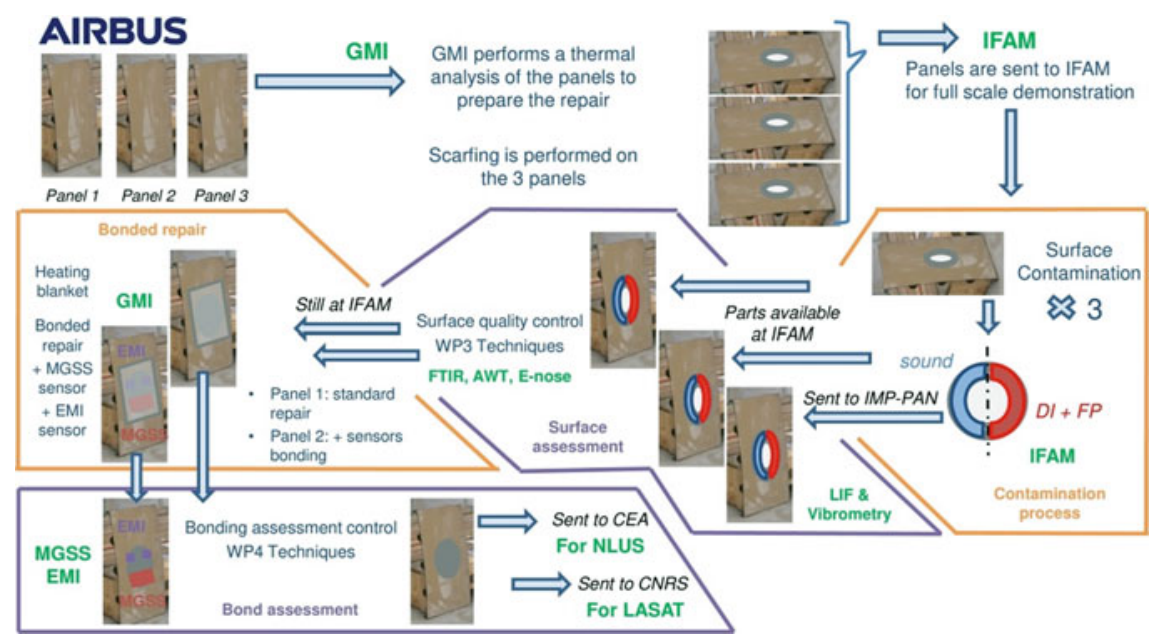

Fig. 5.9 Workflow description for the full-scale demonstration of the bonded repair user case

specimens, we agreed to provide a scarfed, but not repaired, panel for characterization using the LIF and laser vibrometry techniques, which were conducted at the IMPPAN laboratory in Gdansk.

The three panels were first sent to GMI AERO prior to the demonstration event. There, a thermal analysis of the panels was performed in order to correctly set up the bonded repair procedures to be conducted. Then, the panels were scarfed according to the standards (as detailed in the next section) and sent to IFAM in Bremen for the full-scale demonstration.

At IFAM, the first task was cleaning the scarfed area using an Airbus cleaning procedure designed for such a geometry. Since the used product was based on isopropanol, some time was scheduled to allow the area to dry prior to the next step, i.e., the application of the contamination. The three panels were contaminated according to the same process. One panel was saved for further surface investigation using LIF and vibrometry, while the other two parts underwent the regular ENDTassisted repair process. The surface of one contaminated adherend was tested using portable ENDT tools for surface quality control, namely AWT, FTIR, and e-nose, in that order. As a desorber device was used, some of the contaminants might have been removed during the e-nose investigation and thus the contamination process was repeated after the first of the two different e-nose techniques had been tested (by AGI-G or ENEA), as well as once more before performing the bonded repair. The panels were then successively repaired (as further detailed in the section on bonded repair). Magnetostrictive sensors were bonded onto one panel using an additional autoclave curing process. The EMI-based sensor bonding and respective investigations were performed before this final curing cycle and the corresponding MGSS testing. Finally, the last repaired panel was sent to CEA for NLUS measurement and finally to CNRS for LASAT. 
The bonded repair of an aircraft CFRP panel after a more or less complex application history is a sophisticated process. Furthermore, we decided to apply a technologically relevant procedure. The subsequent sections detail the operations that were successively performed within the scope of the project.

\subsubsection{Description of the Scarfing Operation}

We first describe the repair of the coated panel. Before any adhesive bonding for repair was performed, the paint system was completely removed and the area around the repair region was prepared. Generally, the borders of the repair hole were rounded off and the hole itself was tapered to provide the best load transfer once the repair patch was bonded in. The images taken during the repair process are shown in Fig. 5.10. Scarfing, or taper sanding, is usually achieved using a high-speed grinder powered by compressed air, while stepping is an alternative method for removing material in preparation for the application of a repair patch. In stepping, the overall angle of the cone wall is achieved by removing a precise area of material per ply of the composite laminate. This is a gentle process that prepares the damaged area for the application of a repair patch. It is imperative to follow all the repair manual guidelines, and significant skill and practice on the part of the repair technician are mandatory.

We performed the stepping process using the innovative tools designed by GMI AERO. We also benefitted from a Leslie machining kit, which is a dedicated piece
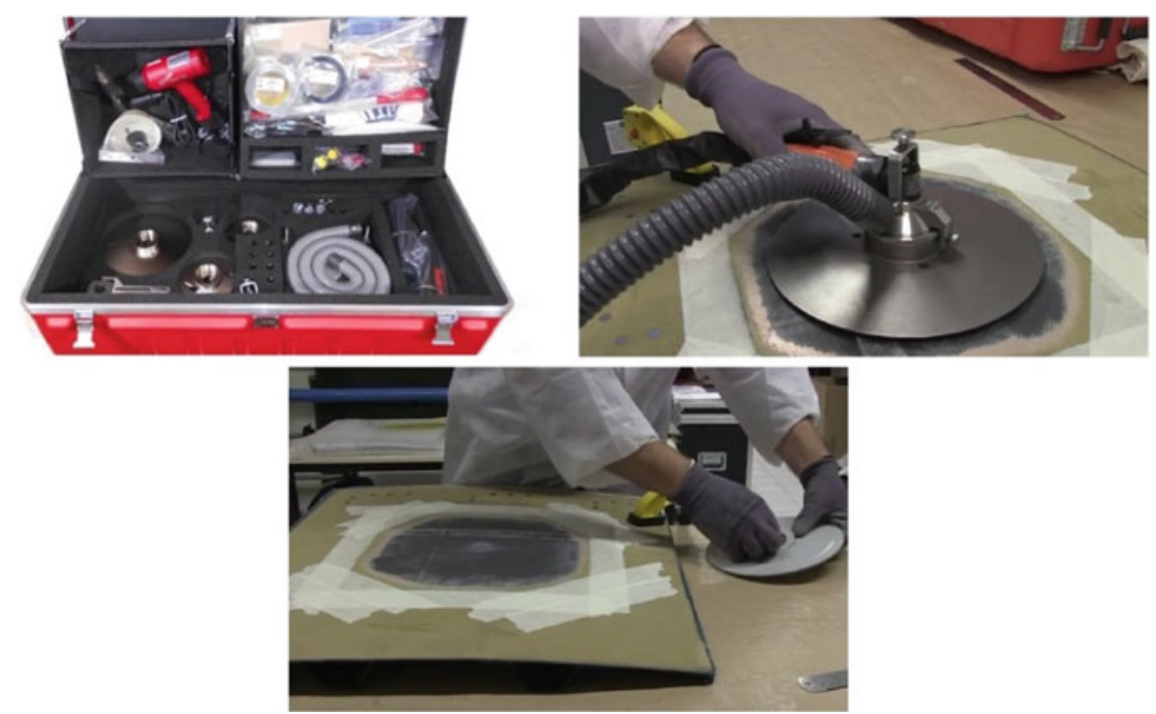

Fig. 5.10 Illustration of the stepping process prior to the application of a repair patch in the bonded repair user case 
of equipment used to prepare composite panels prior to the installation of repair patches. The following are the stepping characteristics chosen for this application:

- A circular repair geometry was used with a maximum diameter of approximately $200 \mathrm{~mm}$

- The average thickness of one ply was $0.15 \mathrm{~mm}$

- The overlap ratio was 1:60, i.e., a step width of approximately $10 \mathrm{~mm}$

- Seven steps were performed to achieve a depth of $1 \mathrm{~mm}$

All partners involved in the full-scale demonstration were informed of the thickness of the plies and their orientations. This was especially helpful whenever different settings had to be used per stepping step, e.g., in the case of LASAT, for which a setting per step was necessary.

\subsubsection{Application of the De-icer and Fingerprint Solutions}

This section provides more details about the contamination process. The images taken during the operations are presented in Fig. 5.11. Similar to the production user case, the repair user case comprised the intentional application of a combined contamination, although the respectively deposited solutions were different. Following the contamination scenario for the repair user case, the solution containing de-icing (DI) fluid was first applied. Similar to the RA contamination, a small sponge was used to spread the DI solution onto the stepped surface. Aluminum foil was then placed on the panel in such a way that it would keep half of the repair surface clean, as shown in Fig. 5.11a. As this was a manual operation and in view of the shape of the scarfing, some liquid might have moved towards the reference area. Thus, the boundary region between the reference and contaminated areas might have been

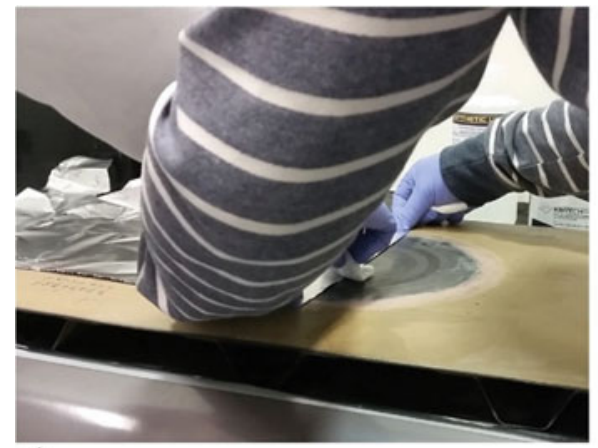

a)

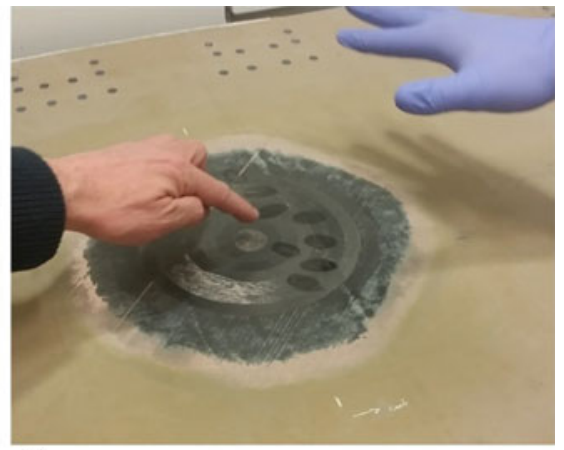

b)

Fig. 5.11 Contamination operation applied for the panels of the repair user case, with regionally applying contamination by a liquid de-icing solution while maintaining a contaminant-free reference area, and $\mathbf{b}$ a hydraulic oil solution deposited within the fingerprinted areas 
larger than was considered ideal, but this was deemed to be acceptable. After the contamination operation, the panel was placed in an oven for about 10 min. Fingerprints with Skydrol hydraulic oil were then manually placed on the contaminated surface, whereby a glove was used to achieve a better repeatability of the prints (see Fig. 5.11b).

As previously outlined, these operations were repeated twice during the fullscale demonstration event to restore the contamination deposit after each e-nose measurement. We took care to employ the same contaminants and worker from the first operation until the last to ensure a minimum deviation from one contamination operation to the next. After the last contamination step, the panels were ready for repair.

\subsubsection{Description of the Bonded Repair Operations}

The repair process involved the replacement of each carbon fiber layer that had been removed during the scarfing operation by a new fiber layer exhibiting an orientation that matched that of the original layer. Additionally, a film adhesive was placed between the structure and the carbon fiber layers, as shown in Fig. 5.12a.

After each prepreg layer was stacked, the curing facilities were installed on the panel, whereby a heating blanket was placed on top and capped by a vacuum bag, as depicted in Fig. 5.12b and c, respectively. The thermal curing was then performed using the appropriate temperature. For this application, we chose the adhesive film FM300-2M (supplied by Cytec) and the carbon prepreg material M20 (supplied by Hexcel). In the ComBoNDT project, we employed ANITA EZ0901 equipment (see Fig. 5.13) to bond the repair patch. The ANITA setup provides all the necessary functions to control the heating process under vacuum for bonded repair on composite

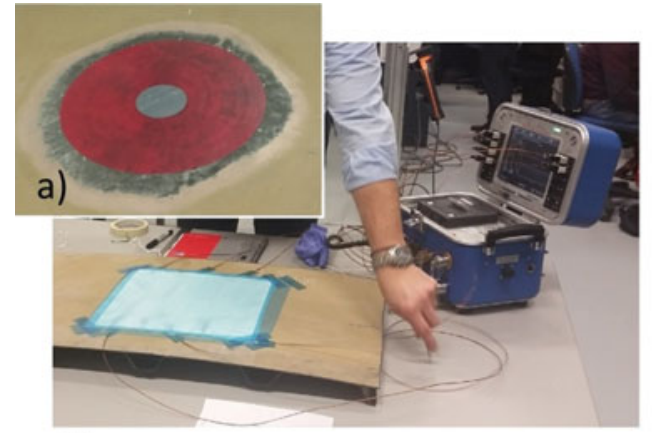

b)

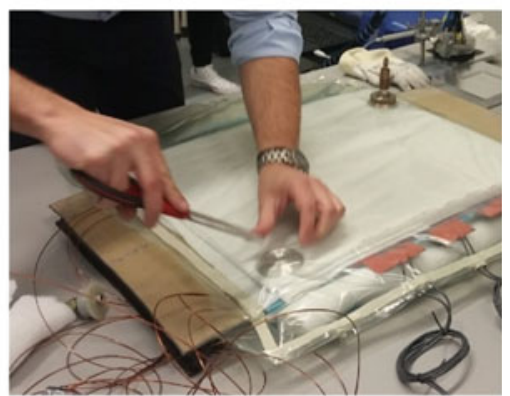

c)

Fig. 5.12 The distinct operations conducted during the bonded repair of CFRP panels showing a the raw F300-2 M bonding material, $\mathbf{b}$ the placement of the heating device, and $\mathbf{c}$ the preparation of the capping vacuum bag 


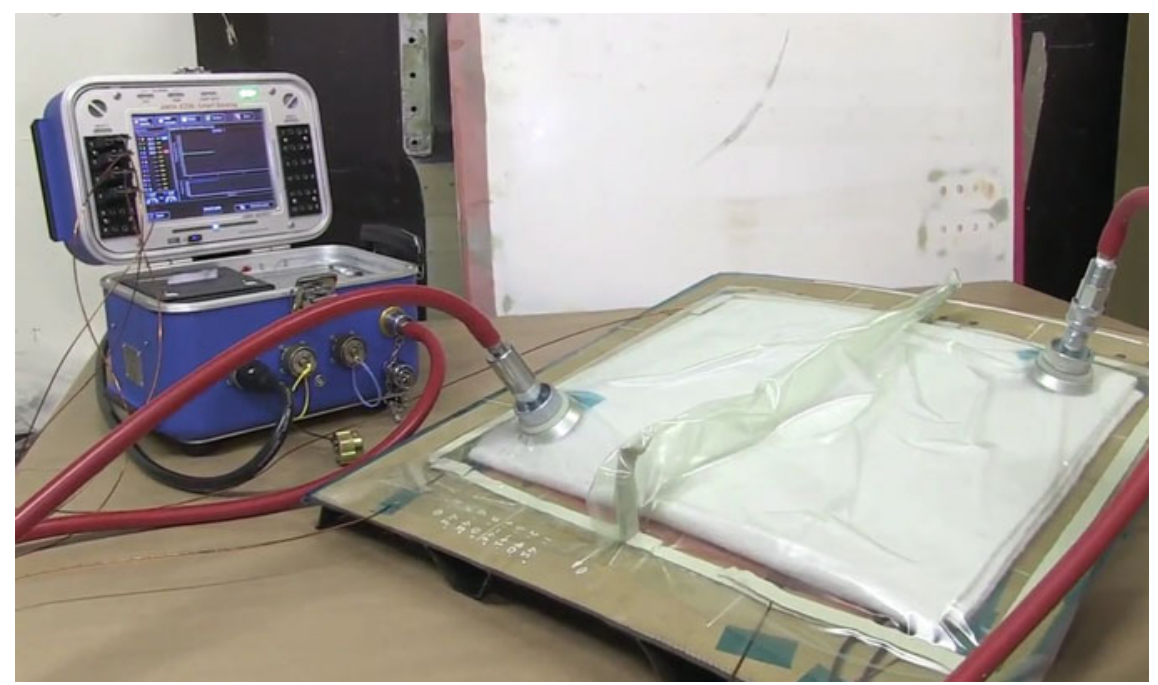

Fig. 5.13 Image taken during the full-scale demonstration of the repair process using the ANITA EZ0901 hot bonder in the ComBoNDT project

structures. The utilized device was specially conceived to achieve a maximum performance largely independent of the surface area to be repaired or the thickness of the assessed structures, and it is also suitable for structures with differentiated thermal reactions.

Appropriate curing of both the patch resin and the adhesive layer is essential to secure the integrity and sound mechanical performance of a bonded composite repair. A peculiarity of this user case, which focuses on Airbus A350 fuselage panels, results from the fact that these are carbon laminate structures reinforced with omegashaped carbon stringers. It should be noted that, when heating a patch for repair, this complex structure makes it very difficult to achieve an even temperature distribution. This is a well-known issue and in the past has been addressed through a multi-zone heating approach. However, as this process adds complexity and is time-consuming, a compromise was identified that took advantage of the structural periodicity in the case of the A350. To this end, the AdaptHEAT adaptive heating blanket was designed, tailored to A350 structural components, which could be applied using available twozone ANITA bonding consoles, thereby substantially improving the homogeneity of the temperature distribution. The AdaptHEAT concept adopts a multi-zone approach for the heating of a repair patch, following the heating requirements of the structure. In previous heating tests, we had identified that the part has two areas yielding different heating requirements, namely:

1. The area under which the stringers are positioned

2. The plain skin area, including the stringer foot. 


\subsection{Results of the Full-Scale Demonstration: The Representative Production User Case of a CFRP Stringer}

In this section, we present the findings obtained for the production user case during the three-day full-scale demonstration event within the ComBoNDT joint research project. First, we report on the surface quality assessment of the adherends in manufacturing a CFRP stringer, then we detail the outcomes of the bonding quality assessment after completing the joining process.

\subsubsection{Surface Quality Assessment}

In the following, we present the findings based on the LIBS, AWT, and e-nose inspections used for the surface quality assessment.

\subsubsection{Results for LIBS}

\section{Measuring setup}

The complex geometry of the stringer, i.e., a realistic part, as compared to the flat coupon samples required a substantial modification of the setup and an adaptation of the measuring process to facilitate the surface monitoring based on LIBS investigations. We achieved a significant increase in the TRL by developing a robot-aided quality assessment procedure that allowed both the sensitivity and the robustness of the standard laboratory-based approach to be maintained. Essentially, we matched the control of the LIBS sensor to that of a standard industrial robot that was set to handle and position the part to be analyzed, as shown in Fig. 5.14. This arrangement offers some advantages for the LIBS measurement process since the focal point of the LIBS laser can be fixed. This fixed focal point (as shown in Fig. 5.15) was used as the origin of the coordinate system with which the robot handled the parts. Focusing

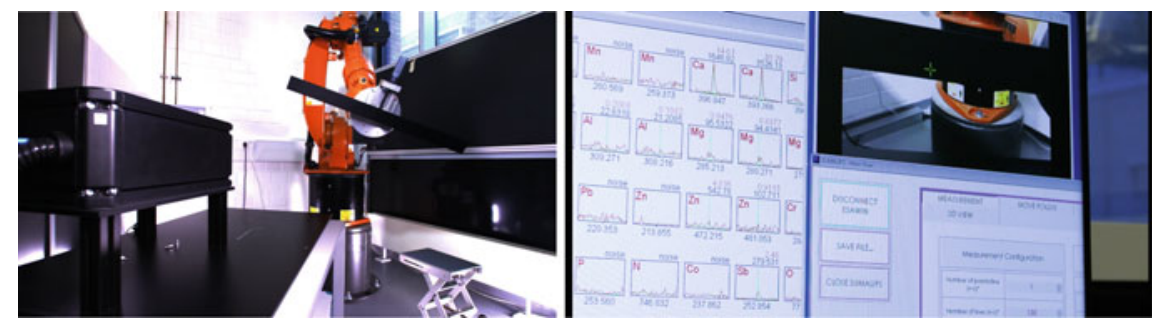

Fig. 5.14 The handling of a stringer by a fine-tuned industrial robot (left) and the well-arranged control unit for the operator (right) 


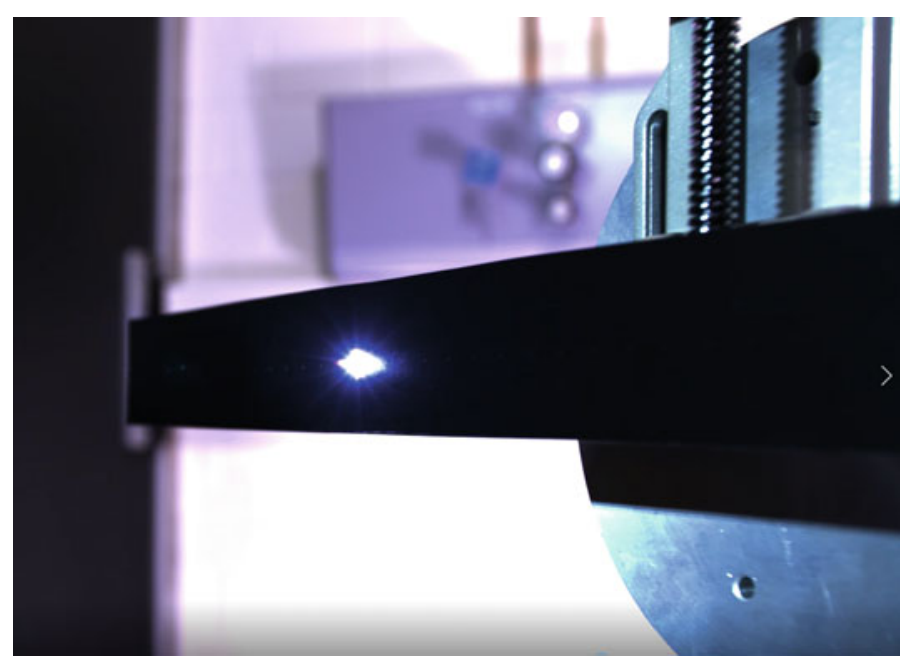

Fig. 5.15 The plasma generation during the LIBS measurement on the realistic stringer specimen

on the analytical process, the LIBS measurements were performed on the CFRP surfaces after the peel ply was removed and the defined combined contamination process was performed. A laser wavelength of $1064 \mathrm{~nm}$ was used with a $180 \mathrm{~mJ}$ laser pulse energy. In order to determine the surface composition of the stringer, surface areas along three lines-each comprising 130 single measurement spotswere analyzed. An image of such a stringer is depicted in Fig. 5.16, while selected LIBS signals obtained during the investigation are presented and discussed based in Fig. 5.17.

\section{Summary of the results}

In Fig. 5.17, we present the results achieved when aiming for the detection of a contamination that arose from depositing relevant amounts of a Si-containing RA on the C-rich stringer surface. Essentially, the first 65 measurement points obtained within the intentionally contaminated half of the stringer surface indicated a clearly increased $[\mathrm{Si}] /[\mathrm{C}]$ concentration ratio. This significant finding is indicative of the presence of RA contamination and is, as anticipated, in contrast to the LIBS results from the non-contaminated right half of the stringer surface. Thus, using LIBS

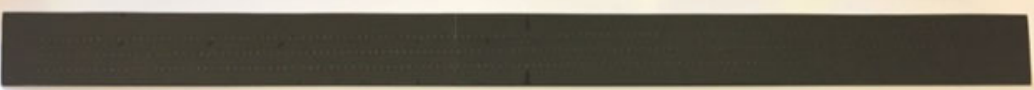

Fig. 5.16 An overview of the stringer surface after performing spot-by-spot LIBS measurements along three parallel lines, with the top, middle, and bottom lines composed of 130 analysis spots each; the left part of the specimen was contaminated, while the right part provided a clean reference surface 


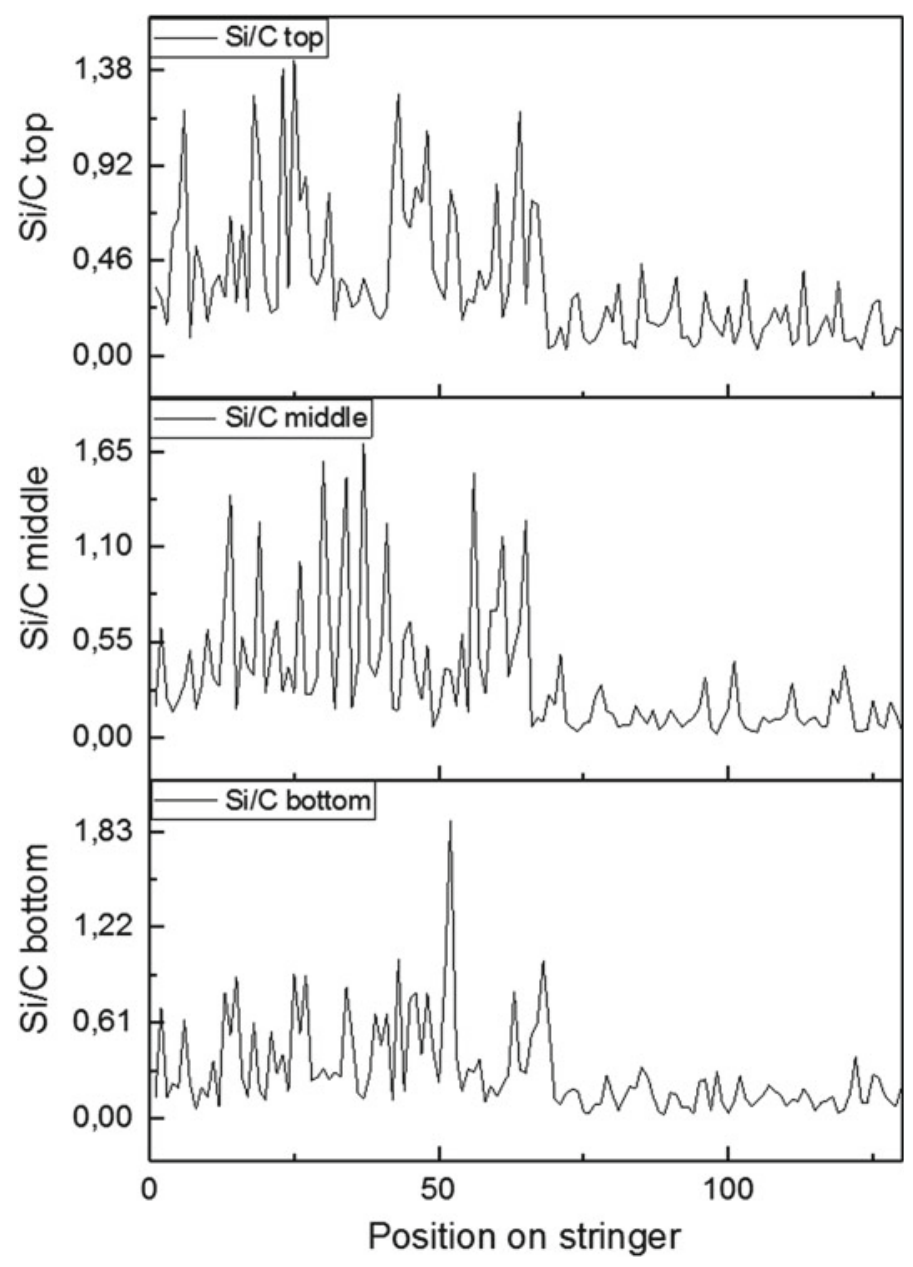

Fig. 5.17 LIBS findings from three horizontal lines composed of 130 positions on a partially contaminated CFRP, highlighting Si/C signal ratios measured on the composite surface in the contaminated area (left) and the clean reference area (right)

measurements it is clearly possible to detect and allocate the areas corresponding to either the contaminated or clean CFRP surface state. Additionally, evaluating further signals enables the type of contamination to be identified. Figure 5.17 presents the characteristics of the Na signal along with one of the lines composed of 130 measurement spots separated by $5 \pm 1 \mathrm{~mm}$, whereby it is possible to distinguish a locally increased intensity within the first 65 measuring points. Single peaks demonstrating an increased Na concentration indicate the presence of a Na-containing FP deposit in the contaminated area. It is more challenging to detect and locate the FP contaminations as the contaminated area was relatively limited compared to the region covered 
by the broader RA deposits and also because only up to three single LIBS shots were located in each FP contaminated area (Fig. 5.18).

In summary, when using the advanced robot-aided LIBS characterization process and evaluating both the $[\mathrm{Si}] /[\mathrm{C}]$ signal ratio and the $\mathrm{Na}$ signal intensity along three parallel lines composed of measurement spots separated by $5 \pm 1 \mathrm{~mm}$, we achieved a clear and significant differentiation between the clean surface area and the contaminated area of the stringer, the detection of the elemental constituents of the applied contaminations, and consequently the identification of both contaminants contributing to the combined deposit.

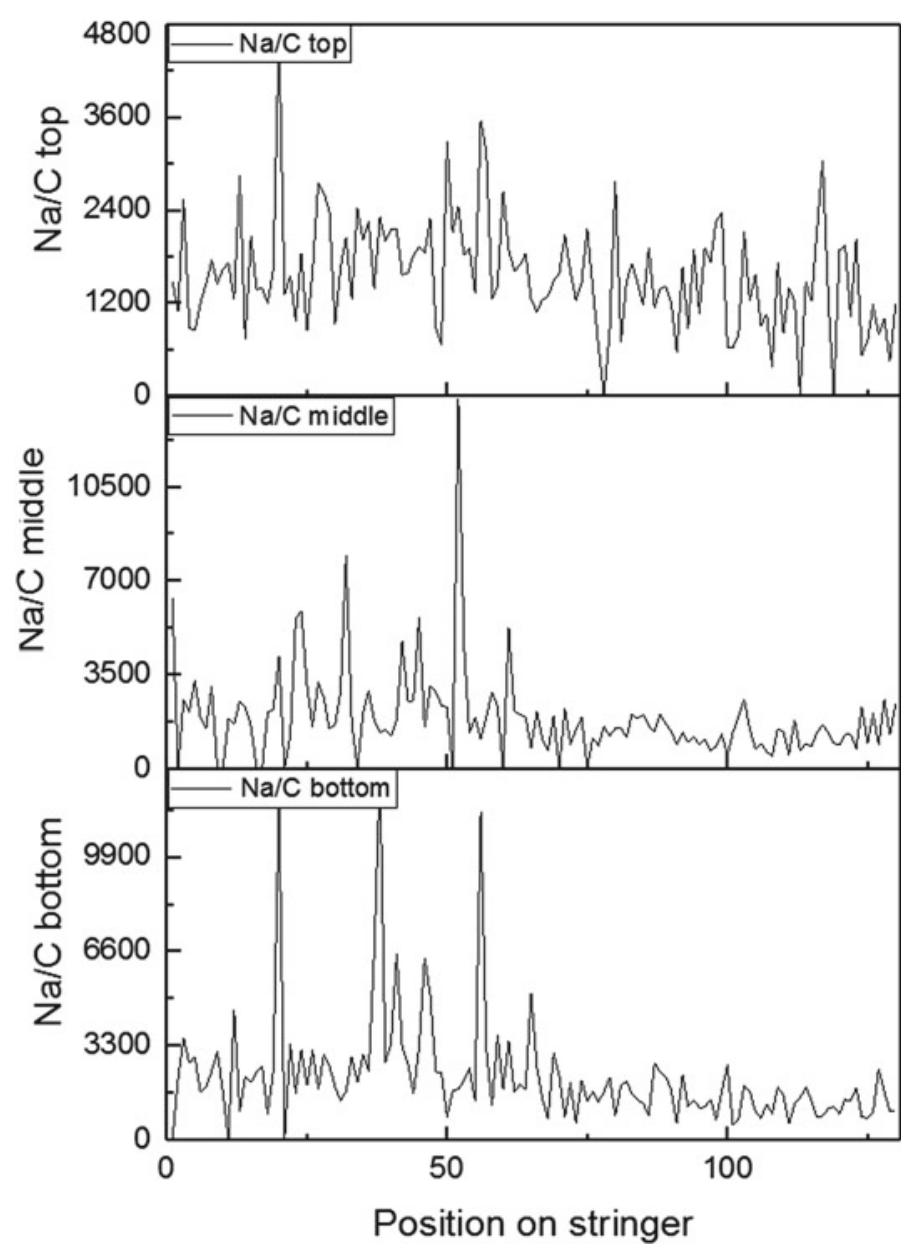

Fig. 5.18 Sodium signals for the three lines measured on the stringer surface give an idea about the contaminated areas (left) and the clean reference area (right) 

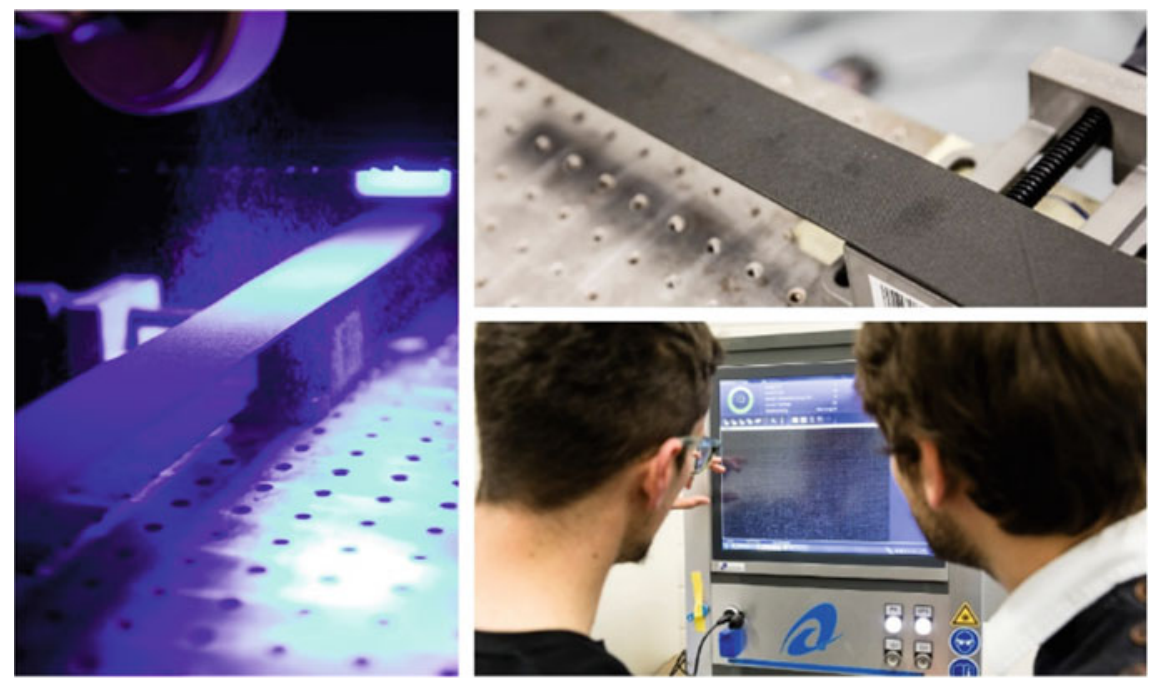

Fig. 5.19 An overview of the AWT-based investigations of a stringer surface during a full-scale demonstration

\subsubsection{Results for AWT}

The contamination on the production part was a single RA contamination on half of the part. This contaminated area was successfully detected and differentiated from the clean half. We were thus able to master the challenge of identifying and detecting laterally separated water droplets with the use of image processing based on a neural network. An overview of the measurements is given in Fig. 5.19.

\subsubsection{Results for E-nose}

Due to the high potential of e-nose techniques with respect to surface quality assessment, we tested the two distinct setups advanced in the ComBoNDT project during the three-day full-scale demonstration event.

\section{AIRBUS E-nose setup}

During the demonstration, the realistic and real parts of both main user cases were measured both with and without contamination. For the production user case, three different specimens were tested (a sample with the dimensions $20 \mathrm{~cm}$ by $20 \mathrm{~cm}$ and two production panels). Panel 1 provided a section with an intentionally contaminated area (Panel 1c) that had been prepared following the combined "RA-FP" scenario. All other samples were not contaminated. 
Figure 5.20 shows the desorber device, which was developed in the ComBoNDT project by the partners from Airbus, during its application in taking measurements on the realistic parts in the production user case.

Subsequently, we discuss the evaluation of the acquired e-nose data considering two different approaches to data treatment and representation, namely the partial least squares discriminant analysis (PLS-DA) model and the principal component analysis (PCA).

When we evaluated the measured data of the production panels using the PLS-DA that had been obtained from the pilot sample test, we achieved the differentiation and correct classification of all surface states in both the "clean" and "not clean" (i.e., contaminated) classes. In Fig. 5.21, the horizontal threshold line represents the boundary signal level that can be used to determine whether a data point is indicative of a panel surface state that is attributed to the "clean" class (i.e., ready for bonding) or the opposite class (class threshold).

By performing cross-validation with the obtained, the confusion matrix shown in Table 5.3 is obtained.

Concerning the data evaluation based on a single-class identification method, a different method of signal processing was tested to identify the true class with a clean surface state of a specimen panel. In detail, six samples from the sampling areas on the clean Panels 1 and 2 were taken to determine the region in the PCA that correlates to a clean sample surface. We used these samples to build the detection model. Using a confidence level of $95 \%$, we obtained the green domain shown in Fig. 5.22, which indicates the region in which an unknown sample would be classified as clean. Measurements that lie outside this domain would be classified as false, i.e., contaminated or not clean.

When we subsequently evaluated the remaining seven e-nose measurements of Panel 1 and Panel 2 with this built (single-class identification) PCA model, all measurements made on the uncontaminated areas of the panels were located inside the green domain. Meanwhile, all measurements made on the contaminated areas lay outside this domain, which represents clean surface areas, and were, therefore,

Fig. 5.20 The e-nose desorber device taking measurements on the CFRP Panel 1 within the production user case during the full-scale demonstration event

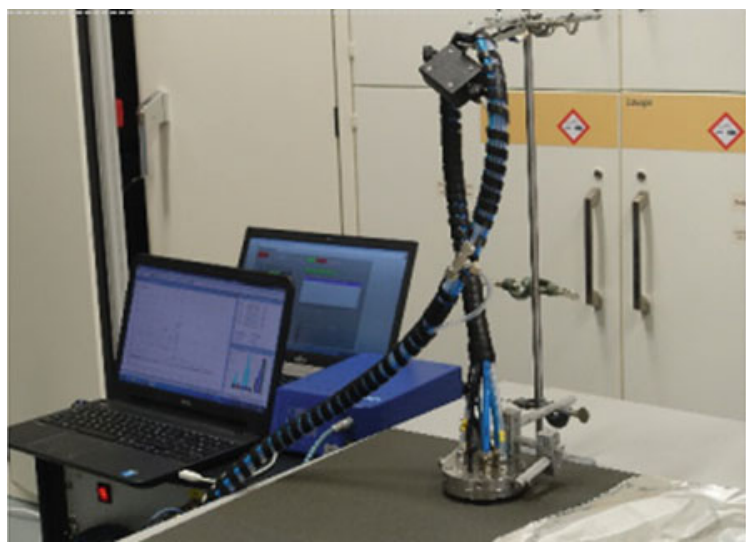


Fig. 5.21 Results of the class assignment of PLS-DA-treated e-nose data obtained on the technologically realistic parts of the production user case

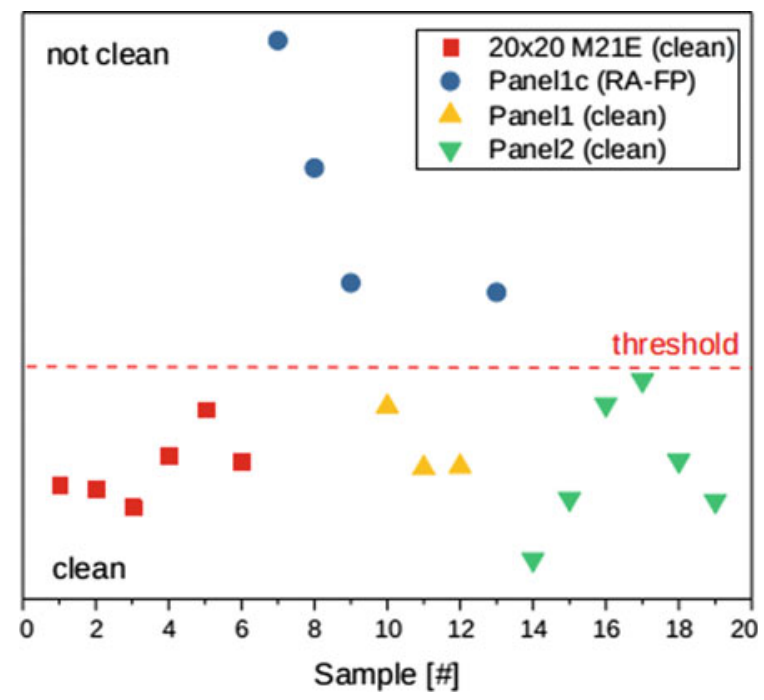

Table 5.3 Confusion matrix obtained when cross-validating the treated e-nose data shown in Fig. 5.21

\begin{tabular}{l|l|l}
\hline Actual class & REF & RA-FP \\
\hline Predicted as REF & 15 & 0 \\
\hline Predicted as RA-FP & 0 & 4 \\
\hline
\end{tabular}

correctly identified as being indicative of contaminated samples. Figure 5.22 displays all the results for the single-class identification evaluation. We would like to highlight that all "unknown" (i.e., prepared and analyzed on-site) surfaces of Panel 1 and Panel 2 were classified correctly based on the performed intentional contamination procedure and following the e-nose measurements.

\section{ENEA setup}

The realistic part production user case was also tackled using the latest version of the ENEA e-nose, which is depicted in Fig. 5.23.

Here, we briefly describe the developed data acquisition and evaluation process. Separate models for anomaly detection were produced in order to differentiate contaminated samples from the clean reference samples. Based on nine measurements taken from a (clean) curved realistic reference specimen, we obtained a low dimensional projection using the first three principal components in the PCA rotated sample space. Hypothesizing a multivariate Gaussian distribution of the measured data's projection on the low dimensional subspace, we used a two sigma coverage to set an ellipsoidal surface acting as a detection threshold for contaminated samples. Results from the measurements of clean surface regions were uploaded to the joint Graphical User Interface (GUI) software to compute the detector surface and prepare the GUI to accept the test measurements. Then, we used findings from nine sample regions to test the developed detector: three from clean areas of the tested part and six 


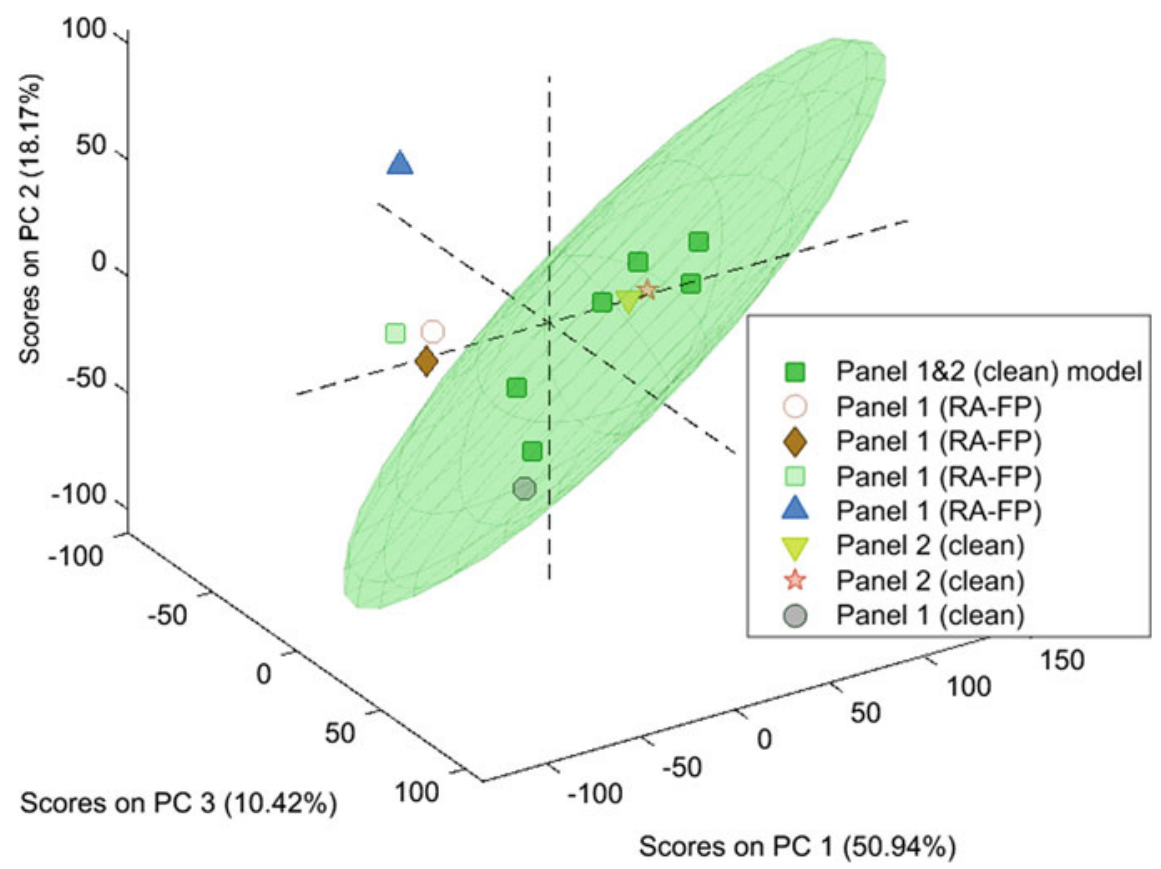

Fig. 5.22 Single-class identification of e-nose results following PCA-based data evaluation from measurements on technologically realistic parts. The green domain indicates the region of measurements on clean samples with a confidence level of $95 \%$

Fig. 5.23 An ENEA e-nose employed for inspection of a technologically relevant realistic composite part during the full-scale demonstration event

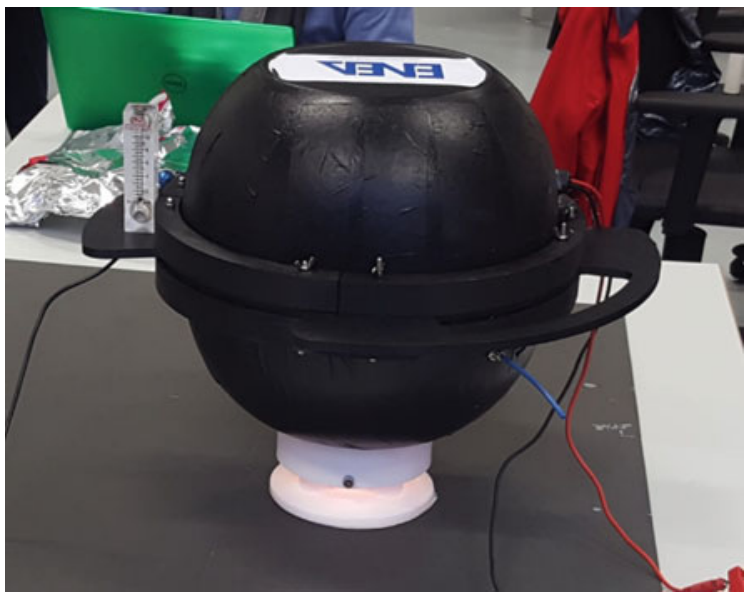


from areas that had been contaminated previously following the "RA-FP" scenario. In summary, we measured 18 samples, of which nine were set apart to build the training set of the PCA-based detector, while the other nine were used for testing purposes. The joint GUI was able to show the results in real-time immediately after the measurement was taken. A screenshot of the active GUI is displayed in Fig. 5.24.

Table 5.4 presents the confusion matrix summarizing the results obtained for the production sample scenario.

As can be seen in Table 5.4, based on the advanced process, we achieved a perfect recognition score. Furthermore, the detection threshold was overcome along a similar direction in the same sub-plane for all except one of the contaminated samples, which adds robustness and significance to the results.

\section{Synopsis: The findings for surface quality assessment within the production user case}

The LIBS measurement technique has successfully shown its potential in monitoring composite adherend surfaces, even in the case of complex shapes. During the threeday full-scale demonstration event in the ComBoNDT project, we used the technique for the automated measuring and detection of multiple contaminations with RA

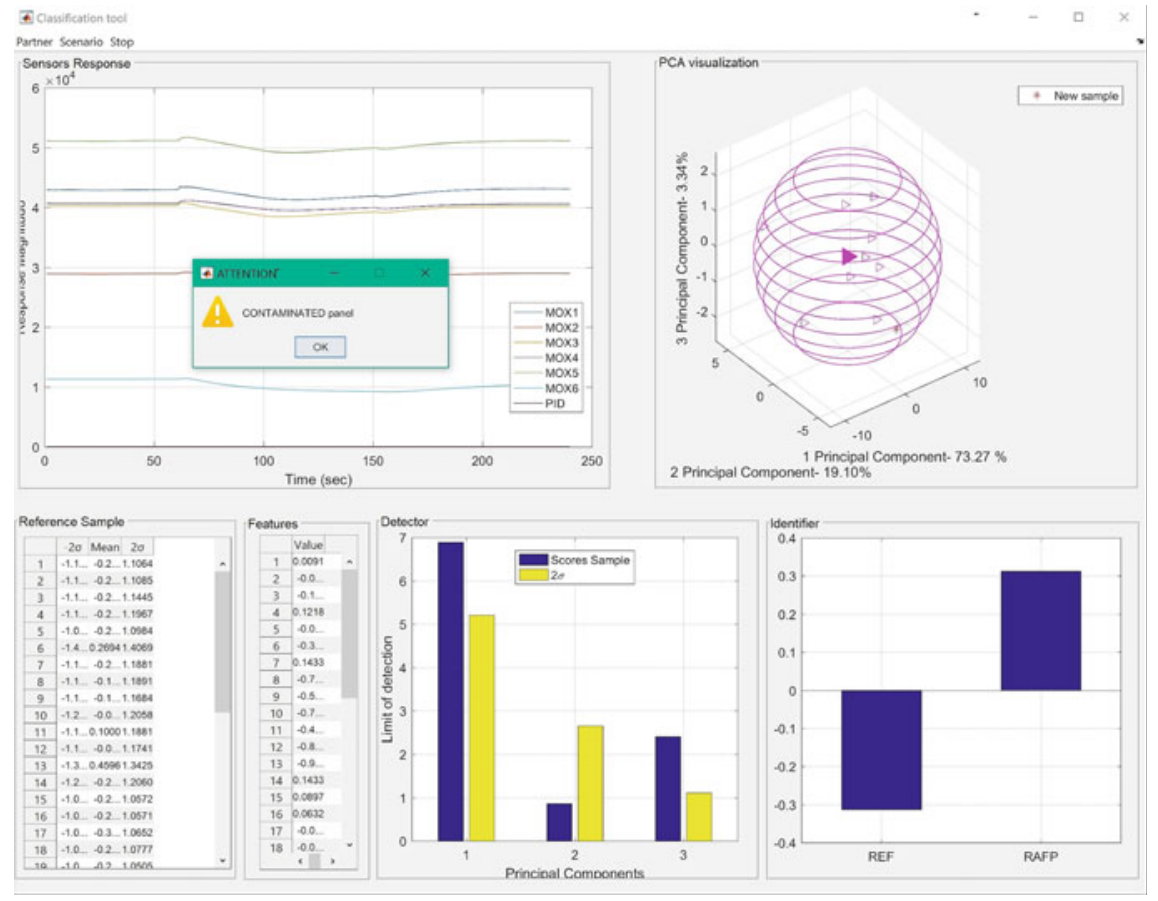

Fig. 5.24 Screenshot of the ENEA GUI employed for the detection and identification tasks based on the e-nose investigations of contaminated realistic CFRP parts within the production user scenario during the full-scale demonstration event 
Table 5.4 Confusion matrix obtained when employing the ENEA e-nose for contamination detection on realistic composite parts from the production user case

\begin{tabular}{l|l|l}
\hline Actual class & REF & RA-FP \\
\hline Predicted as REF & 3 & 0 \\
\hline Predicted as contaminated & 0 & 6 \\
\hline
\end{tabular}

and FP, whereby both contaminants were detected and revealed. If a more clearly localized detection of single contaminant-based FP were aspired in the future, then a higher statistical basis, i.e., more measuring points than the 390 measured here for each stringer, would be required.

Using AWT to inspect a realistic production CFRP part, a single RA contamination was detected by means of advanced data evaluation based on a neural network. Finally, a robust and significant detection of a complex combined contamination was achieved using both distinct enhanced e-nose techniques employed for surface quality assessment during the full-scale demonstration event, applying PCA for data evaluation and benefitting from a user-friendly GUI.

\subsubsection{Bonding Quality Assessment}

In the following, we present the findings based on the EMI, NLUS, and LASAT inspections used for bonding quality assessment in the production user case assessed during the three-day full-scale demonstration event within the ComBoNDT project.

\subsubsection{Results for EMI}

The realistic part prepared for the production user case was a CFRP panel with an adhesively bonded stringer, as depicted in Fig. 5.25. One half of the bonding area for the stiffener was intentionally contaminated before bonding. The rear side of this region is shown in the left part of Fig. 5.25a. The combined contamination scenario comprised the application of RA and FP. The second half of the stringer surface was maintained in a clean state and its rear side is shown in the right part of Fig. 5.25a. Technically, within the EMI-based inspection process, this area is used for reference purposes.

Specifically, we bonded two piezoelectric sensors with cyanoacrylate glue to the adherend surface of the CFRP panel (Fig. 5.25a) in order to assess the bonding quality of its joint with the stringer. We placed the sensors on the rear side of the panel in the area where the stringer was bonded. The DD1 sensor was placed on the area of the clean bond, while the DD2 sensor was bonded to the contaminated (following the "RA-FP" scenario) region. The electrical characteristics of both sensors were measured three times. First, the free sensors were measured for referential purposes. 


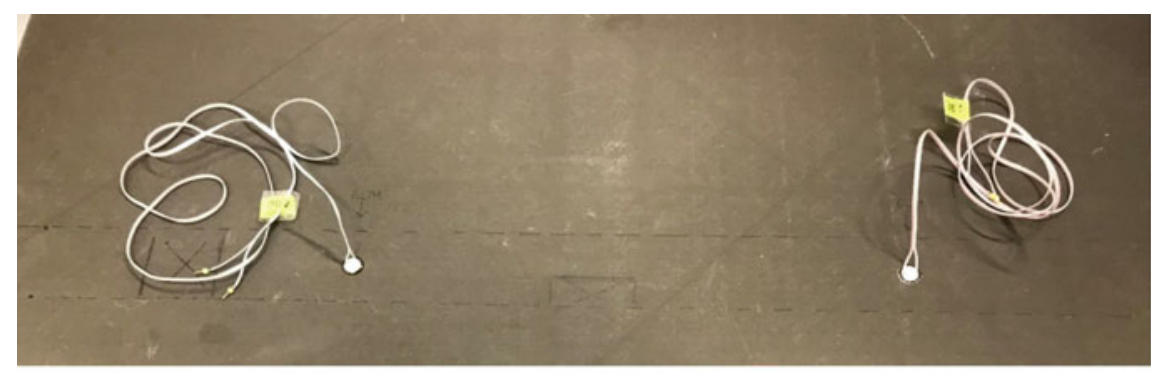

a)

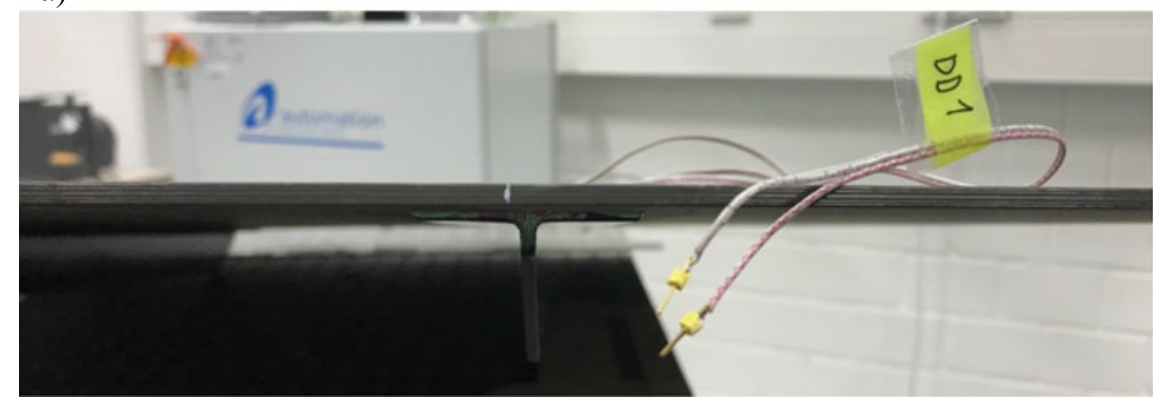

b)

Fig. 5.25 The realistic CFRP panel used as one partially contaminated adherend within the production user case, with a the piezoelectric sensors for EMI measurements and $\mathbf{b}$ the stiffener bonded to the front side. In (a), the sensor on the left-hand side (DD2) is bonded to a contaminated surface area and the sensor on the right-hand side (DD1) is bonded to a clean adherend area

Second, the bonded sensors were measured while the panel was lying on the laboratory table (as depicted in Fig. 5.25b). Third, a measurement was made while the panel was being subjected to the external loading situation used for strain measurements. The loading was applied by attaching the panel to the laboratory table with adhesive tape, as demonstrated in Fig. 5.26.

With respect to the sensor signal output and the subsequent data evaluation, Fig. 5.27a presents the obtained root mean square (RMS) values for the conductance obtained in the 3-5 MHz frequency range. After bonding the sensors, a drop in the RMS value was obtained as compared to the free sensors. We observed higher RMS values for the sensor bonded to the contaminated area, which may indicate a weaker adhesive bond. Additionally, when applying an additional load to the panel, the two sensors showed significantly different signal changes. We observed an RMS increase for the clean region, while a decrease was found for the contaminated bond region. As a second characteristic, the conductance maximum peak shift was also tracked, as displayed in Fig. 5.27b. Taking as a reference the frequency at the conductance maximum of the freely oscillating sensor to be bonded close to the bonding region with the clean adherend, the difference to the frequency at the conductance maximum of the second freely oscillating sensor was below $0.5 \%$. After bonding both sensors, a significant increase of this characteristic frequency was observed, 


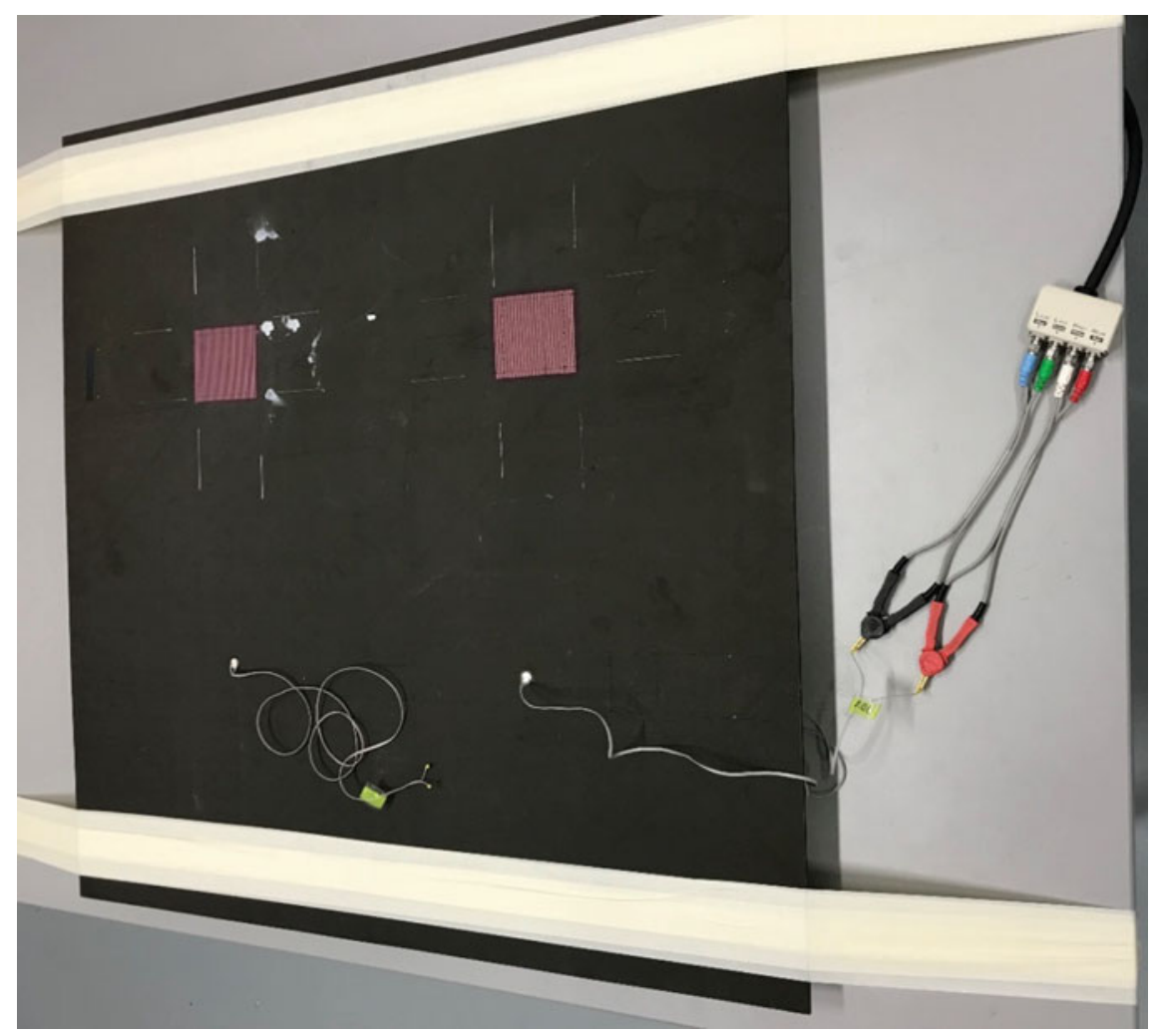

Fig. 5.26 The curved realistic part for the production user case with a loading situation introduced by fixing the CFRP panel in a flattened adjustment using adhesive tape. The square patches with pink color are strain sensors introduced by GMI AERO

which was more pronounced for the bond comprising the contaminated adherend. After mechanically loading the panel, the peak for the clean bonding region shifted leftward towards a lower frequency, while the one for the contaminated region shifted rightward, reaching a shift of almost $2 \%$.

After inspecting the measured conductance curves in more detail, local resonances in the range of 3.75-3.90 MHz were observed. These affected the RMS values calculated for this frequency region as compared to the RMS values for the free sensors (see Fig. 5.28a). Similar behavior was observed for the resistance curves but in a slightly different frequency range, namely from 3.6 to $3.9 \mathrm{MHz}$, as shown in Fig. 5.28b. As compared to the regions close to the bonds with clean adherends, we observed lower RMS values around the contaminated area, which may indicate a weaker adhesive bond there. 


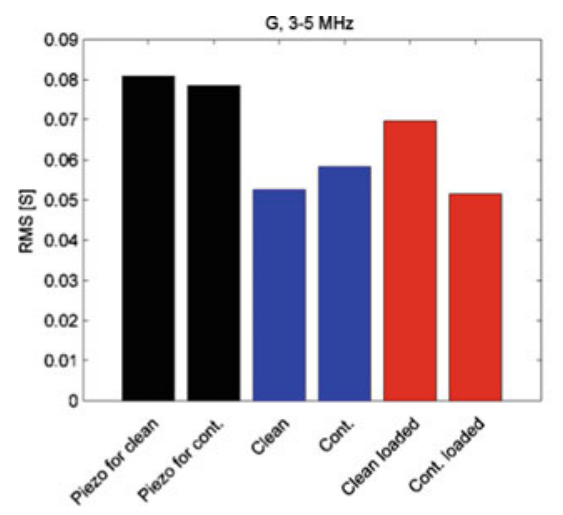

a)

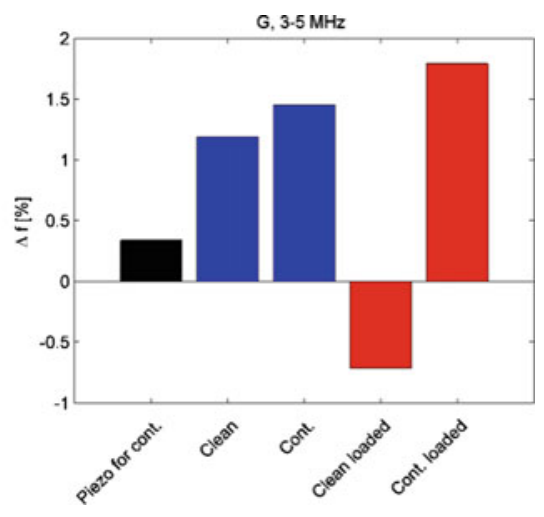

b)

Fig. 5.27 The EMI results for the six investigated bonding states for realistic parts from the production user case obtained in the 3-5 MHz frequency region; a RMS values of conductance and b frequency shift of the conductance peak

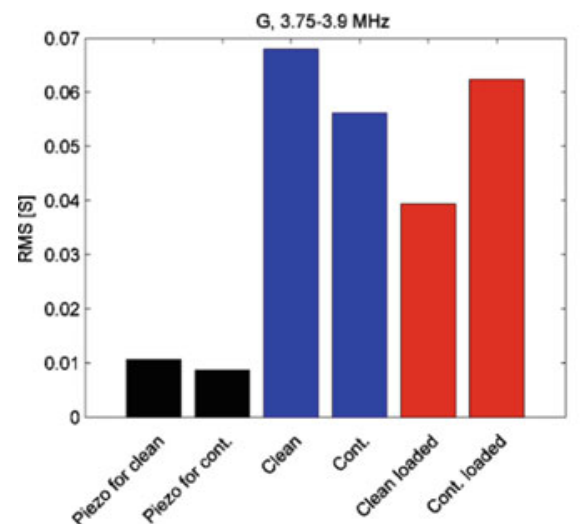

a)

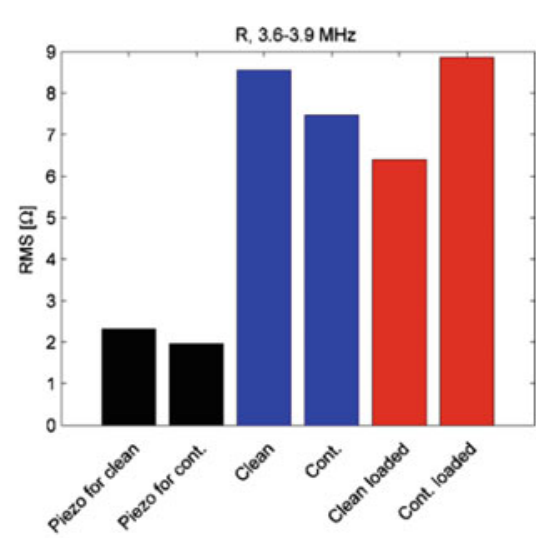

b)

Fig. 5.28 Further details of the EMI findings revealing local resonances in the RMS values of the a observed conductance signals and $\mathbf{b}$ resistance curves

\subsubsection{Results for NLUS}

For the investigation of the bonded CFRP specimen from the production user case, the ultrasound excitation transducer was positioned symmetrically in relation to the longitudinal extension of the stringer to be tested, as shown in Fig. 5.29. Such a position provided a symmetrical insonation field for this stringer, and the findings for the fundamental frequency $20 \mathrm{kHz}$ are displayed in Fig. 5.29. 


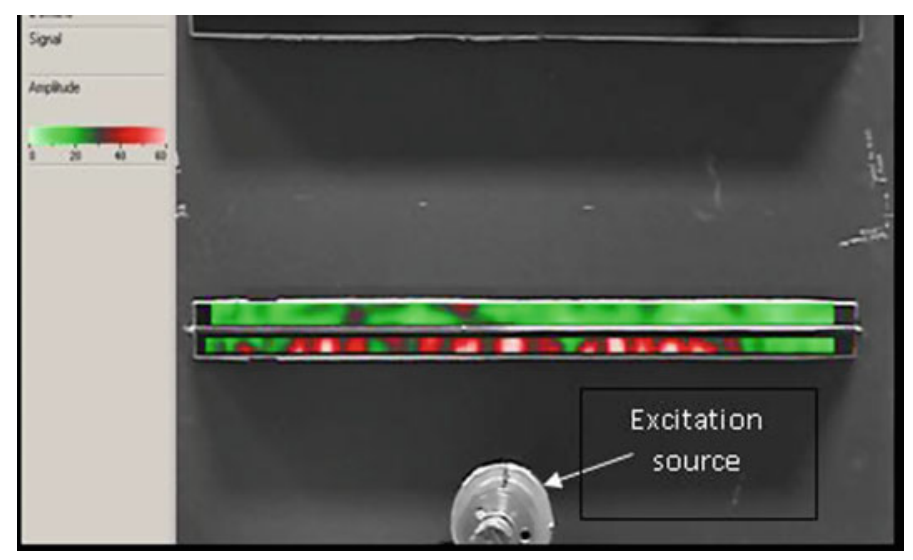

Fig. 5.29 Distribution of the fundamental ultrasonic frequency $(20 \mathrm{kHz})$ amplitude along the stringer in the realistic bonded CFRP specimen from the production user case, as observed during the NLUS inspection

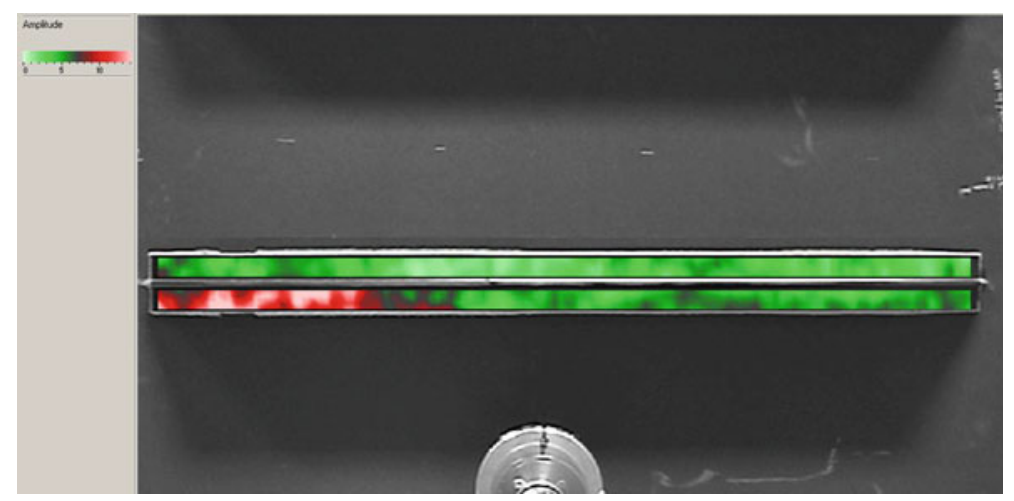

Fig. 5.30 Distribution of the second-highest harmonic $(40 \mathrm{kHz})$ along the stringer length in the bonded production CFRP component, as observed during the NLUS inspection

Additionally, the second-highest harmonics were measured in the production specimen. The findings shown in Fig. 5.30 demonstrate quite different signal distributions, whereby the higher efficiency of the local nonlinear generation in the lefthand-side of the stringer can be clearly perceived and identifies a bonding region based on a contaminated adherend surface area with a lower bonding quality.

\subsubsection{Results for LASAT}

Before detailing the results obtained when applying the laser shock adhesion test (LASAT) for investigating the realistic bonded CFRP specimen from the production 
user case, we provide a short and demonstrative introduction to the full-scale test performed during the demonstration event of the ComBoNDT project.

\section{Introduction to the full-scale test}

In order to facilitate the LASAT investigations on large-area realistic or real parts with complex, e.g., curved, shapes, we advanced the Hephaïstos setup by implementing profound modifications. With the aim of handling specimens of the required size, the optics had to be substantially rearranged and a robot had to be installed to manipulate the sample. The resulting setup is displayed in the on-site environment in Fig. 5.31.

The second challenge was the confinement. So far, the plasma generating the shock wave within the sample had been confined using a thin water layer. Even though this ENDT technique offers an easy setup on a smaller scale, the new upscaled configuration would have introduced substantial operating costs, e.g., the installation of special pipes to supply the investigated sample region with water as well as to ensure water drainage. To overcome these challenges, solid confinement was used. Numerous materials were tested, and for some of these, the results for the flux-dependent plasma pressure are displayed in Fig. 5.32. This graph describes the generated pressure as a function of the laser intensity for confinements based on three different materials. The LASAT operating regime is around $1-3 \mathrm{GW} / \mathrm{cm}^{2}$, and in this flux interval, water, epoxy, and a thick transparent PPE adhesive tape turned out to have the same confinement capability. With respect to the aspired application, the latter was eventually chosen for its ease of use, and the setup is shown in Fig. 5.33. The aluminum sacrificial layer was not modified.

Briefly, the workflow for testing was as follows. For each sample, an initial threshold identification was performed in different areas. On each calibration area, a series of laser shots were performed. If no damage was inflicted, the laser intensity over the area was increased. The opening energy of a sound part was defined as the "reference threshold". Once this threshold had been identified and confirmed for

Fig. 5.31 The advanced Hephaïstos symmetrical LASAT setup with a robot to manipulate the bonded CFRP specimen in the production user case

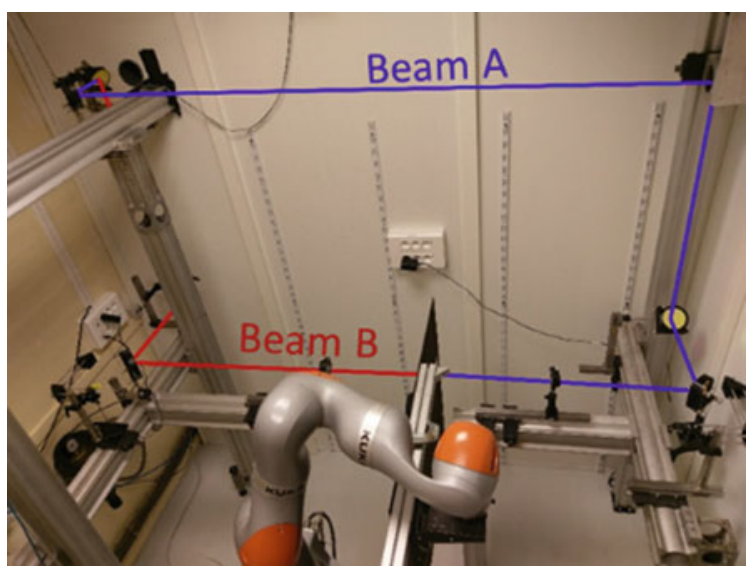


Fig. 5.32 Generated plasma pressure as a function of flux with epoxy, water, and a thick transparent PPE adhesive tape used for confinement during the LASAT investigations
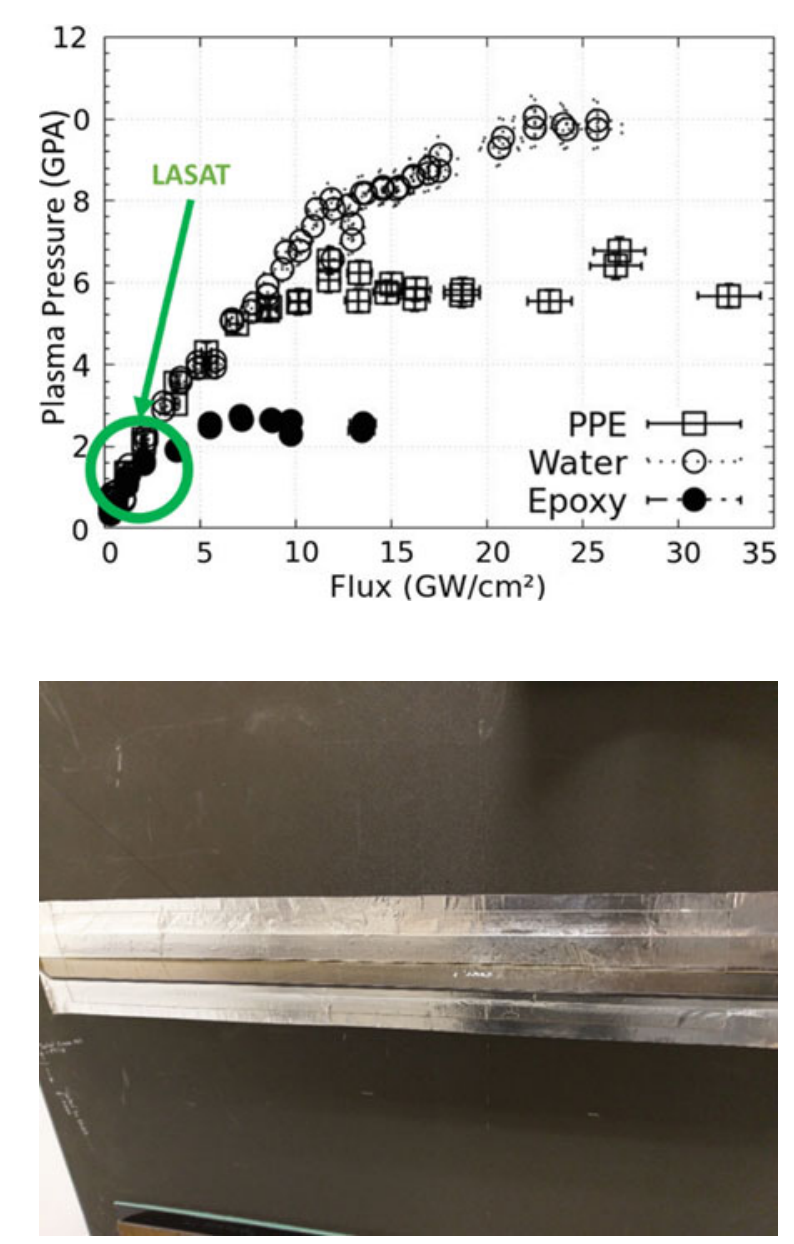

Fig. 5.33 Aluminum sacrificial layer and the adhesive tape-based confinement applied to a realistic production CFRP panel for the LASAT investigation

each calibration area, the rest of the sample was characterized by shooting at $80 \%$ of this value, regardless of the contamination state.

\section{LASAT results for the production panel}

To test the bonded CFRP panel of the production user case, the whole stiffener was spot-wise shot with an offset of $20 \mathrm{~mm}$ between consecutive shots, as presented in Fig. 5.34. This spacing was selected to avoid compromising an area with a crack that could have appeared in an adjacent tested zone.

The threshold of the bonded regions based on either the contaminated or the clean adherend surface area was isolated and a total of 53 shots were realized on one of the stiffeners. The achieved results are summarized in Table 5.5. 


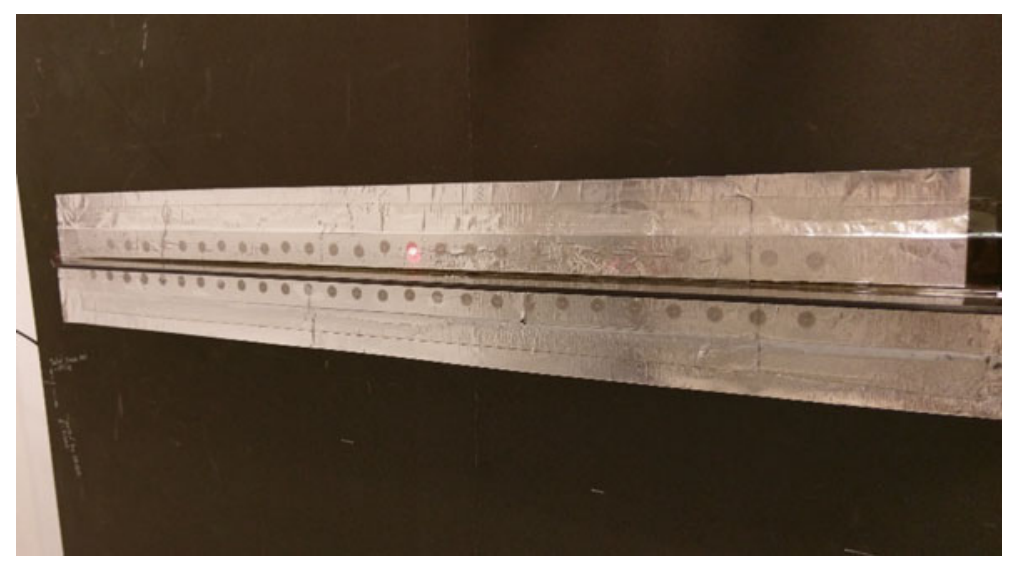

Fig. 5.34 A prepared production CFRP panel after S-LASAT investigation

Table 5.5 Summary of the LASAT results obtained in bonded regions based on either the clean (sound) or contaminated adherend surface area of the CFRP panel of the production user case

\begin{tabular}{l|l|l|l}
\hline & Threshold $\left(\mathrm{GW} / \mathrm{cm}^{2}\right)$ & Total test shots sym $80 \%$ & Opened bond sym $80 \%$ \\
\hline Sound area & 0.85 & 32 & 0 \\
\hline Contaminated area & 0.74 & 21 & 21 \\
\hline
\end{tabular}

Briefly, two distinctive opening fluxes were found. Furthermore, during the performed 53 laser shots, none of the tested areas on the sound part of the stiffener were opened. Finally, all contaminated areas failed in the test, meaning that the symmetrical LASAT (S-LASAT) results were conclusive for the production panel.

\section{Synopsis: The findings for bonding quality assessment within the production user case}

The advanced setups and workflows for the EMI, NLUS, and S-LASAT inspections used for assessing the bonding quality of the realistic bonded CFRP parts investigated in the full-scale production user case within the ComBoNDT project enabled a differentiation of bonded regions based on clean or contaminated adherend surface areas. The outcomes of the three-day demonstration event in the ComBoNDT project demonstrated the enhanced technology readiness levels of these techniques for production user cases involving the adhesive bonding of composite adherends. 


\subsection{Results of the Full-Scale Demonstration: The Representative Bonded Repair User Case of an Airbus A350 Panel}

In this section, we report on the findings achieved for the repair user case during the three-day full-scale demonstration within the ComBoNDT joint research project. First, we show the outcomes of the surface quality assessment of an Airbus A350 panel prepared for bonded repair, after which we detail the bonding quality assessment. Recalling the workflow of the repair user case, the bonding process was performed after scarfing the CFRP part to be repaired. Moreover, the intentionally deposited contamination refers to the application of DI and FP from gloved fingers coated with hydraulic oil.

\subsubsection{Surface Quality Assessment After Scarfing}

Here, we detail the results achieved with the AWT, FTIR, e-nose, LIF, and laser vibrometry inspections used for the surface quality assessment of scarfed CFRP surfaces before performing the bonded repair. In more detail, the scarfed area of a convex fuselage part was split into two semi-circles. One side was contaminated with the combined "DI-FP" contamination, while the other side was left blank.

\subsubsection{Results for AWT}

For the repair user case, we inspected a circular shaft with a defined contamination consisting of DI and FP and approximately $200 \mathrm{~mm}$ in diameter. The measurement and analysis of the repair part were conducted as follows and as depicted in Fig. 5.35.

First, the calibration of the robot for manipulating and positioning the head of the AWT measuring device was performed. The acquisition of original image data was achieved by conducting measurements of different sections in the area of interest. All the obtained images were then processed using the Convolutional Neural Network (CNN) for droplet detection. Such processing by the neural network results in the generation of heatmaps that allow an assessment of the droplet count (and the surface density of the droplets), the droplet diameter, the certainty, and the wetting ratio. At this point, the evaluated measurement results were available. The next step was classifying the local state of the surface. We achieved this material surface and process-related interpretation with a support vector machine (SVM) based on the local values of the various heatmaps. A class training was performed using a byhand delimitation of the defined surface states (namely clean and contaminated by DI or FP). The training of the SVM with the hand classification of the assessed surface states was based on $40 \%$ of the data for each class. Finally, the classification results for the entire surface were obtained through the trained support vector machine. 

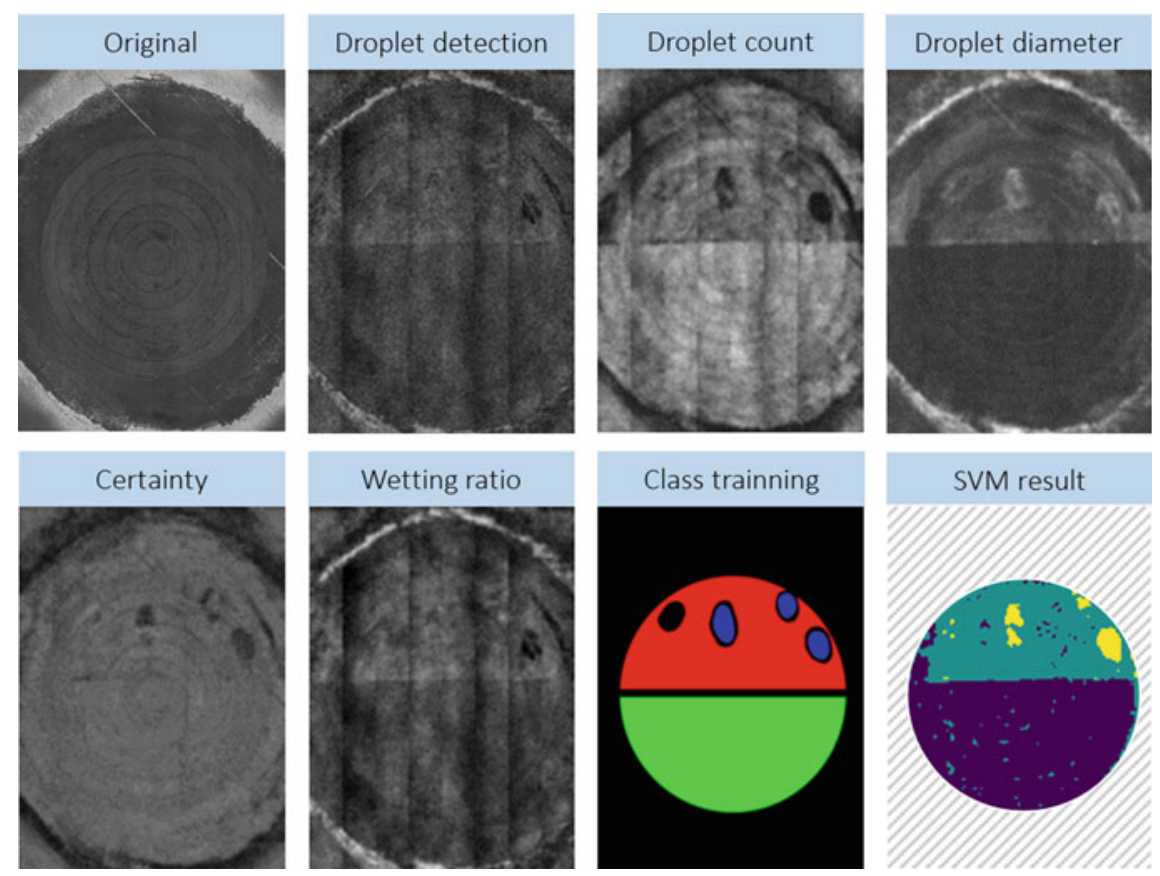

Fig. 5.35 Image-based sketch showing various steps of the AWT-related image treatment, starting from the captured image and going towards the identification of the contamination class. The offcolor final result presented in the chart labeled "SVM result" indicates clean (purple) surface regions and regions contaminated by DI (green) or FP (yellow)

As a final result, we achieved a local classification of the contamination state indicated by an off-color map. This is the output of the SVM trained with the standard local output parameters of the CNN, namely droplet diameter, wetting ratio (percentage), certainty of the neural network output, and droplet count for determining the areal density of the droplets. The findings presented in Fig. 5.36 clearly indicate that the surface regions with either type of intentionally deposited contamination can be differentiated from the clean surface regions.

\subsubsection{Results for FTIR}

To characterize the surface of the technically realistic CFRP part, FTIR spectroscopy was applied. An impression of the measuring process using the portable spectrometer is presented in Fig. 5.36. Based on the spectral findings and the application of a partial least squares (PLS) algorithm for the surface region that had been intentionally contaminated with DI, a potassium concentration in the range of 9 at. $\%$ was indicated, which is considered plausible. However, a fairly high amount of DI was detected unexpectedly on the reference side of the CFRP surface, which is some centimeters 

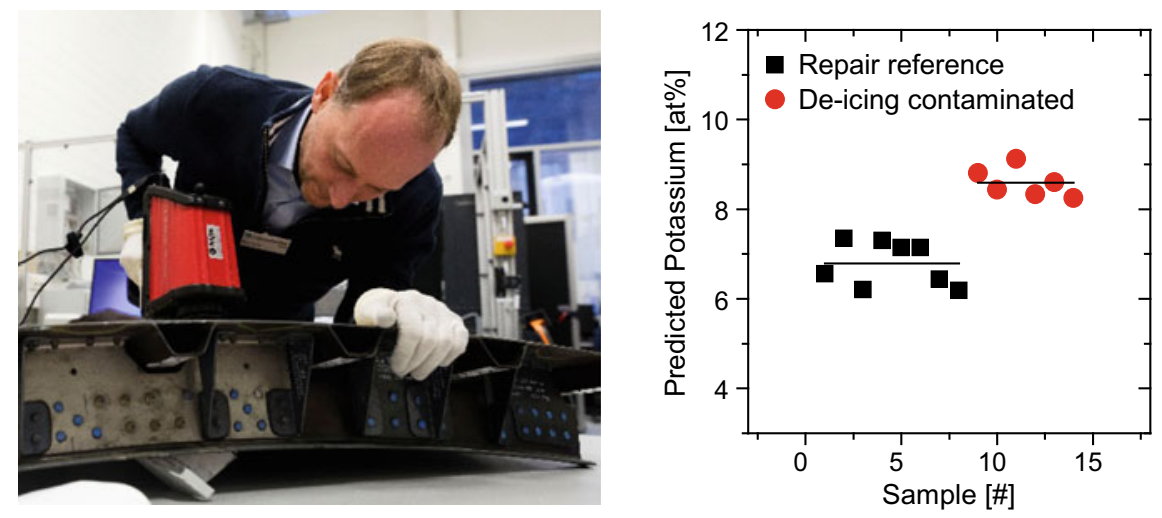

Fig. 5.36 (Left) image showing the performance of the FTIR investigations and (right) plot indicating the amount of potassium on 14 sampling regions as obtained with a PLS algorithm from FTIR spectra recorded on a technologically realistic part

away from the half of the scarfed area that had been contaminated with DI during the sample preparation. As the part was subsequently stored in an oven at elevated temperatures, we suggest that this treatment may have caused some of the DI to be transported to originally clean surface regions, e.g., following migration or after partial evaporation and subsequent condensation on the specimen surface.

\subsubsection{Results for E-nose}

During the three-day full-scale demonstration event, we tested the two distinct e-nose setups advanced in the ComBoNDT project.

\section{Airbus Group Innovations' desorber device}

Figure 5.37 illustrates how we applied the Airbus desorber device to conduct measurements on the technologically realistic parts.

Figure 5.38 presents an overview resulting from evaluating the data obtained on technologically realistic or real parts either in the production or the repair user case, benefitting from the PLS-DA model established during the testing of the pilot samples. We infer that the findings of all measurements can be separated into classes corresponding to clean or contaminated CFRP surface states, even for the complex geometries being tested. Once again, the threshold in the graph indicates the applicable parameter level differentiating surface regions that may be assigned to either class.

Performing cross-validation with the obtained, we obtain the confusion matrix of cross-validation, which is shown in Table 5.6. 

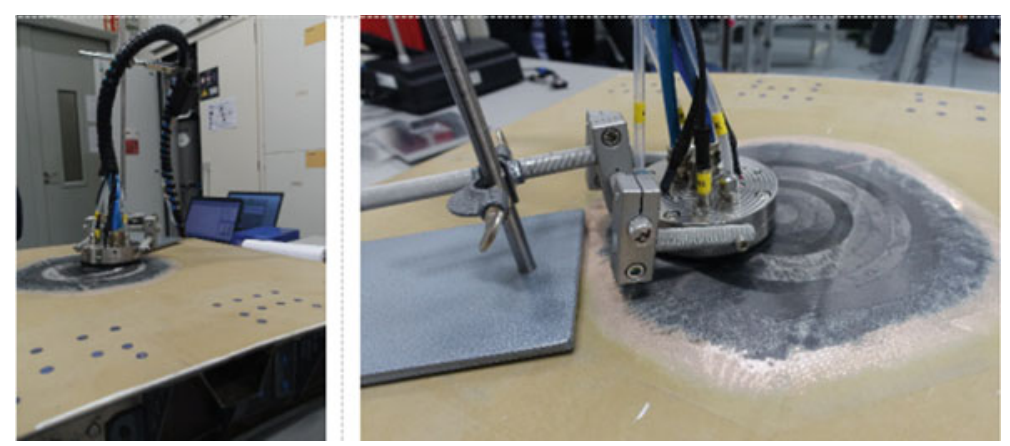

Fig. 5.37 (Left) the e-nose desorber device in use while conducting measurements on a CFRP fuselage part and (right) a close-up view of a scarfed area of a fuselage part

Fig. 5.38 Results for the class prediction of the PLSDA of data obtained from desorber device e-nose measurements on technologically realistic or real parts of the production or repair user case

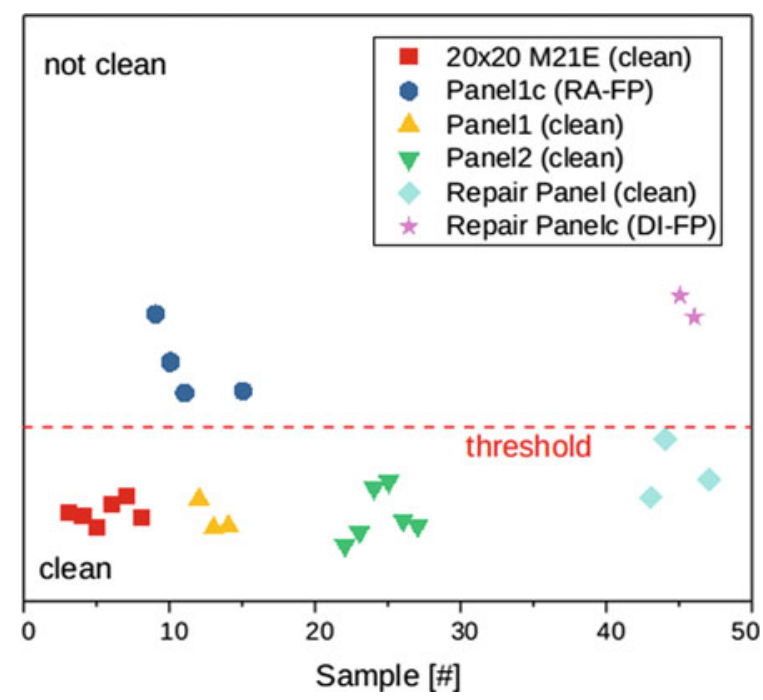

\begin{tabular}{l|l|l|l}
\hline Actual class & REF & RA-FP & DI-FP \\
\hline Predicted as REF & 18 & 0 & 0 \\
\hline Predicted as RA-FP & 0 & 4 & 0 \\
\hline Predicted as DI-FP & 0 & 0 & 2 \\
\hline
\end{tabular}

Table 5.6 Confusion matrix obtained when cross-validating the treated e-nose data shown in Fig. 5.38

\section{ENEA setup}

Using the ENEA e-nose setup, the same investigation process was performed for the repair user case as for the production user case. Specifically, six measurements were taken from areas in the clean CFRP panel region. These were used to build the ellipsoidal 2-sigma coverage detector scheme for samples contaminated following 
Table 5.7 Confusion matrix obtained when employing the ENEA e-nose for contamination detection on real composite parts from the repair user case

\begin{tabular}{l|l|l}
\hline Actual class & REF & DI-FP \\
\hline Predicted as REF & 3 & 0 \\
\hline Predicted as contaminated & 0 & 3 \\
\hline
\end{tabular}

the "DI-FP" scenario. Six measurements in the scarfed region were recorded for testing purposes: three from clean areas and three from contaminated areas.

The elicited response from the contaminated samples was significant and the contaminated samples were revealed without any false assignments. The obtained confusion matrix is presented in Table 5.7.

\subsubsection{Results for LIF}

In the final step of the research, the LIF technique was used to study the surface composition of a real aircraft part, namely a CFRP panel with a stepped area for bonded repair, as displayed in Fig. 5.39.

In Fig. 5.39, we can distinguish eight numbered circular rings that represent separate areas of the inspection. The material layer numbered 1 incorporates the outer

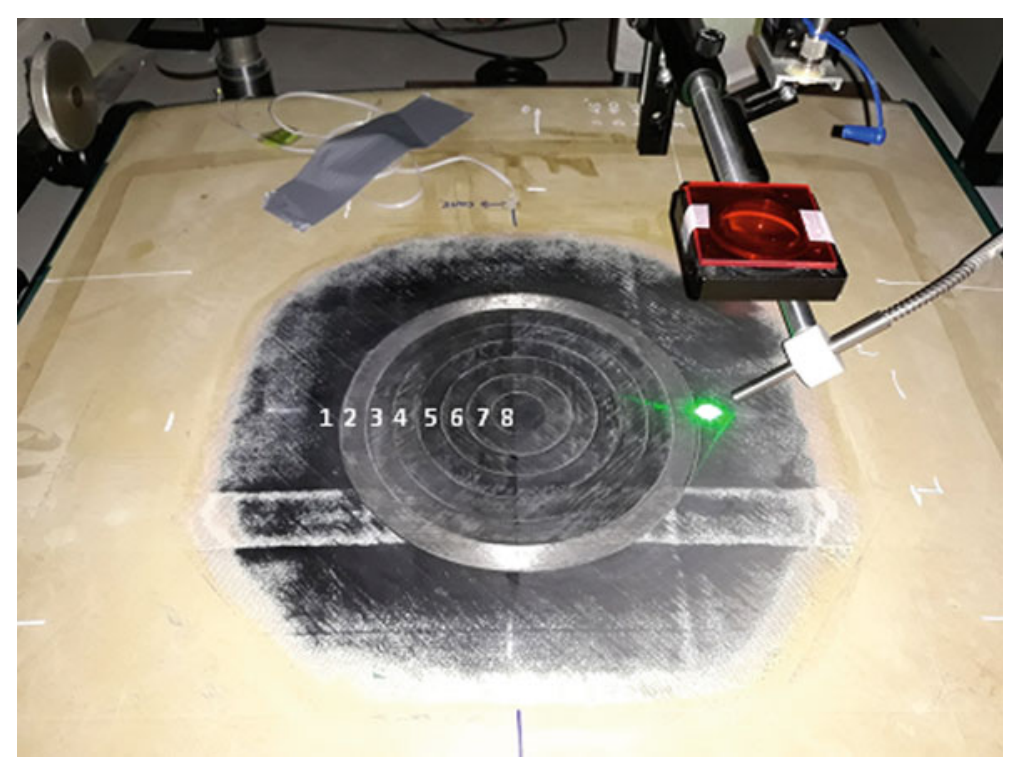

Fig. 5.39 Inspection of a real aircraft part using the LIF technique. The left semi-circular scarfed subarea appears darker and is contaminated, while the brighter right subarea is clean; the circular rings of the stepped surface prepared for bonded repair are numbered, with number 1 indicating the topmost material layer 
layer of the CFRP part without primer and copper mesh. The subsequent lower-lying seven rings (numbers 2 through 8 ) constitute the steps used (as adherends) for the bonded repair area. The left half of the specimen's surface area is contaminated. The right side is clean and provides the reference area for the LIF measurements, which were made at three points at each of the eight areas. Separate measurements were made for the clean region and the contaminated part. The obtained fluorescence intensities at each of the three points were averaged and the standard deviation was calculated. The thus obtained results are presented in the plot in Fig. 5.40. In this presentation, the blue bars correspond to clean areas, while the red bars correspond to contaminated areas. It should be noted that clean areas are characterized by higher LIF intensities, except for areas 2 and 3. In the case of areas 1 and 5, the intensity obtained in the clean subarea is almost double that of the contaminated part. For the remaining cases, the differences are less significant. In conclusion, based on these investigations, the LIF intensity did not allow the surface states to be distinguished across all of the two distinctly prepared areas of the realistic aircraft part. This could be associated with the type of contamination used ("DI-FP"). However, based on the comprehensive set of LIF inspection data acquired here, there is evidence of a significantly inhomogeneous distribution of fluorescence intensities across the scarfed surface regions.

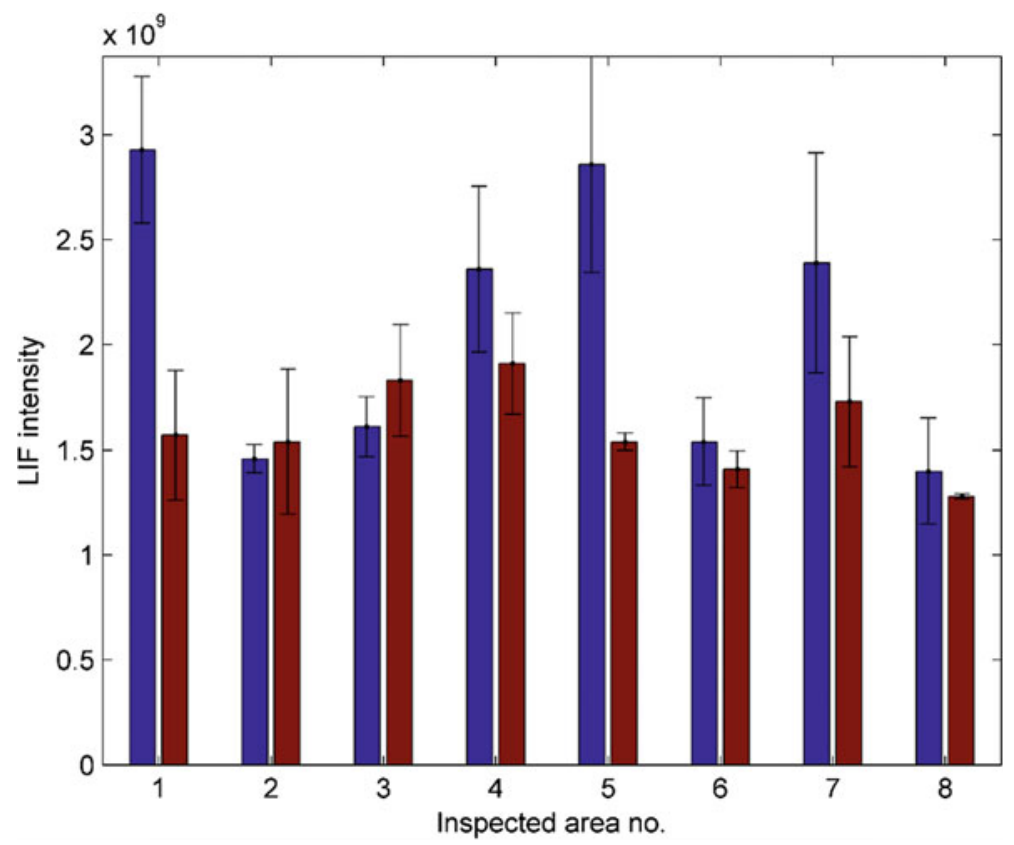

Fig. 5.40 LIF results from the inspection of a real CFRP aircraft part; the blue bars correspond to clean areas; the red bars correspond to contaminated areas; the numbers of inspected areas denote the ring zones defined in Fig. 5.39 


\subsubsection{Results for Laser Vibrometry}

In the final step of the surface quality assessment of an Airbus A350 panel prepared for bonded repair, the vibrometry technique was used to study a realistic aircraft CFRP part.

As displayed in Fig. 5.41, the specimen to be inspected had been scarfed for bonded repair, and thus, a stepped region was locally exposed. In order to excite elastic waves in the part, a piezoelectric sensor was mounted close to the surface region of interest. As can be seen in Fig. 5.41, the disc-shaped piezo element was located on the panel surface area covered with primer, meaning it was some centimeters away from the grinded area (where the black CFRP is contrasted in Fig. 5.41) that surrounds the mechanically treated stepped region prepared for bonded repair. Again, we can distinguish eight (height) levels within the inspection area. The first is the CFRP without primer and copper mesh and the next seven levels correspond to the surfaces of the steps introduced by scarfing. The right half of the blackish area was intentionally contaminated. The left side was left clean and the surface areas in this region served as references for the inspection. As shown in Fig. 5.41, the piezoelectric disc was placed alongside the borderline between the two areas in a somewhat symmetrical position so that the elastic wave could propagate in both areas (the clean and contaminated halves of the surface) in the same way. The spatially resolved plot for the root mean square (RMS) of the vibration energy as obtained from the vibrometer software is depicted in Fig. 5.42. This is overlaid on the top view photograph of the inspected region of the CFRP part. As in Fig. 5.41, the contaminated area is on the right side. The total area prepared for bonding is located between two omega stringers, as may be perceived in the foreground of Fig. 5.41. The excitation of an elastic wave in this area results in concentrations of the wave energy between the stringers, which manifest as vertical lines in Fig. 5.42. Moreover, the central image section of the displayed RMS plot shows a circular pattern corresponding to the stepped area on the specimen's surface. The data processing used for this part followed the whole area scan employed for the moisture-enriched samples and pilot

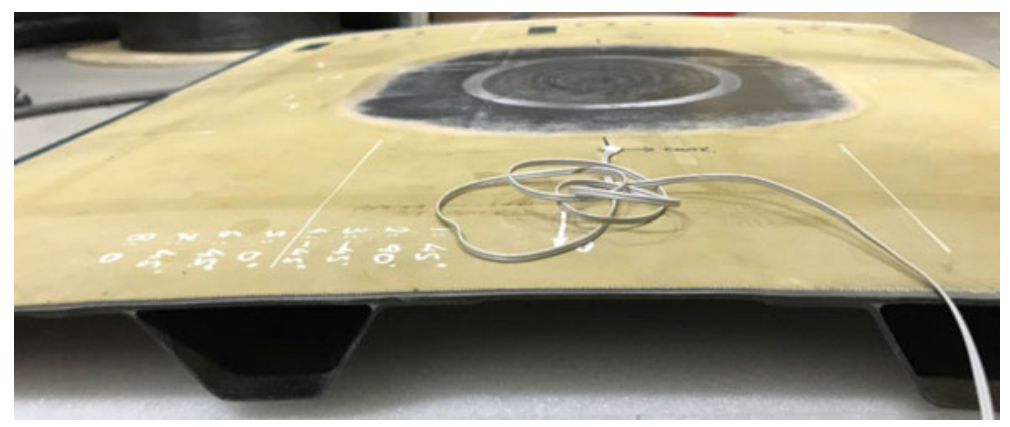

Fig. 5.41 The setup for the laser vibrometry inspection of a real CFRP panel with the scarfed and stepped areas for bonded repair 


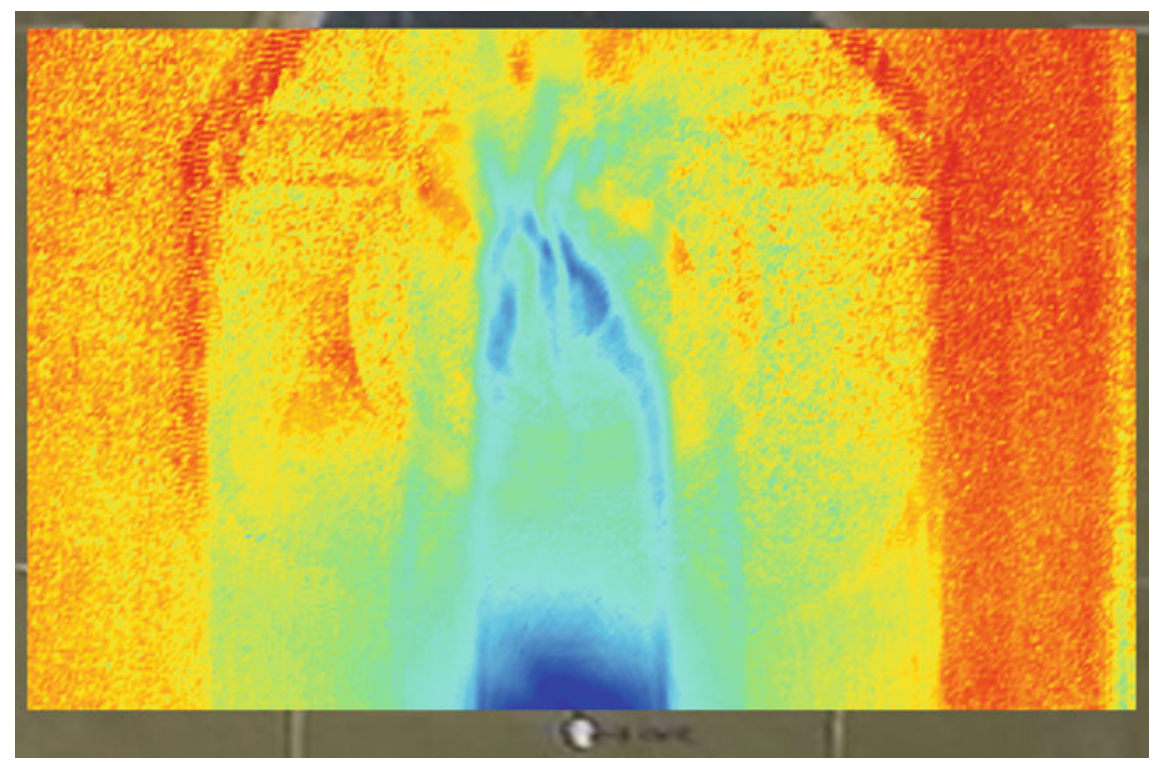

Fig. 5.42 Space-resolved RMS plot obtained from the vibrometer software. The image section is roughly half of that shown in this figure. As illustrated by the lateral view, the roundish delimitation of the area prepared for bonding is located between two omega stringers. Excitation of an elastic wave in this area results in concentrations of the wave energy between the stringers, as indicated by greenish and blueish colors

level samples. Specifically, only the data points corresponding to the stepped area were considered here. Next, this set of data points was divided into two halves. The data points obtained in the surface region shown on the left half represented the referential case, and by processing them, the $\mathrm{E}$ (proportional to vibration energy) and $3 \sigma$ values were calculated. The same procedure was conducted for data points from the right stepped half of the panel surface. The clean area is characterized by the values $\mathrm{E}=3.58 * 10^{-7} V^{2}, 3 \sigma=9.96 * 10^{-7} V^{2}$, while the contaminated area showed $\mathrm{E}=3.57 * 10^{-7} V^{2}, 3 \sigma=9.68 * 10^{-7} V^{2}$. We discern that the $E$ values are practically identical and that there is a slight difference in the $3 \sigma$ values. We conclude that these parameters do not allow the two areas of the realistic aircraft part to be distinguished, although the state of half the panel's surface could be associated with the application of the contamination used in the "DI-FP" contamination scenario.

\section{Synopsis: Findings for the surface quality assessment within the repair user case}

The challenge of the ENDT-based surface quality assessment consisted of identifying intentionally deposited combined DI and FP contamination on a scarfed real Airbus A350 CFRP panel. The surface area in contact with DI was more than one order of magnitude larger than in the case of FP. Moreover, indications were obtained that the surface area affected by potassium-containing substances from the DI expanded after 
the panel was oven-heated at elevated temperatures. Depending on the assessable area for each inspection method, the level of detail of the gained insights was different.

The findings achieved with the AWT clearly indicate that surface regions with either type of intentionally deposited contamination can be differentiated from clean surface regions.

The results obtained when we applied the enhanced e-nose techniques make us confident of the accuracy achieved with these devices for detecting "RA-FP" or "DI-FP" mixed contaminations at the presented levels of contamination, both in the production and repair user cases and with realistic and real parts.

Comprehensive LIF inspection data provided evidence of a significantly inhomogeneous distribution of fluorescence intensities across the scarfed surface regions, but the evaluation of the data subsets did not enable a reliable local differentiation between clean and contaminated regions. Based on the FTIR spectroscopic outcomes obtained with a portable spectrometer and the subsequent application of a partial least squares (PLS) algorithm, indications for an intentional deposition of DI were successfully substantiated.

Given the ample specimen geometry and the expectable substantial expenditure of time, the stepped surface area of the scarfed and stepped CFRP specimen was not investigated by laboratory-based reference analysis for reviewing the actually applied contamination degree.

As indications displayed by AWT and other inspection methods like FTIR differ with respect to the extent of DI-related contaminations, we recommend to utilize the presented enhanced ENDT approaches in future projects in order to perform additional tests for validating the data post-processing, e.g. by applying a PLS algorithm, when inspecting realistic parts.

\subsubsection{Bonded Repair Assessment}

Subsequently, we report on the outcomes obtained using the EMI, NLUS, and LASAT inspections conducted for the bonding quality assessment after performing the bonded repairs.

\subsubsection{Results for EMI}

In Fig. 5.43, we present a photograph of a real aircraft CFRP panel with a tailored and adhesively bonded circular repair patch. The two piezoelectric sensors attached to the top of the patched area are also visible. On the left, the sensor labeled DD5 is positioned over the clean bonding area, while the second sensor (DD8) shown on the right is placed over the contaminated region. Both sensors were attached in a gluing process using a cyanoacrylate adhesive.

The selected electromechanical impedance characteristics of the sensors where measured two times. First, the free sensors were characterized before gluing for 


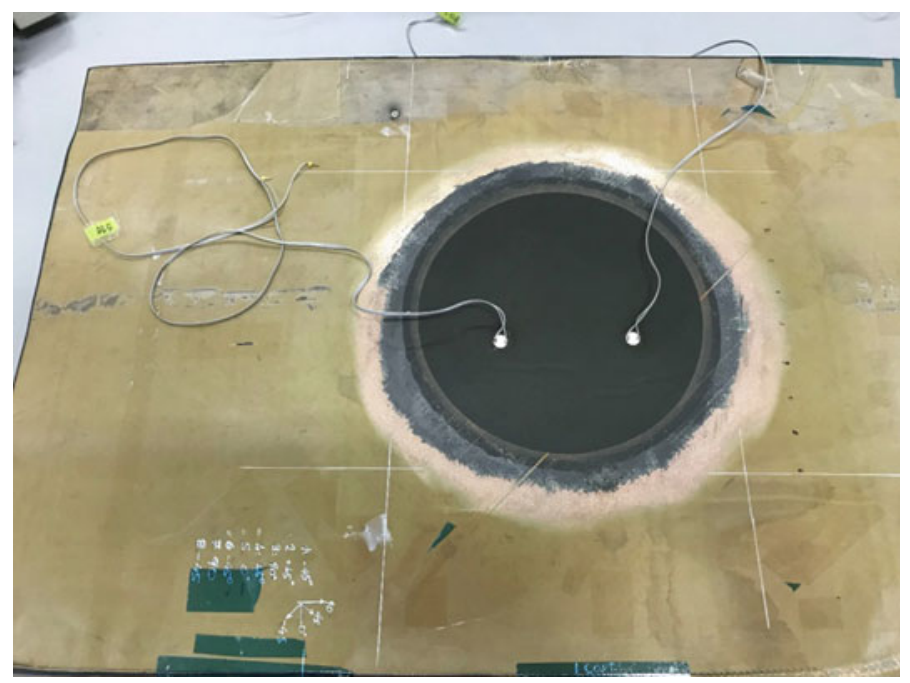

Fig. 5.43 Photograph showing a real aircraft CFRP panel with an adhesively bonded circular repair patch

referential purposes. Second, the signals from the bonded sensors were acquired onsite while the panel was lying freely on the laboratory table and in an arrangement similar to the one displayed in Fig. 5.43.

When we investigated the realistic part after the bonded repair for the repair user case, we did not observe any additional local resonances using the sensors. This is in contrast to the observations made for the production panel that was structured in a significantly different way. The results of the conductance $(\mathrm{G})$ analysis are depicted in Fig. 5.44a. It can be seen that gluing the sensors causes the RMS values to drop.
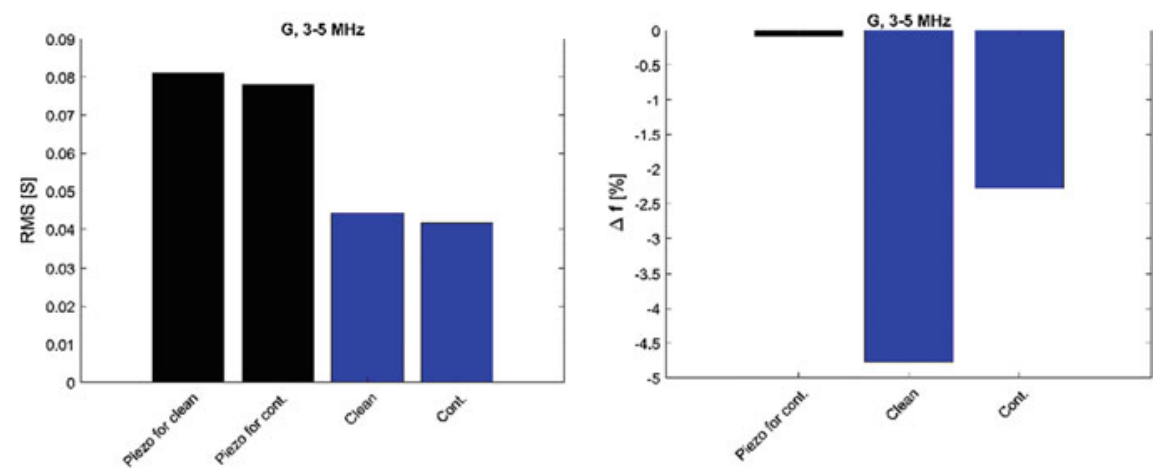

Fig. 5.44 EMI results in the 3-5 MHz frequency region for the six joint states of a realistic CFRP aircraft part that was repaired and investigated within the repair user case; a RMS values of conductance and $\mathbf{b}$ frequency shift of the conductance peak 

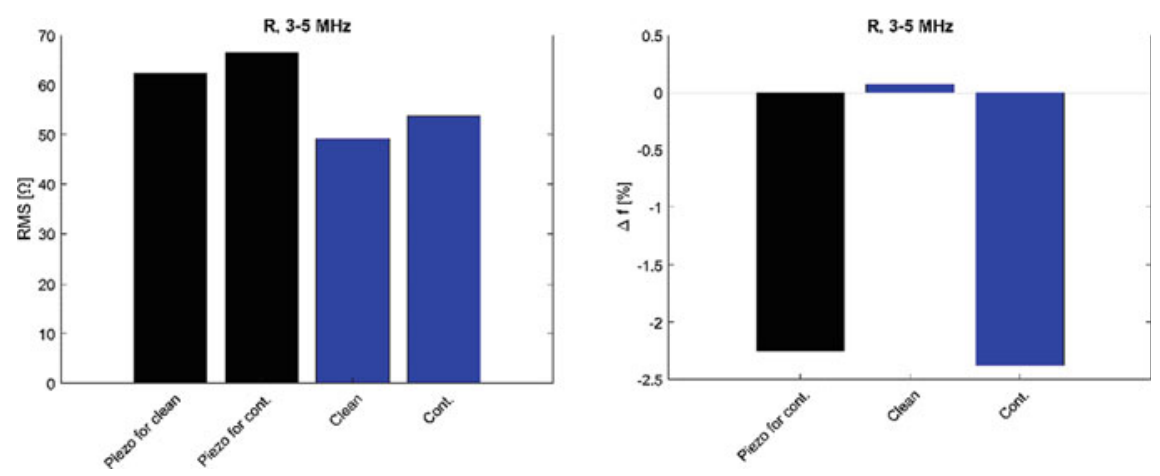

Fig. 5.45 EMI results for 3-5 MHz frequency region for six investigated states of a realistic part from the repair user case; a RMS values of resistance presented and $\mathbf{b}$ frequency shift of the resistance peak

This finding indicates that the sensor vibrations have a lower amplitude, which is attributed to damping effects. The findings for the sensor over the clean region were quite similar to those for the one over the contaminated region. In Fig. 5.44b, we present the measured frequency shift of the conductance maximum. The respective shift for the sensor positioned over the clean bonding region was more than double for the sensor glued over the contaminated region. So, the frequency shift indicates a significant change in comparison to the behavior of the RMS values.

Next, we investigated the resistance curves in the same frequency range (3$5 \mathrm{MHz}$ ). The gluing of the sensor resulted in a drop in the respective RMS values (Fig. 5.45a). But the drop was not so significant as for the conductance. Interestingly, we observe that the differences between the RMS values of the two sensors are almost the same for the two investigated sensor states, i.e., the free sensor state and the glued sensor state. The values for the respective differences between the two sensors were found to be 4.25 and $4.53 \Omega$, respectively. Also, the frequency shift did not differ significantly between these two states (Fig. 5.45b), since almost the same value of shift is observed between the two free sensors and between the free sensors and sensor bonded to the contaminated bond area. The results for the resistance are interpreted as a low sensitivity of the high-frequency region of resistance to the supposed structural differences between the clean and intentionally contaminated bonding regions in the inspected part.

\subsubsection{Results for NLUS}

The fundamental frequency field obtained through the NLUS inspection of the real aircraft CFRP panel of the repair user case with the bonded circular patch is visualized in Fig. 5.46, and it shows a rather conventional standing wave pattern over the whole area of the repaired region. In contrast, the higher harmonic fields measured in the patch region (shown in Fig. 5.47) demonstrate quite different nonlinear field 


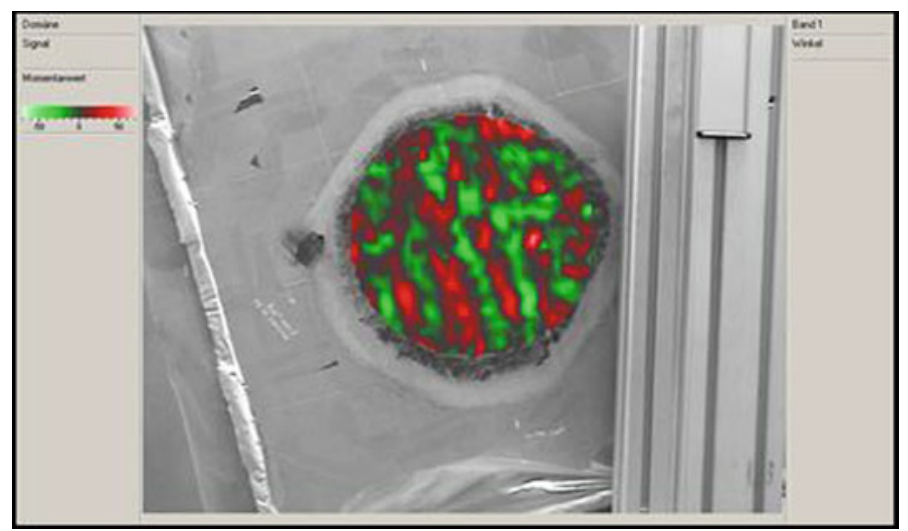

Fig. 5.46 NLUS fundamental frequency field excited in the bonded repair patch region of the CFRP specimen from the repair user case

distributions. The second and third harmonics presented at the top and bottom of Fig. 5.47 reveal a strong enhancement of the nonlinearity and, therefore, indicate a lower bonding quality in regions of the patch that are discernible at the right-hand side and lower part of the patch.

To reiterate here, we also observed an enhancement of nonlinearity in joint regions with weaker bonds when we were mapping and imaging bonded joints based on locally contaminated adherends in realistic aircraft components. In both user cases, the accuracy of the accordance between the positions of the intentionally prepared areas with weakened bonds and the regions identified and visualized with the NLUS approach was confirmed by the respective manufacturers of the contaminated specimens.

\subsubsection{Results for LASAT}

In view of the challenges of a LASAT inspection, the repaired CFRP panel of the repair user case comprised an adhesive bond at different depths, depending on the positioning of the tested subzone with respect to the region of the repair patch. In consideration of this fact, we defined a specific time delay for each inspected subzone of the bond to set the maximum tensile stress induced during a LASAT shot on top of the respective bond location. Moreover, the CFRP panel featured stiffeners on one side. Therefore, we modified and adapted the testing procedure since access to both sides of the panel was not viable for every shot.

Figure 5.48 presents a sketch diagram showing the selected shot pattern as well as the general geometry of the panel.

The panel is partitioned into three distinct main areas, namely the stiffener area (highlighted in dark brown in Fig. 5.48 where the panel can only be accessed from one side, the stiffener flanges (lighter brown), where the stiffener is bonded to the 

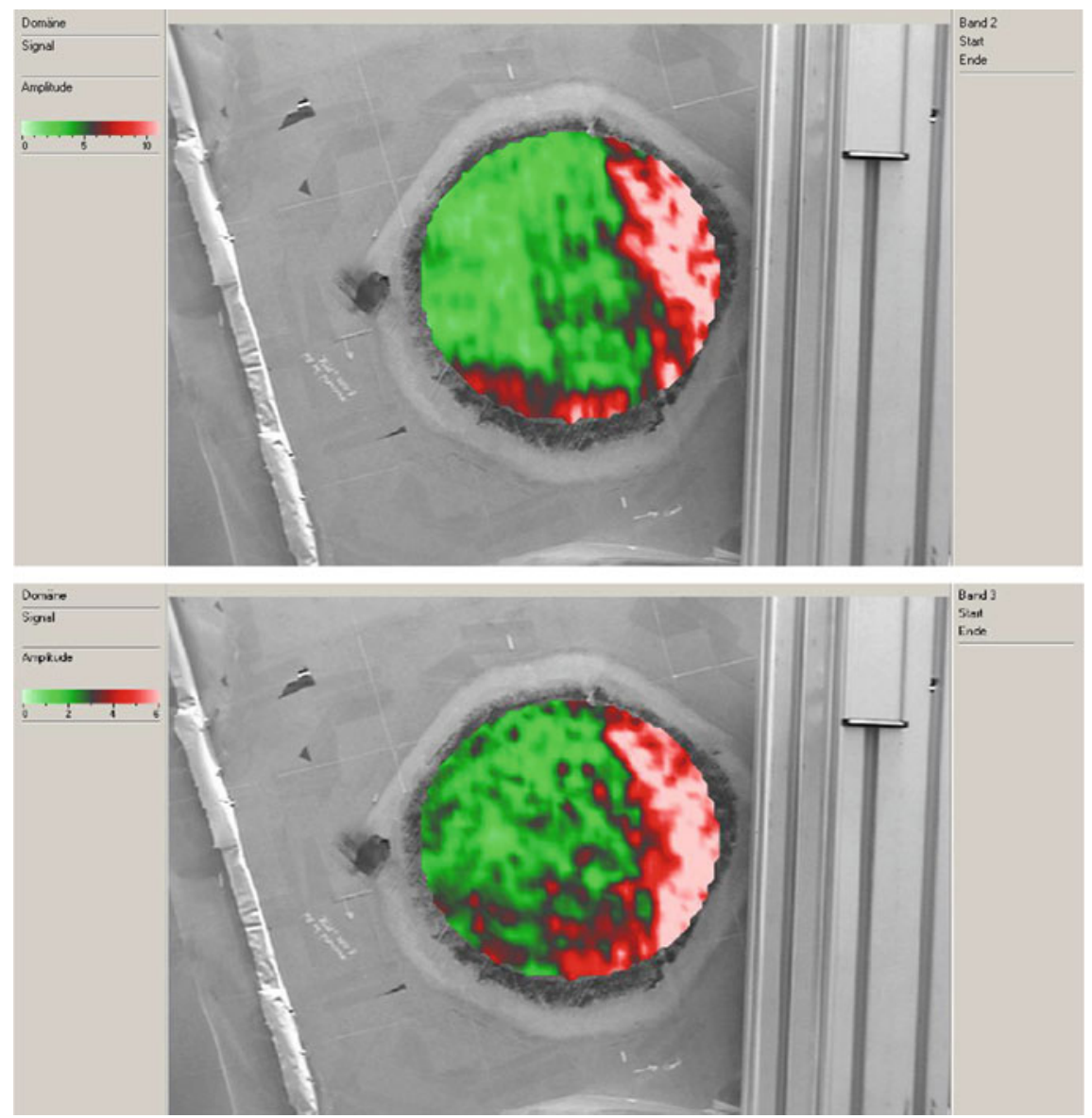

Fig. 5.47 The second harmonic ( $40 \mathrm{kHz}$, top) and the third harmonic $(60 \mathrm{kHz}$, bottom) NLUS fields in the repair patch of the fuselage panel from the repair user case

panel, and the actual panel (light brown). As the whole panel was tested, S-LASAT was complemented by standard mono-shot LASAT in the stiffener area. The obtained results are summarized in Table 5.8.

Without applying the enhanced LASAT technique, we were unable to achieve a clear differentiation between a contaminated and a sound region of the patch on top of a stiffener. Both thresholds were alike, and from the ten LASAT shots performed on the contaminated area, only two showed indicators characteristic of an opening. The comparatively low energy required to open the bond might be explained by the actual state of the repaired panel. Numerous porosities were found by the ultrasonic test performed after the repair. Following the line of action for a quality assurance procedure of a repair process, this finding would be considered an indication that the 


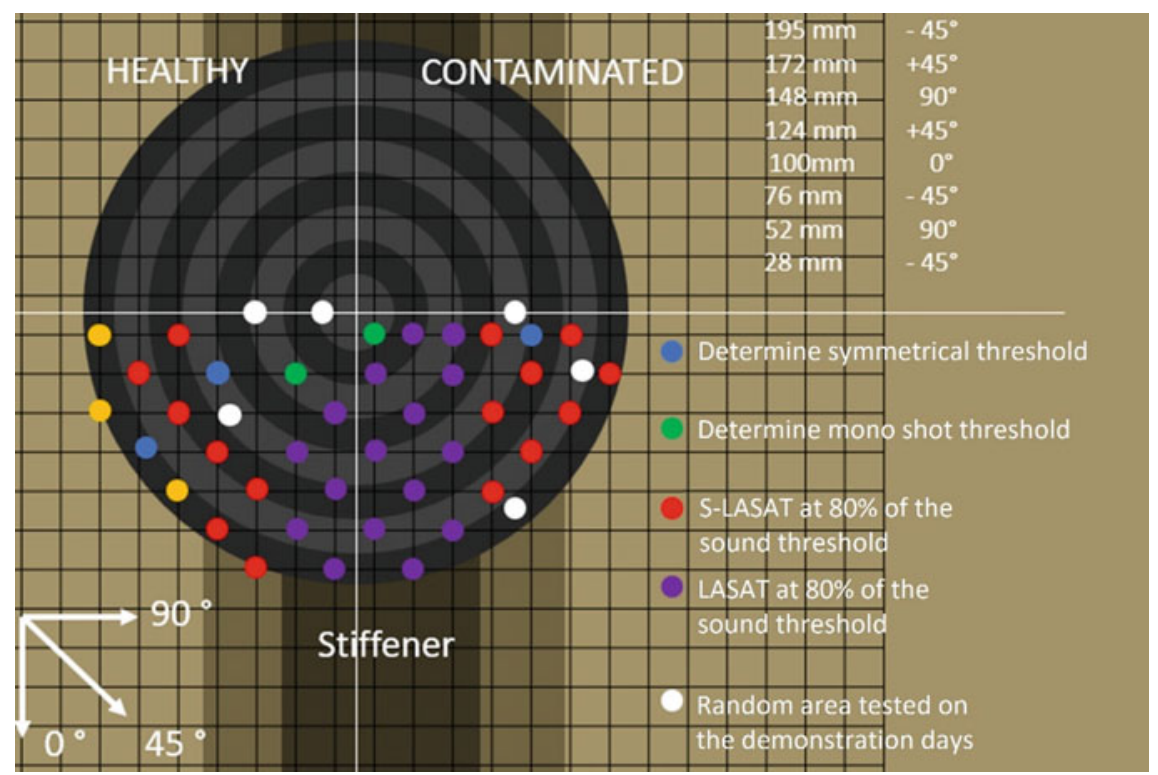

Fig. 5.48 Diagram of the LASAT shot partitioning on the adhesively bonded repair patch within the CFRP panel of the repair user case. Contaminated joint regions (in contrast to clean ones) are based on intentionally contaminated adherend surface regions

Table 5.8 Summary of the LASAT results obtained for the repaired CFRP panel of the repair user case for sound and contaminated regions of the repair patch

\begin{tabular}{l|l|l|l|l|l|l}
\hline & $\begin{array}{l}\text { Threshold } \\
\text { S-LASAT } \\
\left(\mathrm{GW} / \mathrm{cm}^{2}\right)\end{array}$ & $\begin{array}{l}\text { Total test } \\
\text { shots } \\
\text { mono 80\% }\end{array}$ & $\begin{array}{l}\text { Opened bond } \\
\text { mono 80\% }\end{array}$ & $\begin{array}{l}\text { Threshold } \\
\text { S-LASAT } \\
\left(\mathrm{GW} / \mathrm{cm}^{2}\right)\end{array}$ & $\begin{array}{l}\text { Total } \\
\text { test } \\
\text { shots } \\
\text { sym } \\
80 \%\end{array}$ & $\begin{array}{l}\text { Opened } \\
\text { bond sym } \\
80 \%\end{array}$ \\
\hline Sound area & 0.63 & 7 & 0 & 0.72 & 8 & 0 \\
\hline $\begin{array}{l}\text { Contaminated } \\
\text { area }\end{array}$ & 0.63 & 10 & 2 & 0.61 & 8 & 8 \\
\hline
\end{tabular}

workers did not perform a high-grade repair task. During the full-scale demonstration, we performed further inspection. Although the state of the panel did not meet the demands, we achieved proper discrimination between the repair region on the contaminated adherend surface area and the one on the sound adherend surface by applying S-LASAT. We observed that only the area tested on the contaminated part failed the LASAT test, and thus it was identified.

Advancing the S-LASAT technique enabled us to differentiate, for both inspected panels, the contaminated area from the sound one. We faced some limitations of the basic LASAT technique in assessing inspection regions with complex geometries: If one side of the CFRP panel was not accessible, the technique could not 
be applied and a non-optimized testing approach, such as the mono-shot setup, performed inadequately.

We consider the LASAT double-shock setup highly promising for overcoming the existing limitations in the case of complex panel shapes and we will be ready to perform further challenging studies in this vein.

\subsection{First Evaluation of ENDT Procedures Introducing a Probability of Detection Approach}

\subsubsection{Introduction and Motivation}

In this section, we conduct in-depth evaluations based on a probability of detection (POD) approach to the findings achieved with the distinct enhanced ENDT procedures. To this effect, we introduce the POD methodology and demonstrate its aptitude for selected applications in several of the user cases introduced above. In more detail, we propose cutting-edge evaluations highlighting initiatory POD for the ENDT procedures advanced in the ComBoNDT project [1] and we base these on selected findings from the previously described intentionally contaminated CFRP adherend surfaces from distinct user cases. To scale the expectations, we consider such investigations a prerequisite to any certification step. Indeed, performing a POD evaluation is mandatory for any new non-destructive testing technique deployed in service, including our applications. The subsequently described examinations were performed as an important forward-looking contribution of the ComBoNDT project to initiate prospective in-depth approaches for selecting technically appropriate ENDT methodologies and procedures for specific quality assurance tasks following a POD evaluation.

We are aware that we are unable to present a complete POD evaluation that complies with technical standards. For example, a first aspect that we consider is that none of the presented ENDT procedures is mature enough to have a fixed testing workflow with a well-defined threshold value that allows surface states to be robustly identified as "ready to bond" based on the extensive (accelerated) testing of the respectively manufactured adhesive joints. Therefore, we are as yet unable to define an exact POD procedure for evaluating ENDT procedures. For this reason, we use POD as applied for conventional NDT as a guideline, which allows us to outline the background and basic principles of such an evaluation. We repeatedly highlight in this report that there are some constraints on the formal resilience of the used data. A second argument is that real POD is very time-consuming and expensive-with good cause as it requires a large amount of reproducibly prepared specimens with representative defect types and sizes that are intentionally introduced within a representative range. However, we here merely include the three contamination scenarios that were available for our demonstration. Third, it would be necessary to involve various operators in order to account for the effects of individual human factors. In 
addition, to ensure a formal evaluation approach, the operators should not be scientists with expertise in the evaluated ENDT technique but rather adequately trained operators, and to enable blind testing, they should not have prior knowledge of the defect introduced into the samples. Clearly, for the data gathered in the ComBoNDT research project, these conditions were not fulfilled.

In spite of these differences, we consider the promising quality of the acquired data to be interesting enough to start a simplified investigation. Therefore, we propose a first POD evaluation here, albeit not for all the ENDT techniques and procedures applied and not for every contamination scenario. Rather, we advantageously select already available procedures and datasets that appear conveniently close enough to typical POD procedures.

Subsequently, in the next section, we first provide general information about POD. Then, we describe the selection and inquiry processes applied for gathering suitable datasets from the ComBoNDT project partners for POD calculations. In this context, we also explain some choices, especially regarding the values for the requested contamination levels within a distinct scenario. We present the thus obtained results in the main part of this section, whereby they are grouped by ENDT technique with no specific prioritization. In the last part of this section, we draw some preliminary conclusions and propose a synopsis table on the POD quantification to summarize the main results obtained in this work.

\subsubsection{General Information on POD Procedures and Tools}

Following our layout, a POD procedure is applicable for a given list of quantifiable features related to instantiated (sub)sets based on:

- a given material/structure to be inspected

- a given defect type

- a given NDT technique

- a given testing procedure

Regarding the user cases and scenarios from the ComBoNDT project, this means that each POD calculation is valid for one specimen type (i.e., contaminated CFRP for surface quality assessment, and assemblies comprising adhesive joints based on contaminated adherends for bonding quality assessment), one contamination scenario, and one ENDT technique and its associated diagnostic procedure. If one of these instantiations or elements changes, then the actual POD approach might change.

We designed the POD approach following the targeted purpose of accounting for the uncertainties in the capability of an ENDT technique to detect a given defect. Regarding the POD procedure for non-destructive examination (NDE), "The POD $(a)$ function is defined as the proportion of all cracks of size that will be detected in a particular application of an NDE system" [2]. The NDT result is made nondeterministic because of the potentially numerous uncertainties. For example, the actual defect (e.g., fatigue cracks), procedure, material, mechanical repeatability, 
and human factor will influence the signal response of an NDT inspection. Therefore, a POD evaluation is a statistical estimation of the performance of an NDT with a consideration of the uncertainties, accounted for via the dataset. The POD estimation uses a sample set to gather data and is thus rather more "sample-dependent" than inherently representative of the basic population.

In practice, there are two main approaches to estimating a POD, namely the hit/miss model and the signal/response model. Some key elements are provided below, and more details can be found in the literature, such as Berens [2, 3], and other publications related to non-destructive inspection (NDI) procedures, e.g., ultrasonic inspection [4] and scheduling NDI [5].

\section{Hit/miss approach}

The hit/miss approach refers to a binary diagnosis providing as its output a logical variable with the feasible values "detected" or "not detected". For each flaw size $a$ of the dataset, the "observed POD" $\operatorname{POD}_{\text {obs }}(a)$ is calculated as shown in Eq. (5.1). The POD function for all $a$ is then obtained through a parametric regression on the $\mathrm{POD}_{\mathrm{obs}}$ values. The Berens parametric regression model uses cumulative normal or log-normal functions.

$$
\operatorname{POD}_{\text {obs }}(a)=\frac{\text { Number of Hits }(a)}{\text { Number of trials }(a)}
$$

It is generally recommended to have a minimum of 60 flawed inspection sites with at least three operators.

\section{Signal/response approach}

In this approach, the signal response $\mathrm{S}(a)$ from the defect with the flaw size $a$ is used to give a quantified diagnostic with regard to a decision threshold value $s$ th $(\mathrm{S}(a)>$ $s$ th or $\mathrm{S}(a)<s$ th). For a given defect size $a, \mathrm{~S}(a)$ is considered a normally distributed random variable. The probability density function of the signal value, $\mathrm{g}_{a}$, has a mean value $\mu_{a}$ and a standard deviation $\varepsilon$ independent from $a$ (see Eq. 5.2). Then, the POD for flaw size $a$ is defined as in Eq. (5.3)

$$
\begin{gathered}
\mathrm{S}(a)=\varphi(a)+\varepsilon a(\mathrm{M})=\mu_{a}+\varepsilon \\
\operatorname{POD}(a)=\int_{\text {sth }}^{\infty} \mathrm{g}_{a}(\mathrm{~s}) \mathrm{ds}
\end{gathered}
$$

Some hypotheses must hereby be verified:

$\ln (\mathrm{S})$ and $\ln (a)$ (or $\mathrm{S}$ and $a$ ) are linearly linked: $\ln (\mathrm{S})=\beta_{0}+\beta_{1} \ln (a)+\delta$, with $\beta_{0}$ and $\beta_{1}$ representing regression parameters and $\delta$ being a normally distributed variable with zero mean and variance $\sigma_{\delta} ; \sigma_{\delta}$ is independent of $a$. 
Then, the POD function is a cumulative $\log$-normal $\left(\mu, \sigma^{2}\right)$ distribution function, with parameters shown in Eq. (5.4). The POD curve is then evaluated to estimate the $\beta_{0}, \beta_{1}$, and $\sigma_{\delta}$ that best fit the signal data.

$$
\left\{\begin{array}{l}
\mu=\left(\ln (s \text { th })-\beta_{0}\right) / \beta_{1} \\
\sigma=\sigma_{\delta} / \beta_{1}
\end{array}\right.
$$

It is recommended to have a minimum of 40 flawed inspection sites with at least three operators.

Regardless of the approach, the estimation of POD-based on several datasets for the same measuring problem leads to several POD curves and facilitates the evaluation of a confidence interval that is the dispersion in the POD results for a given amount of data obtained for the sample:

- For a given flaw size $a$, the probability of detection with $95 \%$ confidence, labeled "PODa/95", is to be understood such that the estimated probability of detection "PODa" has a 95\% chance of being higher than PODa/95;

- For a given POD, the flaw size detectable with $95 \%$ confidence, labeled " $a \mathrm{POD} / 95$ ", is such that the estimated flaw size " $a \mathrm{POD}$ " has a $95 \%$ chance of being lower than $a \mathrm{POD} / 95$;

- Typically, the most common value is POD90/95, labeled $a 90 / 95$ in this report. For a given flaw size or contamination degree, this means a probability of detection of $90 \%$ with a $95 \%$ confidence. As this value is the one generally communicated to the authorities for aeronautics applications, we selected this one in this work.

To separate and classify the measured analysis data, one important parameter is the detection threshold, and its determination can strongly influence the POD. We recommend defining it in a way that ensures that the defect signature is identified and differentiated from the noise and the saturation signal. In the case of the subsequently evaluated data obtained in the ComBoNDT research project, a saturation of the signal detection systems was not reached, and we consider the noise to be according to the signal in the case of the reference noise level was defined considering the signal on reference specimen. Therefore, we adjust the detection threshold so that it is, on the one hand, superior to the reference values obtained using cleaned adherend surfaces and, on the other hand, inferior to the lowest contamination values that could be differentiated from the reference values.

\section{POD model implementation in NDT software}

The model described above uses CIVA software, which is one of the main simulation software packages for NDT modeling.

In the upcoming section, which reports the results for selected example ENDT investigations, we always display the POD findings using the same layout, which is composed of three windows, as shown in Fig. 5.49. First, the top-left window represents the material-related data points as obtained with the respective techniques in the ComBoNDT project. The underlying system of coordinates shows the contamination 


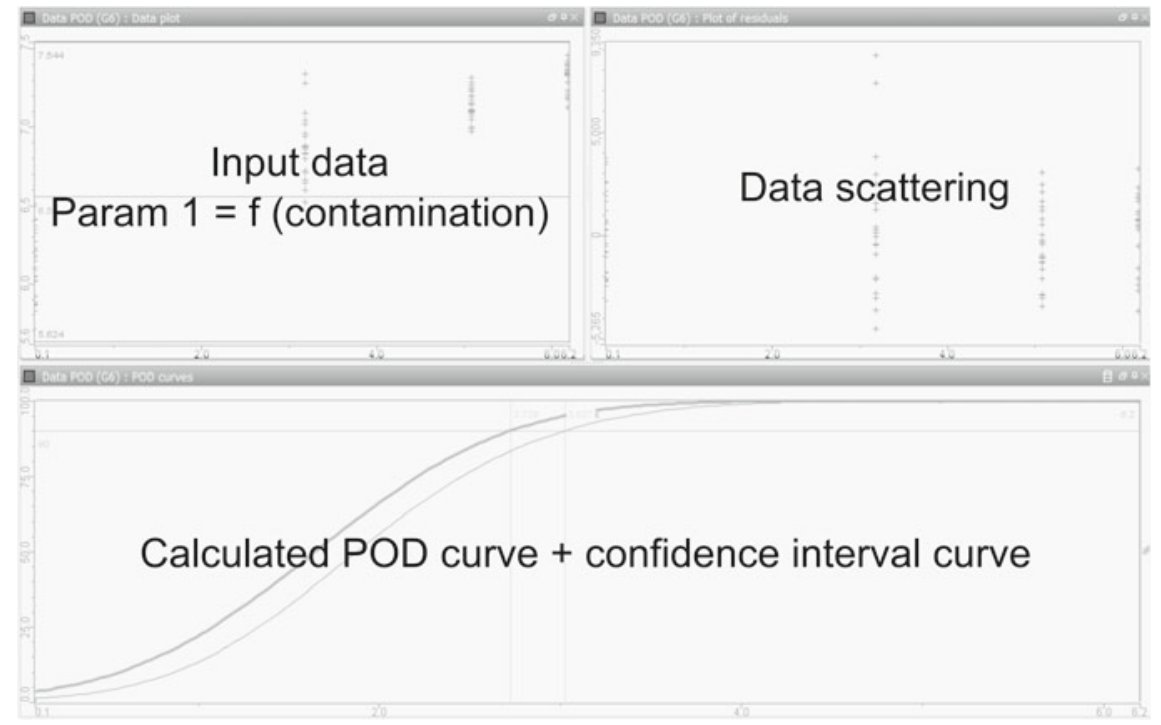

Fig. 5.49 The graphical user interface provided by the CIVA simulation software package for NDT modeling. Most importantly, the upper-left window shows the measured material-related input data and the lower window displays the calculated POD curves and the probability of detection $a 90 / 95$, as introduced in the text

values along the abscissa, as determined using a laboratory-based reference analysis. Meanwhile, the values for the feature being quantified based on the ENDT inspection are displayed along the ordinate. The dimensions and characteristics are individual for each technique and they might be, for example, amplitude values. We used the graphical user interface of this chart to adjust the detection threshold. Second, the top-right window displays the data scattering and a control parameter for the numerical model hypothesis, which is not detailed. Finally, the bottom window depicts the calculated POD curves, upon which the introduced probability of detection $a 90 / 95$ is generally displayed.

\subsubsection{Input Data for POD Calculation and Compiled Hypotheses}

For the POD calculations, we gathered example datasets acquired with advanced ENDT techniques and procedures for distinct and intentionally applied contamination scenarios within different user cases. As indicated above, these datasets were originally not measured following established POD approaches and instead may be considered instances of a challenging supplementary blind testing. In detail, we considered raw data because we wanted to maintain the statistical distribution 
within the data, and as many points as possible were required for the selected ENDT tasks. As at least 30 points per contamination degree within a selected contamination scenario were required according to the model hypothesis, for the aspired POD demonstration, we primarily relied on the ENDT data obtained for the smooth coupon samples. Unlike with the more complex realistic and real CFRP specimens, the fact that numerous coupon samples were available allowed the inspection specialists to complement the ENDT datasets for which a POD calculation was performed, with a wide score of datasets obtained using LIBS, AWT, E-nose, EMI, and NLUS.

As this section aims to provide a vivid and demonstrative presentation of our POD approach, we focused on only one characteristic feature per ENDT technique. This technique-specific feature was selected to be "differentiating" for the respective contamination scenario. By this, we mean that the contemplated ENDT testing procedure was selected based on this characteristic feature so that not only could the contamination be detected compared to a clean reference specimen, but also different contamination levels could be distinguished. For this purpose, a feature value could be a measured amplitude or velocity or a value deduced from measured quantities using a model. Moreover, the correlation between this assessed feature and the contamination should be linearizable to respect the model hypothesis (see Fig. 5.50). The data input was requested as shown in Fig. 5.50 as an example contamination scenario. Hereby, we tested various scenarios and respective ENDT-based features.

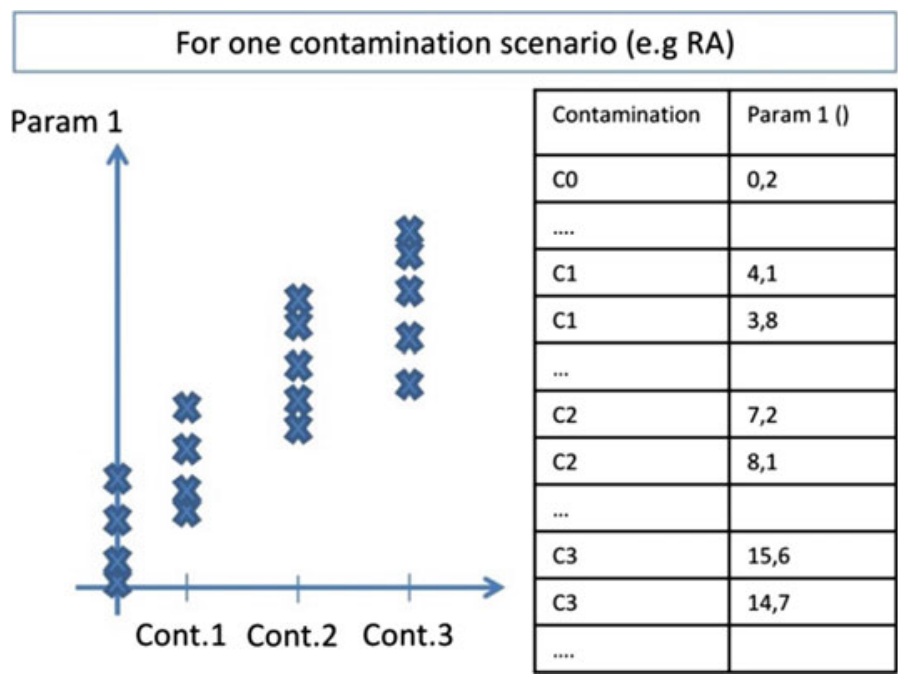

Fig. 5.50 Schematic description of the input data required for the exemplified POD approach. The contamination degrees Cont. 1, Cont. 2, and Cont. 3 were adjusted according to an accurate intentional contamination procedure and were characterized by laboratory-based analysis, while the ordinate values for Param 1 were obtained from the ENDT inspection 


\subsubsection{Choice of Contamination Values}

Here, we explain our cutting-edge approach and highlight the caution that guided how we proceeded during our proof of concept.

As, to our knowledge, the POD approach has never been used for ENDT techniques and the associated material-features, and as we aimed to introduce a quantitative probability of detection for contaminants, we adapted the contamination values included here in a way that the POD-based model converges. Indeed, the model was established for defects that are conventionally monitored in aeronautical applications, such as cracks or delaminations, which are generally quantified referring to a length in millimeters, with relatively small values. Pragmatically and to avoid any numerical issues, we thus decided to adapt and scale the contamination-related values from the laboratory analysis and ENDT to remain in an expected range for the used numerical tool. We list the thus obtained values in Table 5.9, and we briefly outline our value implementation for each contamination scenario. Essentially, we used a consistent procedure for all the contamination scenarios which had been realized centrally and characterized by surface-sensitive XPS laboratory investigations in a standardized way. For the local implementations of contamination scenarios, we relied on the feature values that were individually measured in laboratories. Furthermore, in the case of double-digit feature values, we scaled them as described subsequently.

- The contamination deposited on the CFRP specimens following the P-FP fingerprint scenario within the coupon sample production user case was quantified using the atomic surface concentrations $[\mathrm{Na}]$ and $[\mathrm{Cl}]$ given in at.\% as measured by XPS. For our POD calculation, we arbitrarily selected the $[\mathrm{Na}]$ values since they were in the expected range for the input parameters of the POD software.

- For the RA contamination of the respective P-RA contamination scenario, the [Si] as obtained by XPS was also directly used in the POD calculation.

- For the R-DI contamination scenario, which was based on depositing de-icing fluid on CFRP specimens within the repair user case, we used the [K] measured

Table 5.9 Adapted and scaled values from laboratory analyses for distinct contamination levels in the different contamination scenarios intentionally applied to CFRP specimens. Further details are given in the text

\begin{tabular}{l|l|l|l|l|l}
\hline Contamination & Reference & Level 1 & Level 2 & Level 3 & Unit \\
\hline P-FP & 0.01 & 0.2 & 0.5 & 0.7 & at.\% of Na \\
\hline P-RA & 0.1 & 3.2 & 5.1 & 6.2 & at.\% of Si \\
\hline P-MO (surface assessment) & \multicolumn{4}{|l|}{ Measured locally and individually, values were not merged } \\
\hline P-MO (bonding assessment) & 0.01 & 0.3 & 0.75 & 0.98 & Rel. humidity \\
\hline R-DI & 0.1 & 6.4 & 10.9 & 12.0 & at.\% of K \\
\hline R-FC & 0.1 & 1 & 2 & 3 & dimensionless \\
\hline R-TD & 0.01 & 0.4 & 0.8 & 1.0 & ${ }^{\circ} \mathrm{C} / 10$ \\
\hline R-FP & 0.01 & 0.2 & 0.5 & 1 & $\%$ of Skydrol oil \\
\hline
\end{tabular}


by XPS since the obtained values were in a range that complied with a conventional defect.

- In the case of the P-MO scenario regarding moisture uptake, we proceeded in a slightly different way because the exposure of the CFRP specimens to humid environments was performed and characterized locally. We did not merge these values but maintained their individual qualities for all assessments of surface quality. In contrast, as the adhesively bonded specimens were prepared locally and then passed on for bonding quality assessment, we used the respective communicated mean values.

- In the case of the false curing R-FC scenario, we proceeded in a slightly different way. As outlined above, the applied procedures for producing a weak bond did not rely on any contamination quantification. The only quantified characteristic was the curing temperature of the device used to bond the composite skin. However, this temperature could not plausibly be directly used as a contamination value. Therefore, we decided to use arbitrary values (see Table 5.9) in a way that a higher value represents a stronger deviation from the qualified bonding process.

- In contrast to the FC scenario, we used the overheating temperature as a value related to the incident level in the case of the thermal degradation R-TD scenario because the excess temperature was applied directly, similar to a thermal "contamination" on the skin. As the increments $+40^{\circ} \mathrm{C},+80^{\circ} \mathrm{C}$, and $+100^{\circ} \mathrm{C}$ constituted too great dimensionless values for the model input parameters, we divided them by 10 to remain in a smaller number range.

- In the case of the R-FP scenario, which was based on applying a fingerprint with Skydrol oil, the set value for the percentage concentration of Skydrol in the fingerprinted liquid was used as a contamination value.

Finally, we would like to note that for all the contamination scenarios, the respective reference values were deliberately set as hypothetical values, and in short, are supposed to represent features of cleanly prepared CFRP specimens. We chose each value to be at least ten times inferior to the value of the lowest applied and investigated contamination level. To be specific, it cannot be equal to zero, neither in practice nor in the model that is supposed to correctly converge. We estimated this specimen state and the respectively measured abscissa values to be a good material-related approach to provide the noise level for each ENDT technique.

\subsubsection{Examples of the First POD Evaluations}

In this section, based on the abovementioned considerations, we present the first POD calculations performed for the selected ENDT techniques and contamination scenarios, for which a promising data basis was provided within the production and repair user cases detailed in Chaps. 3 and 4. Since this was a preliminary investigation, we did not anticipate that any robust conclusions could be drawn for any ENDT technique since the certified POD requirements were not accounted for during the 
data acquisition. Rather, and from the point of view of the POD methodology, the aim was to establish whether our model hypotheses stood the first test. The two most prominent and restrictive boundary conditions under question were, first, the monotone evolution of the chosen and evaluated feature (specific to each ENDT method) with an increase of contamination, and second, the presumption of a comparable scattering within the data for each contamination level. We may anticipate here that the subsequently presented results appear encouraging, offer interesting trends, and provide a compass for the upcoming establishment of procedures for estimating ENDT-related POD_-indeed just what we aimed to elucidate.

\subsubsection{Evaluating the AWT Surface Quality Assessment}

As a first example, we present our findings of the POD calculation for a feature obtained from the aerosol wetting test (AWT) data obtained by using the bonNDTinspect device for the RA contamination scenario within the production user case.

The first feature that we investigated was the average droplet diameter observed after temporarily depositing primary droplets from a water spray onto intentionally contaminated CFRP surfaces. The respective AWT results are presented in Table 5.9. In this case, the POD model hypotheses are respected because the data are linearly correlated with the various contamination levels and the scattering reduces with the increasing contamination. In the next step, we positioned the detection threshold to differentiate the findings of the minor contamination level " 1 " from those for the cleaned reference surface. We determined that the set of measured feature values obtained for level 1 partially overlap with the reference dataset. Specifically, one point exceeds this tentatively set threshold. This finding affects the POD for the AWT technique for this scenario, although the detection probability remains good and achieves $\boldsymbol{a} \mathbf{9 0 / 9 5}=\mathbf{3 . 0}$ at. $\%$ for Si atoms (Fig. 5.51).

From the technological point of view, we would like to highlight that we will always be able to prepare CFRP surfaces with RA deposits that are so low that their AWT droplet pattern can barely be differentiated from that of a reference sample (or a set of reference samples). Thus, the technologically relevant aspect here is to highlight the importance that those contamination levels that significantly affect the fracture toughness, as an example of design-relevant criterion, can be reliably detected. As shown in Chap. 2 for the RA-1 contamination scenario, depositing an amount of Si-containing RA that corresponds to 3.0 at.\% Si (as measured by XPS) is related to a loss of the average fracture toughness. In detail, $G_{I C}$ and $G_{I I C}$ values are reduced by almost $18 \%$ and $37 \%$ of the value respectively observed for joints prepared with CFRP adherends with surfaces treated according to a qualification process. The second AWT-based feature that we investigated was the wettability instead of the droplet diameter. Based on essentially the same raw dataset, the thus obtained results were slightly better, as can be inferred from Fig. 5.52. Indeed, the evaluated data points for the lowest investigated contamination level (level 1) were not in the range of the data for the reference surface state. When we used this feature, the detection threshold thus completely separated the reference data from the level 1 


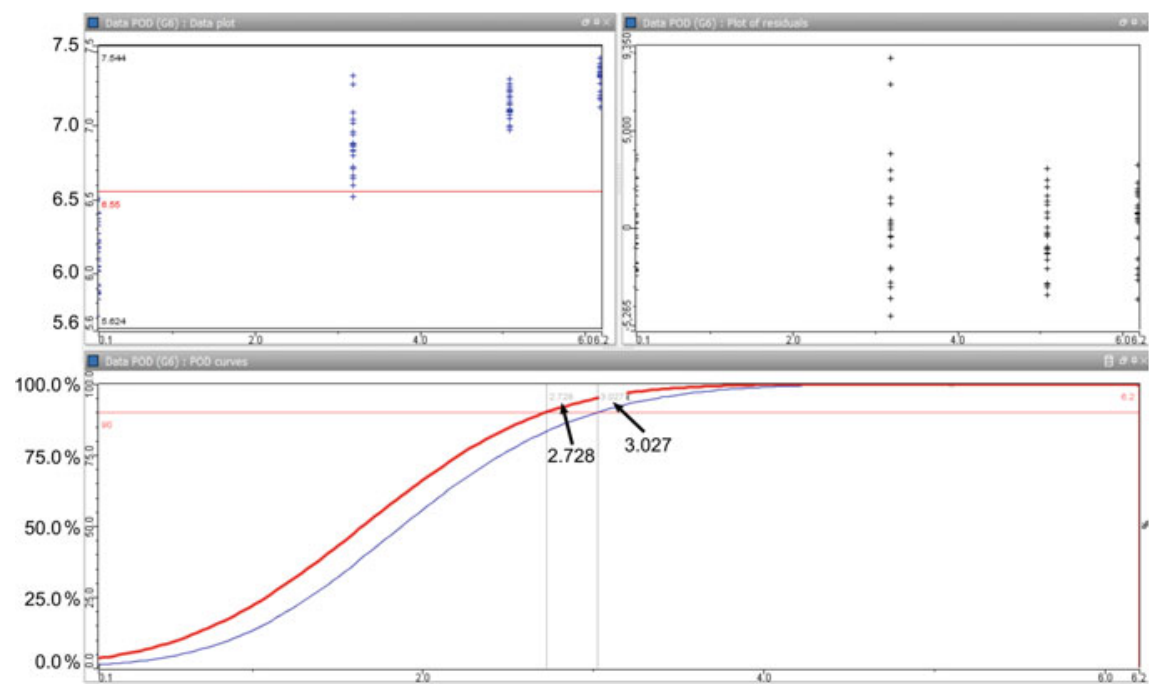

Fig. 5.51 POD curve for the AWT data obtained for the RA contamination scenario within the production user case when using the droplet diameter on the CFRP surface as the feature for evaluation

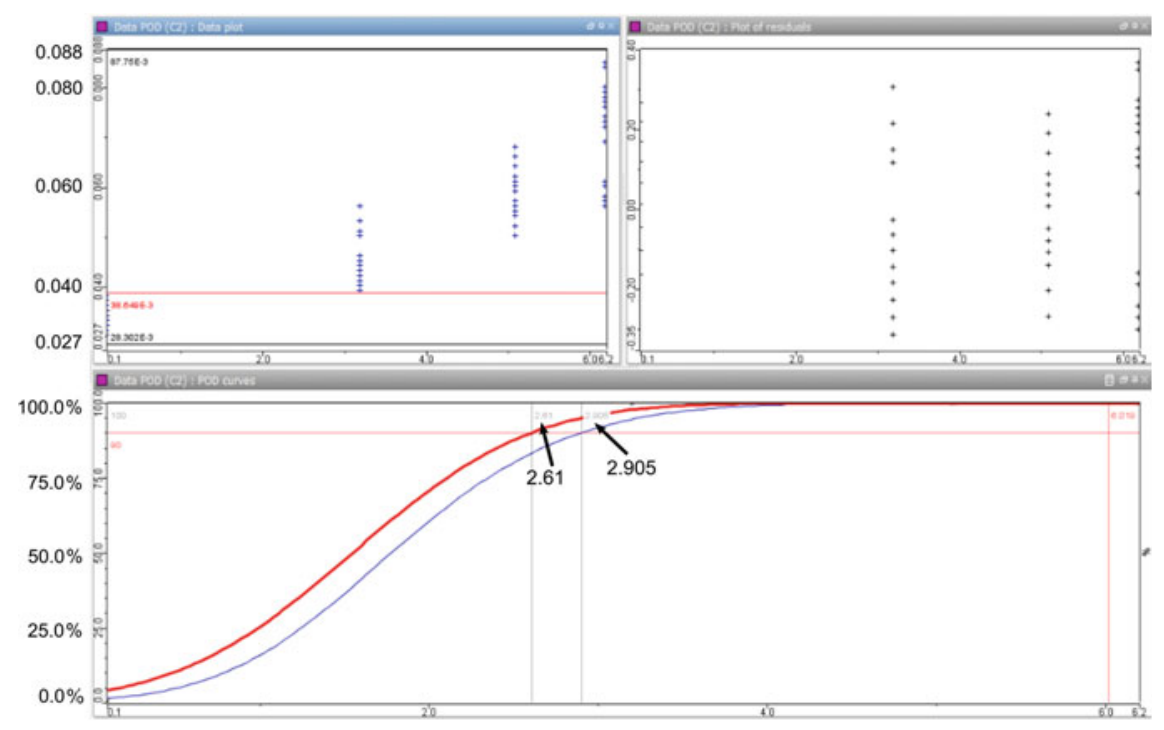

Fig. 5.52 POD curve for the AWT data applied for the RA contamination scenario within the production user case when using the wettability feature for evaluation 
data. As a consequence, the POD curve was more significant. The calculated $\boldsymbol{a 9 0 / 9 5}$ value corresponded to $\mathbf{2 . 9}$ at. \% of $\mathrm{Si}$, which is very similar to the outcome of applying the droplet diameter feature.

As a conclusion of these data post-processing options for the AWT findings, our POD investigations have shown that in the case of the RA contamination scenario, the performance of the testing procedure depends on the selection of the feature to be evaluated. We found a better detection performance when using the wettability feature compared to the droplet diameter feature. As a next step, from the data postprocessing point of view, we suggest performing a POD calculation by coupling the two approaches to determine whether the performance can be extended even further. From the test setup point of view, the area density of the applied aerosol droplets could be further adapted to be optimal for this scenario. We would like to highlight that at the end of the day, the multi-dimensional optimization of several parameters would be desirable, which would require extended modeling resources.

\subsubsection{Evaluating the FTIR Assessment in the Contamination Scenarios}

We performed POD calculations for several infrared spectroscopic (FTIR technique) datasets that had been performed for various contamination scenarios in both the production and repair user cases, namely moisture uptake in CFRP adherends, the deposition of de-icing fluid, and the thermal degradation of specimens. The respectively obtained results are presented as plots shown in Figs. 5.53, 5.54, and 5.55. For

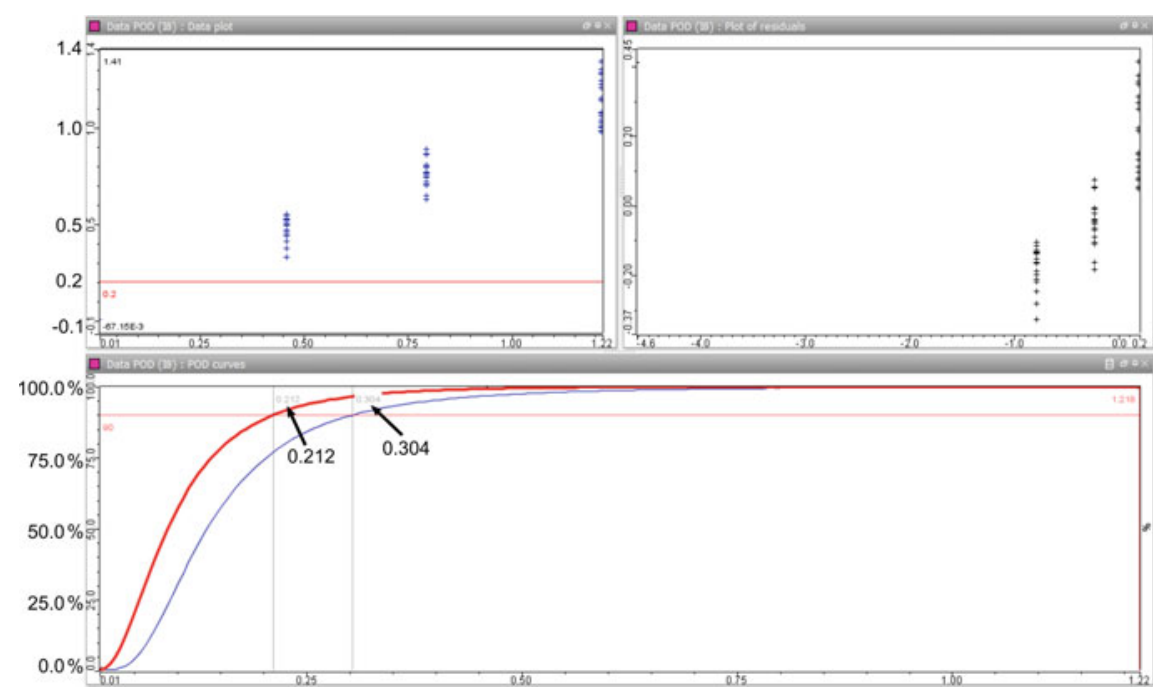

Fig. 5.53 POD curve for the FTIR datasets in the case of the MO contamination scenario within the production user case 


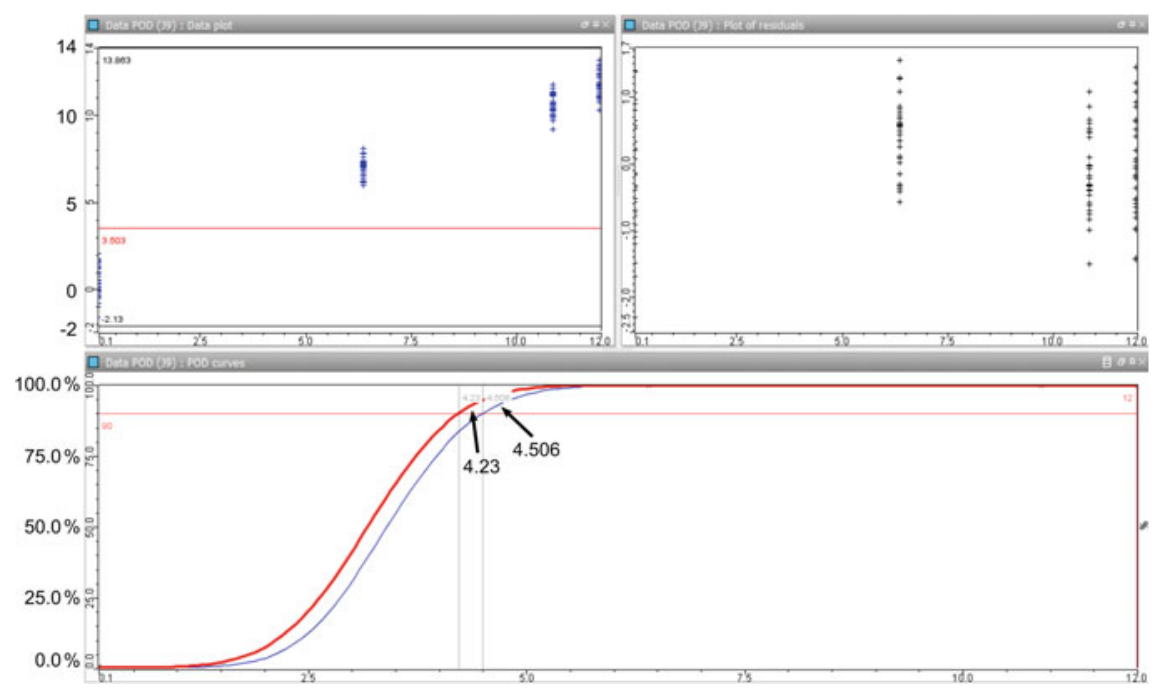

Fig. 5.54 POD curve for the FTIR datasets in the case of the DI fluid contamination scenario within the repair user case

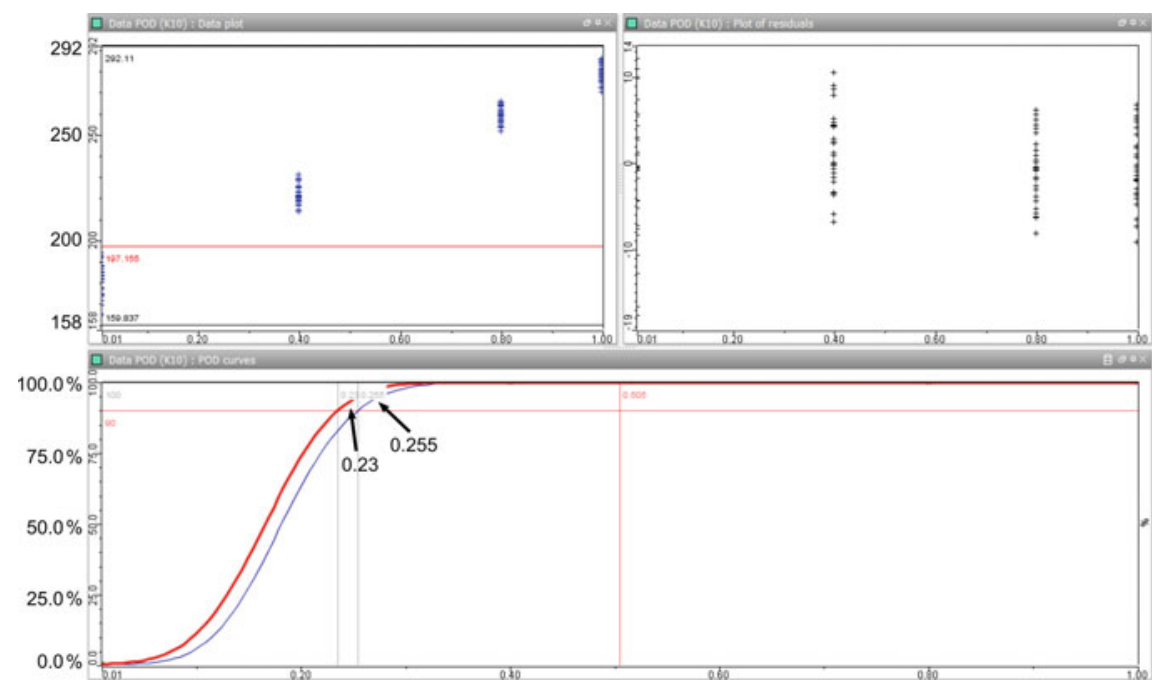

Fig. 5.55 POD curve for the FTIR datasets in the case of the TD scenario within the repair user case

all the scenarios, the data are well suited for POD calculation; only the scattering in the moisture scenario is slightly diverging. Otherwise, the detection threshold can be easily placed to separate the reference values from the contamination values. With 
no overlap between the reference and the contamination data, the POD performances are good. The values are presented in the following:

- In the case of the MO scenario, $a 90 / 95=30.4 \%$ of moisture uptake.

- In the case of the DI scenario, $a 90 / 95=4.5$ at. $\%$ of $\mathrm{K}$. This is good because the value is below the first contamination degree ( 6.4 at.\% of $\mathrm{K}$ ).

- In the case of the TD scenario, $a 90 / 95=25.5^{\circ} \mathrm{C}$ of overheating. This performance is good as the value is below the lowest contamination degree, which is equal to $40{ }^{\circ} \mathrm{C}$ of overheating.

\subsubsection{Evaluating LIBS Procedures for Surface Quality Assessment}

For the LIBS technique, we performed in-depth POD investigations and here present the findings related to the aspect of assessing and increasing the robustness and reliability of the technological procedure. We highlight the LIBS findings here because numerous datasets, each with a considerable number of data points, were available or were made available upon request. This was supported firstly by the fact that LIBS is a spectroscopic, and thus multimodal, technique that provides several quantifiable features and secondly by the sub-millimeter width of the spot-like CFRP surface area assessed with a single LIBS shot. We performed these investigations for the RA contamination scenario of the production user case for CFRP surfaces. For LIBS, the actual testing procedure during data acquisition includes a high number of measurements across surface regions that are small compared to, e.g., the lateral dimension of the intentionally applied contaminations. This means that during the data evaluation, an average calculation within a (grouped) set of data points may be performed to compensate for the variability among the locally acquired data.

Based on these considerations, we evaluated in more detail how different approaches to data processing affect the quality of the procedure in assessing qualityrelevant material surface features. In other words, our QA approach can be outlined as follows: First, the user operates the LIBS device to perform several measurements at various spots in a given area. During the processing of the thus achieved spectroscopic datasets, a spectroscopic feature is chosen-most often an intensity ratio of two spectral signals with one representing the RA deposit and one comprising the CFRP substrate. Then, an average is calculated for a group of feature values and is considered as a quality-relevant data processing result for the respective area. In the LIBS investigations described in Chap. 3, we experimentally established the number of involved LIBS shot measurements based on a confidence interval. Subsequently, we demonstrate how the procedure for refining this crucial criterion in the testing procedure may be enhanced by applying the POD tool. Specifically, for calculating the average values, we vary the size of the groups formed by the data points obtained from the raw data of the LIBS measurement that were acquired at neighboring surface positions.

Our starting point was a group size of one data point, meaning that we did not actually calculate the average and instead considered each single measurement point to be the result for the respective area that serves as the input data for the POD tool. 
The result of the POD calculation obtained for this boundary condition is presented in Fig. 5.55. In this case, 60 data points were used per contamination level (also including the reference surface state, which was also attributed to a contamination degree of 0.1). Clearly, these are raw data and no averages were calculated. The displayed data show that along with the increase of the contamination degree, there exists a considerable overlap between the reference data range and the data range for the contaminated samples. This finding hindered us from correctly positioning a detection threshold since either many points from contaminated samples would be below this threshold or some data points from non-contaminated surface positions would be above it. This uncertainty surrounding the POD result means that it is not satisfactory. The $a 90 / 95$ does not even appear on the graph (as presented in Fig. 5.55). These outcomes indicate, as already outlined in Chap. 3, that it is mandatory to perform average calculations during the data processing to increase the reliability of QA approaches based on the LIBS technique.

Therefore, we moved on to develop testing procedures based on calculating the average values from the raw data obtained from several LIBS shots. First, we calculated the averages over groups of ten points, thereby obtaining the outcomes of the POD calculation, as presented in Fig. 5.56. We worked with 120 points per contamination degree, including the reference. To some extent, we used some of the same data points as in the abovementioned first calculation. This means that we used 12 (averaged) values per contamination degree as input data for the POD calculation. Initially, this dataset appears somewhat inadequate, but it was worth further inspection, and indeed, the POD calculation offered a better curve than previously. It was

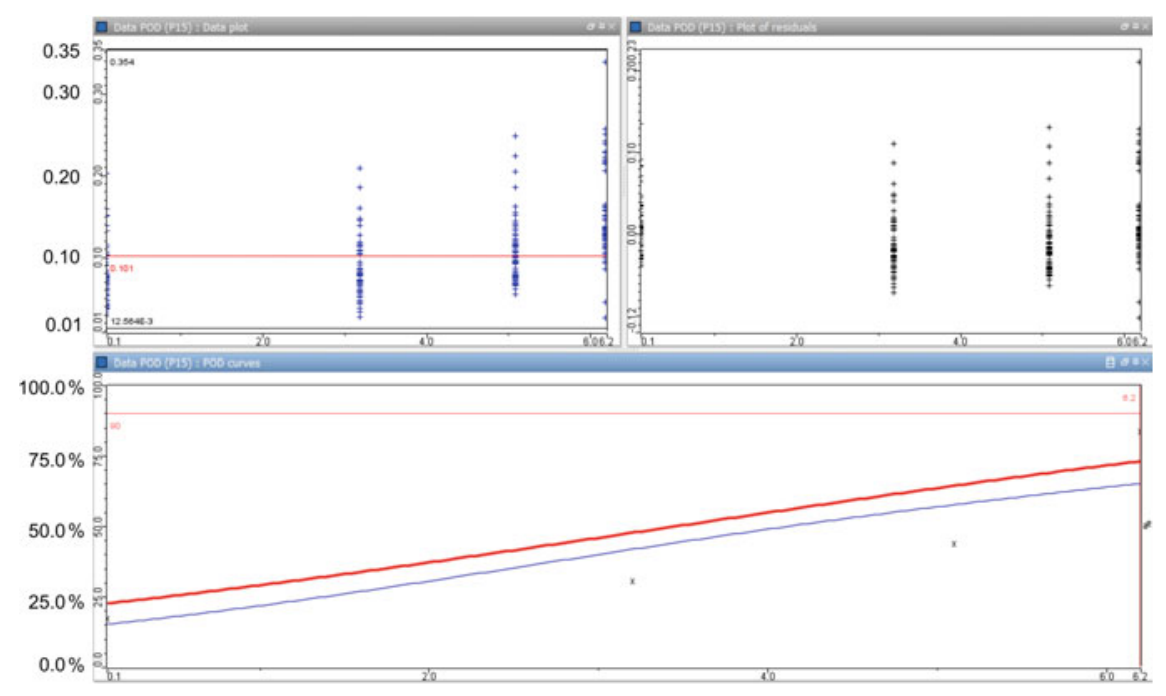

Fig. 5.56 POD curve for the LIBS datasets in the case of the RA contamination scenario within the production user case; the POD findings shown here were obtained by considering an evaluation procedure without averaging 


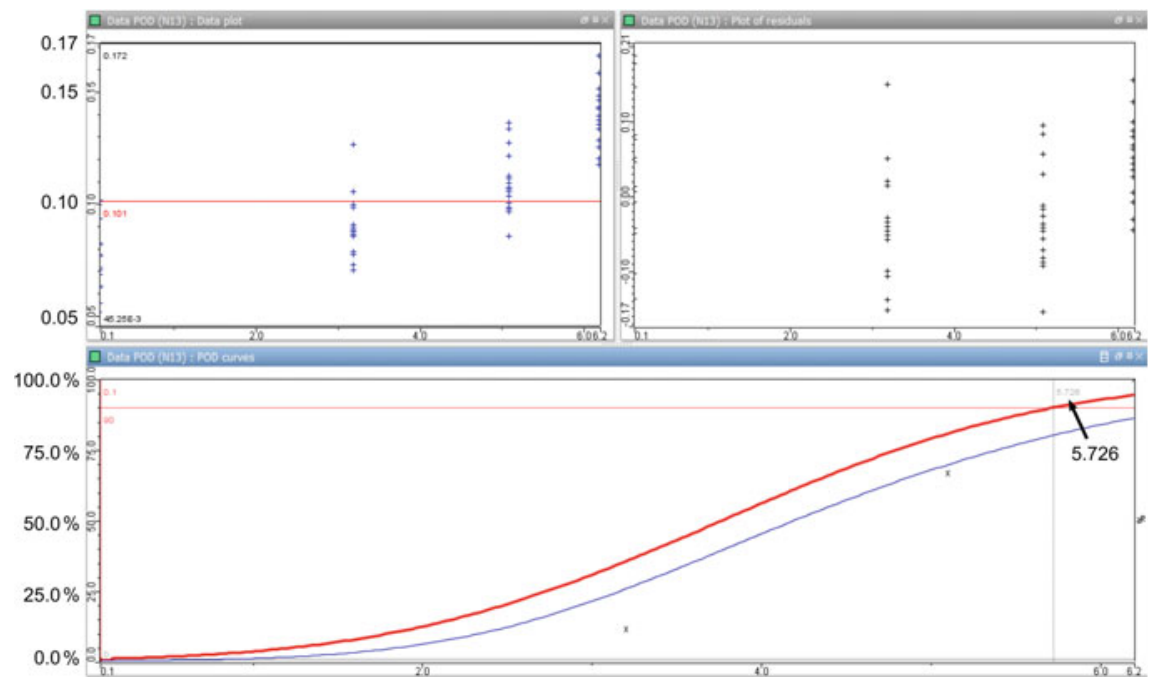

Fig. 5.57 POD curve for the LIBS datasets in the case of the RA contamination scenario within the production user case. The POD findings shown here were obtained considering an evaluation procedure involving an average measurement per group of ten data points

still not possible to perfectly differentiate data points for the reference specimen from the points for the first two contamination levels, but the third level was correctly determined based on the detection threshold. This enhanced approach thus led to a better probability of detection, even if the $a 90 / 95$ value was not yet obtained.

Hence, we had gained sufficient evidence to suggest another series of measurements that were performed specifically for our POD investigations. Within this advanced procedure, 36 measurement points were included from each surface region of the CFRP specimen, and a total of 20 regions were inspected for each contamination degree (including the reference). The POD calculation results presented in Fig. 5.57 are thus based on an input dataset comprising 20 data points per degree, with each point being an average of 36 measurements. The achieved results show a much better probability of detection. We did not find any overlap between the data ranges of the different contamination degrees, and we could identify and even reduce the detection threshold. As all data points from the contaminated surface regions were above this detection threshold, the final POD result was better. The $a \mathbf{9 0 / 9 5}$ was evaluated to be about 3.2 at.\% of $\mathbf{S i}$. As shown in Chap. 2 for the RA-1 contamination scenario, depositing an amount of Si-containing RA that corresponds to 3.2 at.\% $\mathrm{Si}$ (as measured by XPS) is related to a loss of the $G_{I C}$ and $G_{I I C}$ values by almost $18 \%$ and $37 \%$, respectively.

Briefly, in addition to this POD quantification, this in-depth study of surface QA processes based on the LIBS technique for the RA scenario has provided some interesting implications for establishing this testing procedure. For this contamination scenario, it emerged that doing averages over ten measurements for a total of 


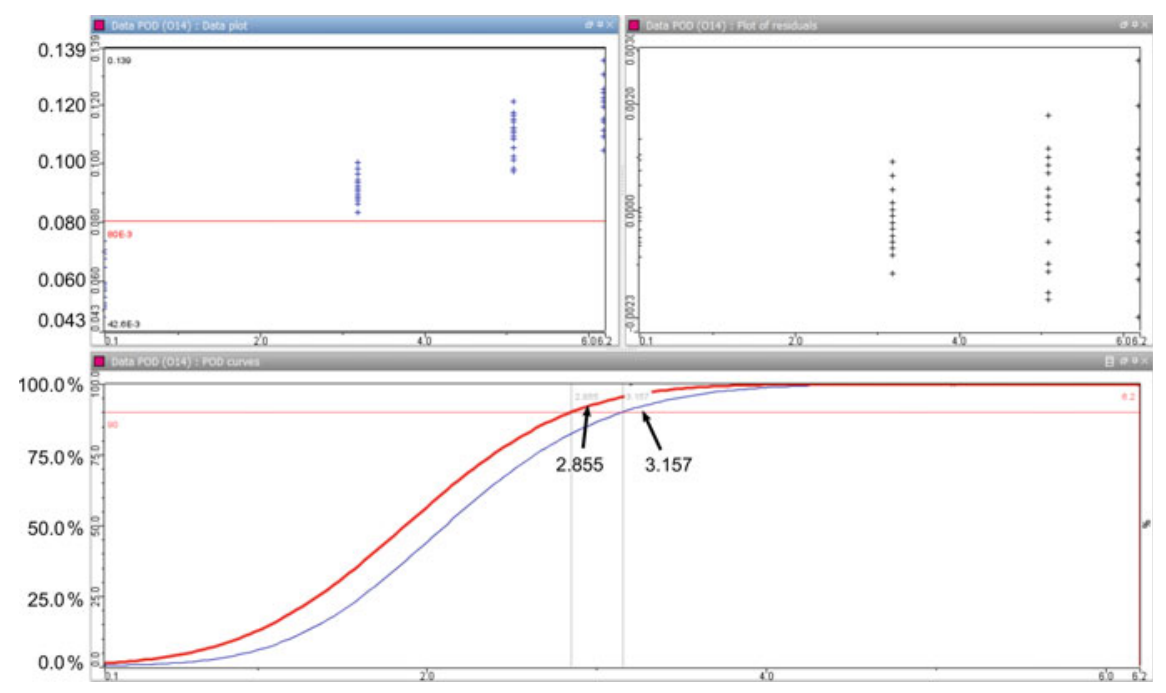

Fig. 5.58 POD curve for the LIBS datasets in the case of the RA contamination scenario within the production user case. The POD findings shown here were obtained considering an evaluation procedure involving an average measurement per group of 36 data points

120 LIBS shots is not sufficient to obtain a good POD. By averaging 36 measurements and performing 720 analytical shots, the POD improves to the point that it can even be quantified. This overall data acquisition and evaluation process can be maintained if the technique meets the detection requirements set for testing the design-relevant mechanical performance of adhesive joints manufactured based on the assessed CFRP adherends. We expect that the detection performance could be further improved by evaluating more and/or larger average groups, as might be revealed by another POD investigation. Clearly, establishing the optimum lateral distance between neighboring LIBS spots should also consider the total measurement time required per CFRP surface area. In this respect, many of the presently applied aeronautical user cases that require a significant number of manually performed operations offer a sufficient time "buffer" for LIBS-based surface quality monitoring (Fig. 5.58).

\subsubsection{Evaluating NLUS for Bonding Quality Assessment}

As an example of ENDT techniques for bonding quality assessment, we performed POD investigations based on datasets obtained using nonlinear ultrasound (NLUS) for five different contamination scenarios from the repair and production user cases. The respectively obtained results for the P-FP, P-MO, P-RA, R-DI, and R-FC scenarios are presented in this section in, Fig. 5.59, Fig. 5.60, Fig. 5.61, Fig. 5.62, and Fig. 5.63, respectively. For this ENDT technique, the hit/miss approach was used. 


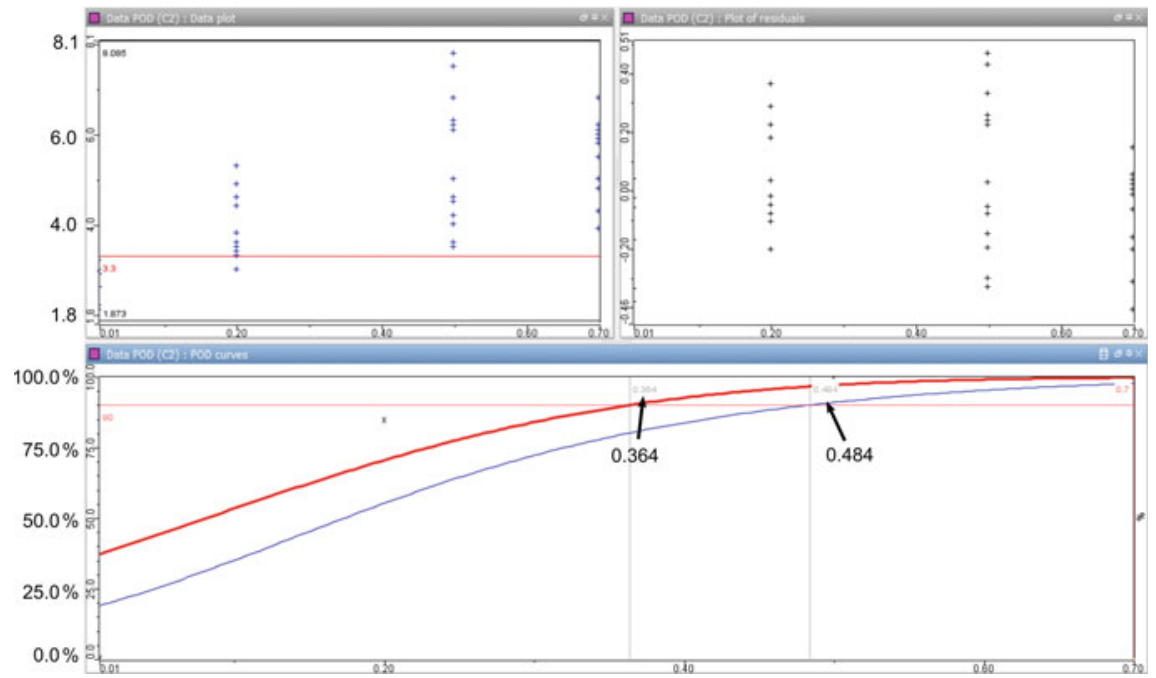

Fig. 5.59 POD curve for the NLUS datasets from the FP contamination scenario, applying a hit/miss model approach
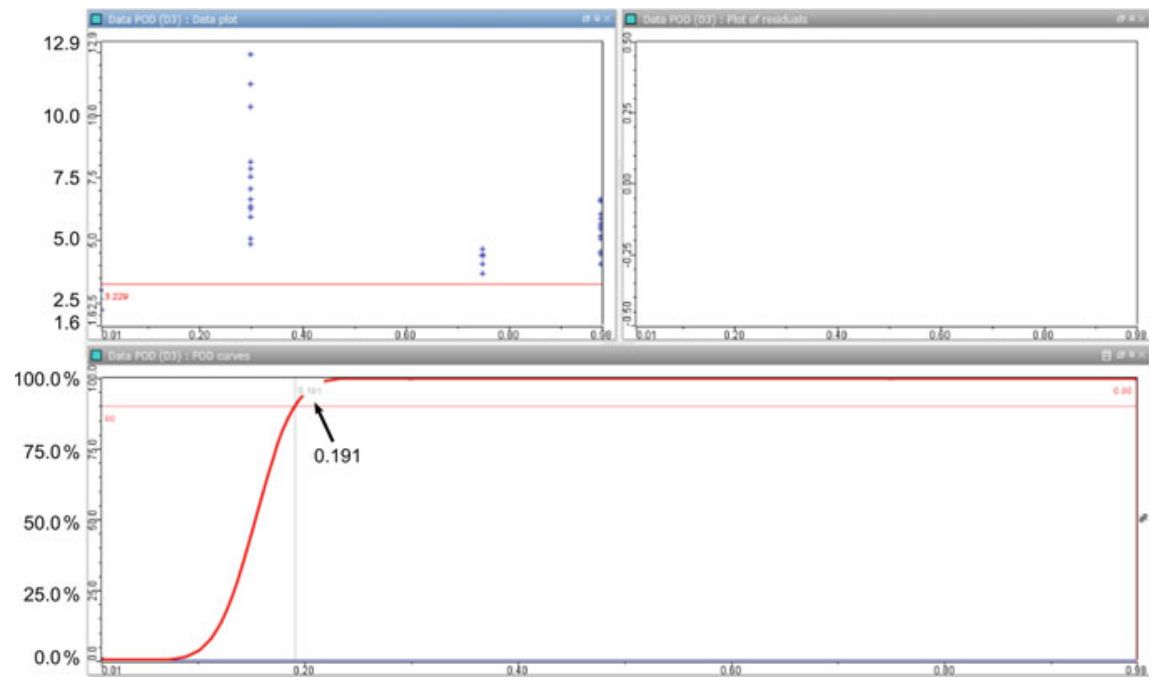

Fig. 5.60 POD curve for the NLUS datasets from the MO contamination scenario, applying a hit/miss model approach 


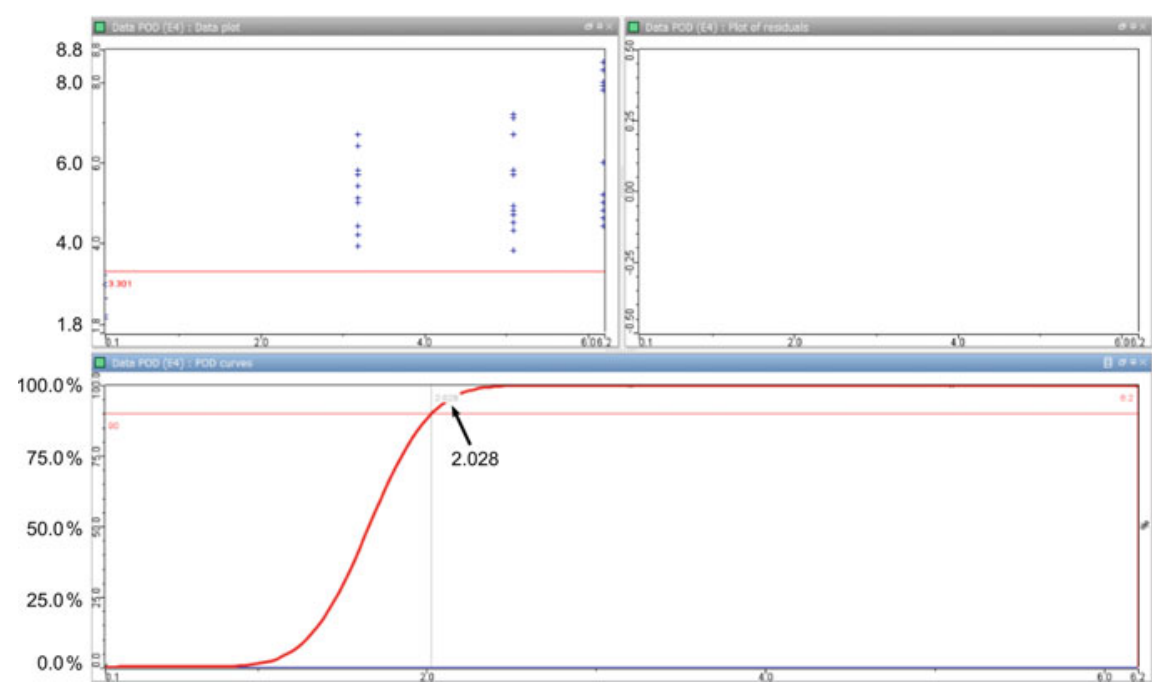

Fig. 5.61 POD curve for the NLUS datasets from the RA contamination scenario, applying a hit/miss model approach

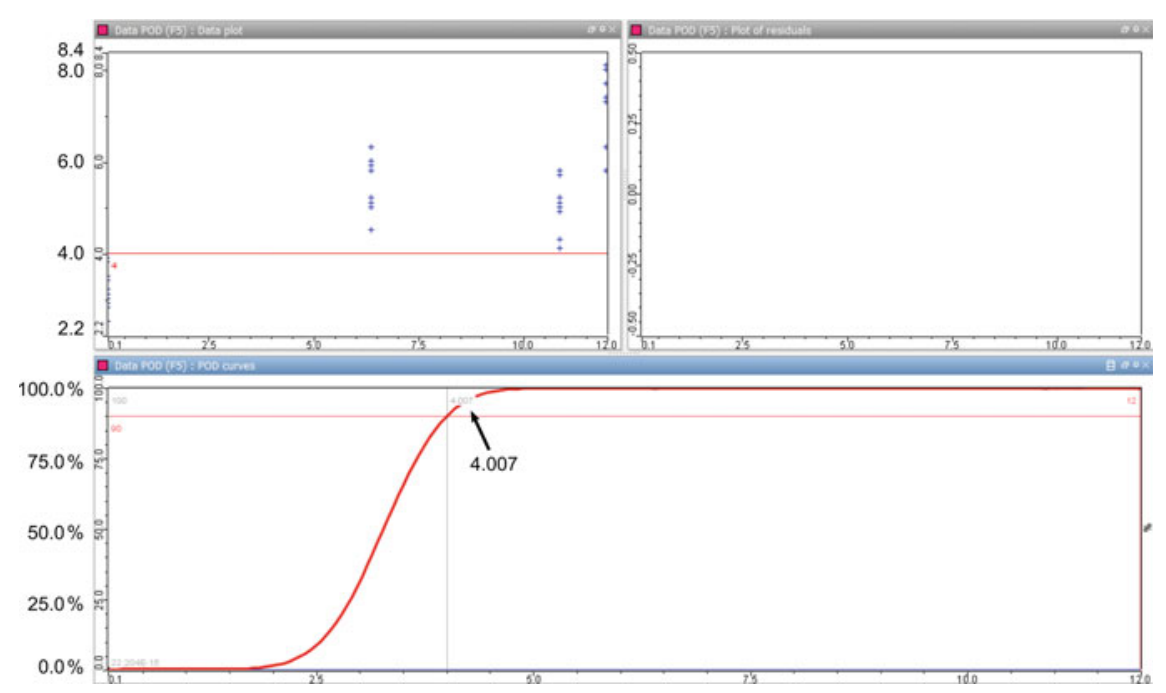

Fig. 5.62 POD curve for applying NLUS as an ENDT technique for the DI contamination scenario, applying a hit/miss model approach

After examining the NLUS datasets for each scenario, this approach was motivated on the one hand by the finding that the correlation between the feature signal amplitude and the applied contamination level was never monotonous or linear. However, the values obtained for the adhesive joints prepared from intentionally contaminated 


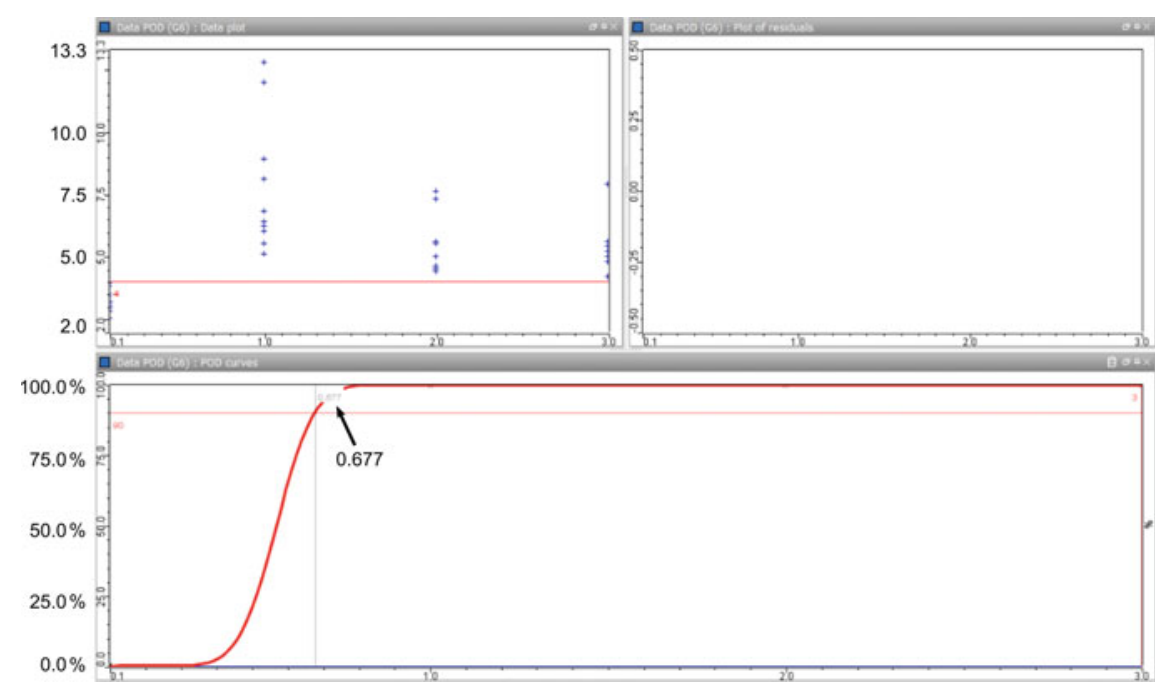

Fig. 5.63 POD curve for applying NLUS as an ENDT technique for the FC contamination scenario, applying a hit/miss model and tentatively excluding an assumed outlying data point

adherends were well separated from those found for the reference specimens, and this finding enables an NLUS expert to make a diagnosis since, based on their experience, such a signal pattern is typical for ultrasound diagnostics. Effectively, when the signal amplitude exceeds a given threshold (called a "gate" in the case of conventional ultrasound), then a defect is indicated. This behavior is thus well suited to a procedure based on the hit/miss model approach.

The results are detailed in the captions of the respective figures and are summarized comprehensively below. We would like to remind the reader here that applying the hit/miss model requires 60 data points per contamination degree. As during our orienting approach we used only ten data points, we would like to highlight that some care shall be taken before drawing in-depth conclusions.

- For the P-FP scenario, the data points for contamination level 1 were in the range of the values obtained for the reference specimen state. For a given contamination level, this decreased the probability of detection. The $a 90 / 95$ is about 0.48 at. $\%$ of $\mathrm{Na}$ (as measured by XPS), which roughly corresponds to contamination level 2. However, the probability of false detection is high. As shown in Chap. 2 for the P-FP-2 contamination scenario, depositing an amount of Na-containing fluid that corresponds to 0.5 at.\% Na (as measured by XPS) to a loss of the $G_{I C}$ and $G_{I I C}$ values by $8 \%$ and $61 \%$, respectively, as compared to the values observed for joints prepared with CFRP adherends with surfaces treated according to a qualification process.

- For the MO scenario, better discrimination between each contaminated sample and the reference specimen was obtained, leading to $a 90 / 95=19 \%$ of relative humidity. As shown in Chap. 2 for the MO-1 scenario, incorporating an amount 
of water that corresponds to $30 \%$ r.h. is not related to a loss of the $G_{I C}$ value but to a significant loss of the $G_{I I C}$ values by $45 \%$.

- Plausible data were also obtained for the RA scenario. The calculated value was $a 90 / 95=2.0$ at. $\%$ of Si. As shown in Chap. 2, this surface concentration is smaller than the one obtained and measured by XPS in case of the most exiguous contamination level investigated following the RA-1 contamination scenario. For this scenario, a loss of the $G_{I C}$ and $G_{I I C}$ values by almost $18 \%$ and $37 \%$, respectively, was found.

- For the R-DI scenario, the POD calculation also showed good performance. The estimated $a 90 / 95$ was about 4 at.\% of $\mathrm{K}$, which was low enough to be below contamination level 1 . This uncovers a technologically relevant and interesting potential since, as shown in Chap. 2 following the R-DI-1 contamination scenario, depositing an amount of $\mathrm{K}$-containing fluid that corresponds to 6 at.\% $\mathrm{K}$ (as measured by XPS) is related to a loss of the $G_{I C}$ and $G_{I I C}$ values by almost $30 \%$ and $56 \%$, respectively.

The NLUS data evaluation for the FC scenario required slightly more attention than the other scenarios. Indeed, in the case of the R-FC scenario, only one point from contamination level 3 was below the detection threshold that was placed above the reference data range. Since very few points were available, this point alone had a strong influence on the POD calculation as it had more weight. The $a 90 / 95$ is equal to 2.5 , meaning it lies between bond strength levels 2 and 3. If we consider this point an outlier and exclude it from the data range, with plausible justification, we may estimate what the POD would be without it or with more data (assuming additional points above the threshold). The result of such a consideration is given in Fig. 5.63. This leads to $a 90 / 95=0.677$, i.e., below level 1 , which is much better than when considering this assumed outlier to be significant. From a data-driven perspective, we thus conclude that it appears to be implausible to weigh and use this value unless an appropriate justification is given, e.g., through experimental replication or a physicsbased explication. In essence, we recommend providing a sufficiently substantial and consolidated set of measured data points for any technically relevant POD approach.

\subsection{The Results of the Full-Scale Demonstration: An Overall Synopsis of the Technology's Performance}

As a final summary of this chapter, we propose Tables 5.10 and 5.11 as a synthesis of the ENDT performance that was evaluated during the three-day full-scale demonstration during the ComBoNDT project [1]. We considered both production and repair user cases that were based on realistic and real CFRP parts, respectively. We highlight that our retrospective perception that the implementation of the full-scale demonstration with an aeronautic scope of application, of which we summarize the discrimination performances here, was largely paved by the coupon test phase described in Chaps. 2, 3, and 4 of this book. 
Table 5.10 Synthesis table of the performance demonstration for extended non-destructive testing (ENDT) tools and procedures that were advanced and applied during the ComBoNDT [1] project for CFRP specimens with flat (coupon samples) or complex (pilot and full-scale parts) shapes. The materials represent technologically relevant production or repair user cases for which different contamination scenarios had been identified, namely the exposure to moisture (MO), release agent (RA), fingerprint (FP), de-icing fluid (DI), false curing (FC), and thermal impact (TD) scenarios, as well as scenarios realized by simultaneously applying a combination of intentional contaminations. The ENDT techniques comprise Optically Stimulated Electron Emission (OSEE), Laser-induced breakdown spectroscopy (LIBS), two differently implemented approaches based on electronic noses (e-nose), laser vibrometry, Laser-Induced Fluorescence (LIF), an aerosol wetting test (AWT), Fourier transform infrared spectroscopy (FTIR), electromechanical impedance (EMI), magnetostrictive sensors (MGSS), nonlinear ultrasound (NLUS), and laser shock adhesion testing (LASAT). Further composite characterization was performed by ultrasound (US), micro-computed tomography $(\mu \mathrm{CT})$, and mechanical tests focusing on fracture toughness

\begin{tabular}{|c|c|c|c|c|c|c|c|c|}
\hline \multirow[t]{3}{*}{ ENDT } & \multicolumn{6}{|c|}{ COUPONS } & \multicolumn{2}{|c|}{ PILOT \& FULL SCALE } \\
\hline & \multicolumn{3}{|c|}{ PRODUCTION } & \multicolumn{3}{|c|}{ REPAIR } & \multirow{2}{*}{$\begin{array}{c}\text { PRODUCTION } \\
\text { RA+FP }\end{array}$} & \multirow{2}{*}{$\begin{array}{c}\text { REPAIR } \\
\text { R-DI+FP }\end{array}$} \\
\hline & MO & RA & FP & DI & FP / FC & TD & & \\
\hline OSEE & $\mathrm{V}$ & $\mathrm{V}$ & $\mathrm{V}$ & $\mathrm{V}$ & $\mathrm{V}$ & $\mathrm{X}$ & & \\
\hline LIBS & $\mathrm{X}$ & $\mathrm{V}+$ & $\mathrm{V}$ & $\mathrm{V}+$ & $\mathrm{V}+$ & $\mathrm{X}$ & $\mathrm{V}$ & \\
\hline E-nose & $\mathrm{V}$ & $\mathrm{V}$ & $\mathrm{V}$ & $\mathrm{V}$ & $\mathrm{V}$ & V & $\mathrm{V}+$ & $\mathrm{V}+$ \\
\hline Vibrometry & $\mathrm{V}$ & $\mathrm{X}$ & $\mathrm{X}$ & $\mathrm{X}$ & $\mathrm{X}$ & $\mathrm{X}$ & & $\mathrm{X}$ \\
\hline LIF & $\mathrm{X}$ & $\mathrm{X}$ & $\mathrm{X}$ & $\mathrm{X}$ & $\mathrm{X}$ & $\mathrm{V}_{220^{\circ} \mathrm{C}}$ & & $\mathrm{X}$ \\
\hline AWT & $\mathrm{V}+$ & $\mathrm{V}+$ & $\mathrm{V}$ & $\mathrm{V}$ & $\mathrm{X}$ & $\mathrm{V}$ & $\mathrm{V}$ & $\mathrm{V}+$ \\
\hline FTIR & $\mathrm{V}+$ & $\mathrm{X}$ & $\mathrm{X}$ & $\mathrm{V}+$ & $\mathrm{X}$ & $\mathrm{V}+$ & $\mathrm{X}$ & $\mathrm{N}^{\text {more data needed }}$ \\
\hline Mech. test & $\mathrm{V}+$ & $\mathrm{V}+$ & $\mathrm{V}+$ & $\mathrm{V}+$ & $\mathrm{V}+$ & $\mathrm{V}+$ & & \\
\hline US & $\mathrm{X}$ & $\mathrm{X}$ & $X$ & $\mathrm{X}$ & $\mathrm{V}+$ & $\mathrm{V}+$ & & \\
\hline$\mu \mathrm{CT}$ & V & V & V & V & $\mathrm{V}$ & V & & \\
\hline EMI & V & $X$ & $X$ & $\mathrm{X}$ & $\mathrm{V}$ & V & $\mathrm{N}$ & $\mathrm{X}$ \\
\hline MGSS & $\mathrm{X}$ & $\mathrm{X}$ & $\mathrm{V}$ & $\mathrm{X}$ & $\mathrm{V}$ & $\mathrm{V}$ & $\mathrm{X}$ & $\mathrm{V}^{\mathrm{FP}}$ \\
\hline NLUS & V & $\mathrm{V}+$ & $\mathrm{V}+$ & $\mathrm{V}+$ & $\mathrm{V}+$ & $\mathrm{V}+$ & $\mathrm{N}^{\text {more data needed }}$ & $\mathrm{N}^{\text {more data needed }}$ \\
\hline LASAT & $\mathrm{V}$ & $\mathrm{V}$ & $\mathrm{V}$ & $\mathrm{V}$ & $\mathrm{V}$ & $\mathrm{V}+$ & $\mathrm{V}$ & $\mathrm{V}$ \\
\hline CAPTION & $\mathrm{V}+\mathrm{De}$ & ion \& & rimin & $-V: d$ & ction $-\mathrm{X}: 1$ & detectic & / not conclusive- $\mathrm{N}$ : & $\mathrm{ial} /$ doubts remain \\
\hline
\end{tabular}

As we present the survey of our findings graphically, we would like to give some hints and details to ease the reading and to enhance the clarity of these summarizing presentations. Basically, we used a color code, marking the ENDT outcomes for the surface quality assessment in blue, the ENDT findings for the bonding quality assessment in green, and the respective laboratory-based analytical reference method findings for the bonded specimens in red. Then, for each cell, we use a code following the subsequently listed captions to estimate the performance of the inspection tools and procedures that we advanced and applied during the ComBoNDT project:

- "V+" shows that both the detection of and discrimination between different contamination levels or different contamination types in the case of multiple contaminations were achieved;

- "V" indicates that the detection of a contamination-based deviation from the reference specimen was managed;

- "X" denotes that a detection was not accomplished or was not conclusive;

- "N" means that only a part of the contamination scenario was captured or encompassed with some doubts remaining (see Table 5.10). 
Table 5.11 Synthesis table of the $a 90 / 95$ results obtained from the preliminary POD evaluations performed for the example ENDT techniques, the features assessed by data evaluation, and the contamination scenarios applied to CFRP adherends. The ENDT techniques examined here comprise the aerosol wetting test (AWT), Fourier transform infrared spectroscopy (FTIR), Laser-induced breakdown spectroscopy (LIBS), and nonlinear ultrasound (NLUS)

\begin{tabular}{l|l|l}
\hline ENDT technique & Contamination/evaluated feature & Calculated $a 90 / 95$ \\
\hline \multirow{2}{*}{ FWT } & P-RA/droplet diameter & 3.0 at. $\%$ of $\mathrm{Si}$ \\
\cline { 2 - 3 } & P-RA/wettability & 2.9 at. $\%$ of $\mathrm{Si}$ \\
\hline \multirow{2}{*}{ LIBS } & P-MO & $\begin{array}{l}\text { Moisture uptake upon exposure to } \\
30.4 \% \text { r.h. }\end{array}$ \\
\cline { 2 - 3 } & R-DI & 4.5 at. $\%$ of $\mathrm{K}$ \\
\cline { 2 - 3 } & R-TD & $25.5{ }^{\circ} \mathrm{C}$ of overheating \\
\hline & P-RA & 3.2 at. $\%$ of $\mathrm{Si}$ \\
\cline { 2 - 3 } & P-FP & 0.5 at. $\%$ of Na \\
\cline { 2 - 3 } & P-MO & $\begin{array}{l}\text { Moisture uptake upon exposure to } \\
19.1 \% \text { r.h. }\end{array}$ \\
\cline { 2 - 3 } & P-RA & 2.0 at. $\%$ of Si \\
\cline { 2 - 3 } & R-DI & 4.0 at. $\%$ of $\mathrm{K}$ \\
\cline { 2 - 3 } & R-FC & 2.542 \\
\hline
\end{tabular}

To provide quantitative indications of the ENDT performance, we evaluated example datasets obtained using different ENDT techniques and for different scenarios. For this purpose, we applied a POD approach that was reduced in complexity as compared to more formal and far more comprehensive technologically relevant procedures. The performances were quantified using the $a 90 / 95$ values. When available, the values are listed in Table 5.11. We highlight here again that this was only a first estimation since we did not perform a strict POD procedure to obtain these figures. We suggest that the next step should be to cross-check these values with the results from mechanically testing the adhesively bonded joints comprising intentionally contaminated adherends in order to correlate the POD with the expected decrease in the design-relevant mechanical material properties.

Furthermore, our simplified and preliminary POD investigations provided useful information. In some cases, a direct output of our POD approach was a refinement of ENDT testing procedures. For example, it was shown that evaluating the wettability feature was more promising than relying on the droplet diameter feature in the case of applying AWT for RA detection within the RA scenario. Similarly, the POD results also quantified the effect of the actually performed stepwise testing procedure on the probability of detection in the case of applying LIBS for this scenario. Indeed, better performances were obtained in this case by increasing the number of spot-wise measurements to an extent that was acceptable both from a performance and from a time-demand point of view.

In other cases, our POD approach showed that the assessed testing procedure was not yet sufficiently adapted as decreasing POD curves were obtained. That was the 
case, for example, when applying laser vibrometry for assessing specimens prepared following the MO scenario. However, our POD approach offered some clues on how to change the testing procedure to enhance the detection performance.

We conclude that the POD work introduced in this chapter represents the first step, and taking all the promising results into account, it would be interesting to push these investigations further as they offer much promise for the application of ENDT datasets obtained in the frame of a comprehensive real and adapted POD procedure.

\section{References}

1. ComBoNDT "Quality assurance concepts for adhesive bonding of aircraft composite structures by advanced NDT" (2015-2018) Project funded from the European Union's Horizon 2020 research and innovation programme under grant agreement No 636494

2. Berens AP (1989) NDE reliability data analysis. In: Nondestructive evaluation and quality control, 9th edn. ASM International, OH

3. PICASSO "imProved reliability inspeCtion pf Aeronautic structure through Simulation Supported POD" (2009-2012) Project funded under FP7-TRANSPORT and grant agreement No 234117

4. Pavlovic M, Zoëga A, Zanotelli C et al (2017) Investigations to introduce the probability of detection method for ultrasonic inspection of hollow axles at Deutsche Bahn. Procedia Struct Integr 4:79-86

5. Chung H-Y, Manuel L, Frank KH (2006) Optimal inspection scheduling of steel bridges using nondestructive testing techniques. J Bridge Eng 11:305-319. https://doi.org/10.1061/(ASC E)1084-0702(2006)11:3(305)

Open Access This chapter is licensed under the terms of the Creative Commons Attribution 4.0 International License (http://creativecommons.org/licenses/by/4.0/), which permits use, sharing, adaptation, distribution and reproduction in any medium or format, as long as you give appropriate credit to the original author(s) and the source, provide a link to the Creative Commons license and indicate if changes were made.

The images or other third party material in this chapter are included in the chapter's Creative Commons license, unless indicated otherwise in a credit line to the material. If material is not included in the chapter's Creative Commons license and your intended use is not permitted by statutory regulation or exceeds the permitted use, you will need to obtain permission directly from the copyright holder.

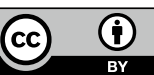

